\title{
Decarboxylative and Deaminative Alkylation of Difluoroenoxysilanes via Photoredox Catalysis: A General Method for Site-selective Synthesis of Difluoroalkylated Alkanes
}

Heng Song, ${ }^{\dagger}$ Ran Cheng, ${ }^{\ddagger}$ Qiao-Qiao Min, ${ }^{\ddagger}$ and Xingang Zhang ${ }^{\star, \dagger, \ddagger}$

'College of Chemistry, Henan Institute of Advanced Technology Zhengzhou University, Zhengzhou 450001, China ${ }^{*}$ Key Laboratory of Organofluorine Chemistry, Center for Excellence in Molecular Synthesis, Shanghai Institute of Organic Chemistry, University of Chinese Academy of Sciences, Chinese Academy of Sciences, 345 Lingling Lu, Shanghai 200032, China 


\section{Table of Contents}

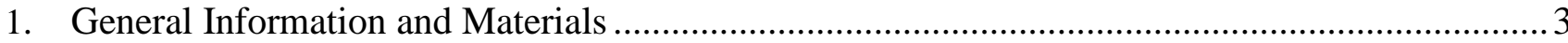

2. Optimization of Decarboxylative Alkylation of 2a with 1a ................................................. 3

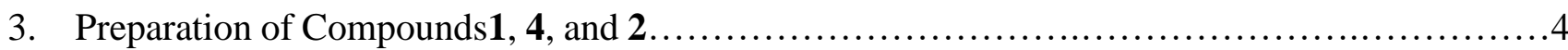

4. General Procedure for the Preparation of Compounds 3.............................. 9

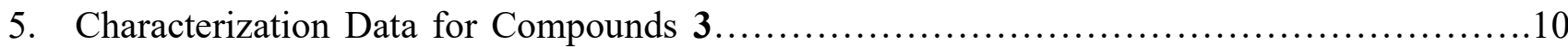

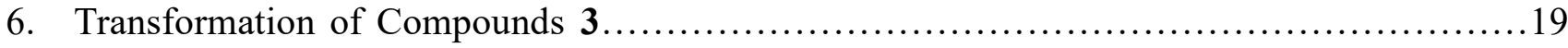

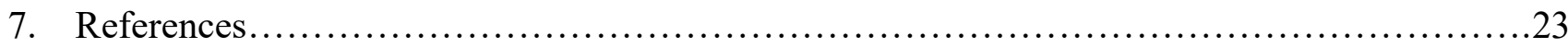

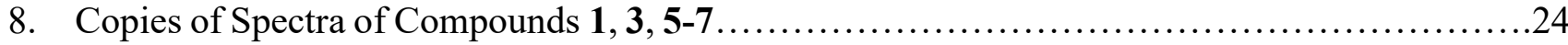




\section{General Information and Materials}

General Information: ${ }^{1} \mathrm{H}$ NMR and ${ }^{13} \mathrm{C}$ NMR spectra were recorded on a Bruker AM400, AM500 and Agilent 400 spectrometer. ${ }^{19} \mathrm{~F}$ NMR was recorded on a Bruker $\mathrm{AM} 400$ spectrometer $\left(\mathrm{CFCl}_{3}\right.$ as an external standard and low field is positive). Chemical shifts $(\delta)$ are reported in ppm, and coupling constants $(J)$ are in Hertz $(\mathrm{Hz})$. The following abbreviations were used to explain the multiplicities: $\mathrm{s}$ = singlet, $\mathrm{d}=$ doublet, $\mathrm{t}=$ triplet, $\mathrm{q}=$ quartet, $\mathrm{m}=$ multiplet, $\mathrm{br}=$ broad. NMR yield was determined by ${ }^{19} \mathrm{~F}$ NMR using fluorobenzene or trifluoromethylbenzene as an internal standard before working up the reaction.

Materials: All reagents were used as received from commercial sources. All reagents were weighed and handled under air. DMF, MeCN and DMSO were distilled under reduced pressure from $\mathrm{CaH}_{2} .1$,4Dioxane, diglyme, DME, $i \operatorname{Pr}_{2} \mathrm{O}$ and THF were distilled from sodium and benzophenone before use. All reagents were used from commercial suppliers or prepared using standard literature procedures.

\section{Optimization of Decarboxylative Alkylation of 2a with 1a}

To a $25 \mathrm{~mL}$ of glass Schlenk tube were added redox-ester $1 \mathbf{a}(187 \mathrm{mg}, 0.5 \mathrm{mmol}, 1.0$ equiv.) and photo catalyst $(0.005 \mathrm{mmol}, 1 \mathrm{~mol} \%)$. The mixture was evacuated and backfilled with argon for three times, difluoroenoxysilane $2 \mathbf{a}$ (427 mg, $1.5 \mathrm{mmol}, 3.0$ equiv.) and solvent (5 mL) were added. The reaction mixture was stirred at $60-65^{\circ} \mathrm{C}$ with blue LED $(12 \mathrm{w}, 460-465 \mathrm{~nm})$ as the heating source (the distance between the light source and Schlenk tube is $\sim 1.5 \mathrm{~cm})$ under irradiation of blue LED $(12 \mathrm{w}, 460-465$ $\mathrm{nm}$ ) for $24-48 \mathrm{~h}$. The yield was determined by ${ }^{19} \mathrm{~F}$ NMR using fluorobenzene as an internal standard. The reaction mixture was quenched by brine, extracted with ethyl acetate. The organic layer was dried over $\mathrm{Na}_{2} \mathrm{SO}_{4}$, filtered and concentrated. The residue was purified with silica gel chromatography to give product 3a. 


\section{Table S1. Optimization of Photoredox-Catalyzed Decarboxylative Alkylation of}

Difluoroenoxysilane 2a with Redox-Ester $1 a^{a}$

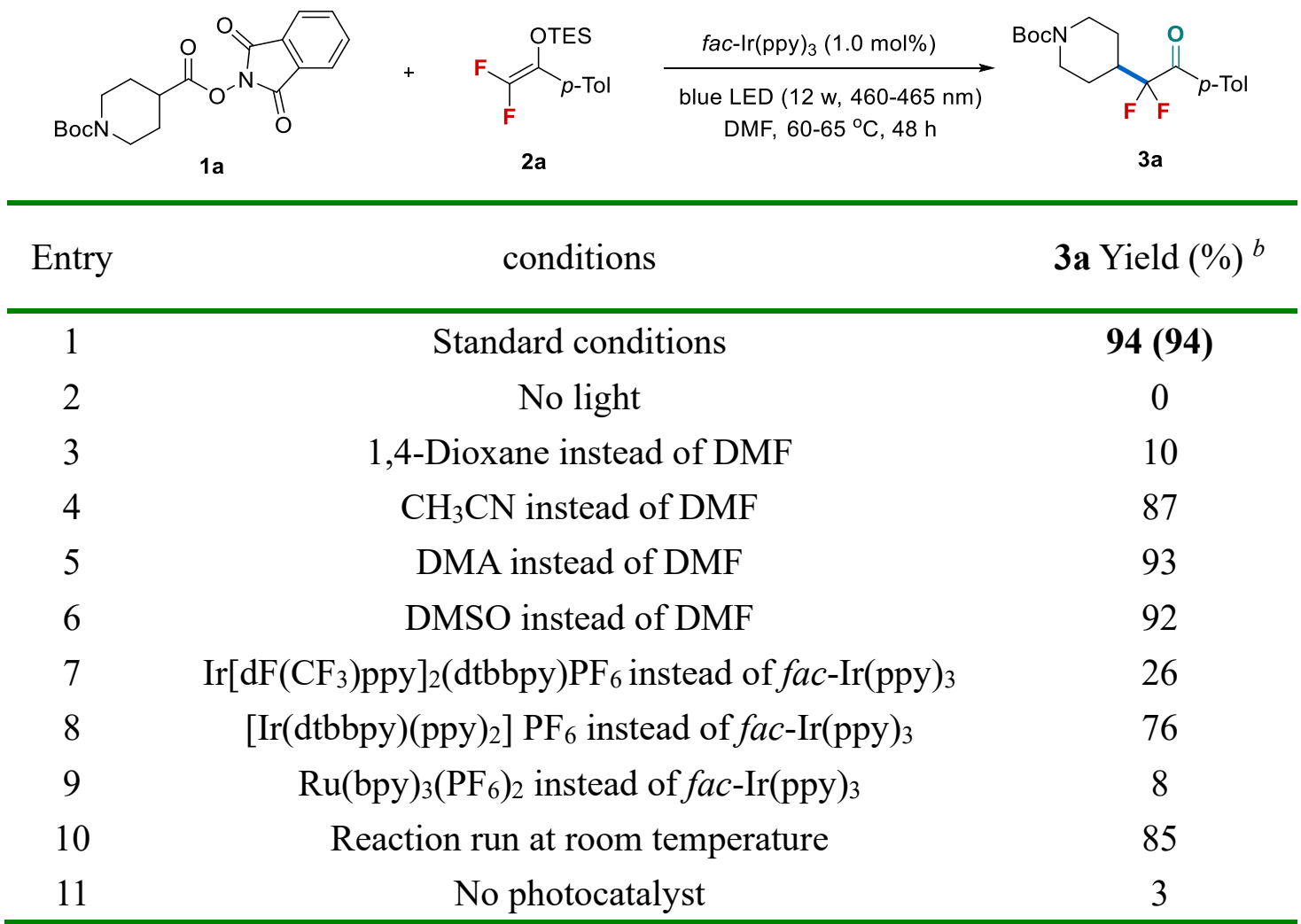

${ }^{a}$ Reaction conditions (unless otherwise specified): 1a ( $0.5 \mathrm{mmol}, 1.0$ equiv), $\mathbf{2 a}$ ( $1.5 \mathrm{mmol}, 3.0$ equiv), solvent (5 $\mathrm{mL}) .{ }^{b}$ Determined by ${ }^{19} \mathrm{~F}$ NMR using fluorobenzene as an internal standard.

\section{Preparation of Compounds 1, 4, and 2.}

\subsection{Preparation of Redox-Esters 1}

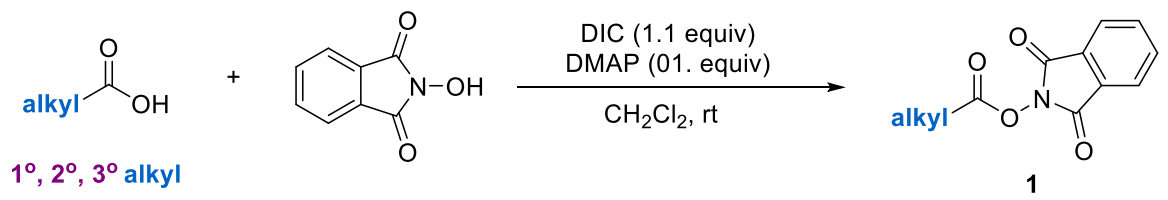

General Procedure: To a round-bottom flask were added alkyl carboxylic acid (1.0 equiv.), $\mathrm{N}$ hydroxy-phthalimide (1.0 equiv.), DMAP (0.1 equiv.), and dichloromethane (0.1 - $0.2 \mathrm{M})$. The mixture was stirred vigorously. DIC (1.1 equiv.) was then added dropwise via syringe, and the mixture was stirred at room temperature until the acid was consumed (determined by TLC). The mixture was filtered through celite and the filtrate was concentrated. The residue was purified with silica gel 
chromatography or recrystallization to give product $\mathbf{1}$.

Note: Redox- esters $1 \mathbf{a},{ }^{2} \mathbf{1 b},{ }^{2} \mathbf{1 c},{ }^{1} \mathbf{1 d},{ }^{4} \mathbf{1 f},{ }^{1} \mathbf{1 g}-\mathbf{j},{ }^{3}( \pm)$ trans-1o $,{ }^{2} \mathbf{1 p},{ }^{1}$ and $\mathbf{1 q},{ }^{4}$ were prepared according to the literature references.

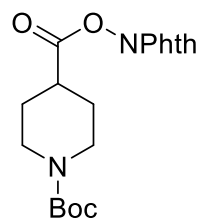

1a

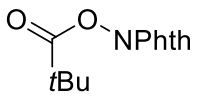

$1 \mathrm{~g}$

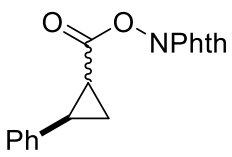

( \pm ) trans or $( \pm)$ cis-10

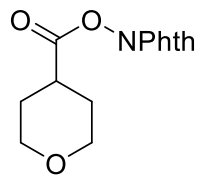

$1 \mathrm{~b}$

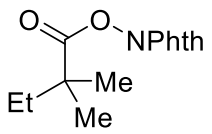

$1 \mathrm{~h}$<smiles>O=C(ONc1ccccc1)C1CCCCC1</smiles>

$1 c$

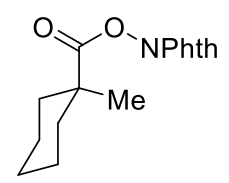

$1 \mathbf{i}$

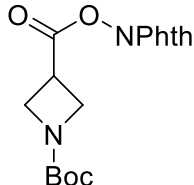

1d

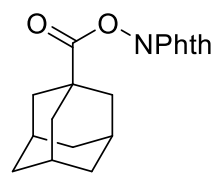

1j

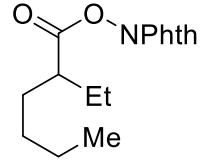

$1 e$<smiles>CCC(=O)ONc1ccccc1</smiles>

$1 \mathrm{k}$

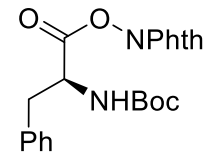

$1 f$

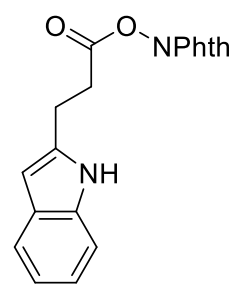

11<smiles>CC(C)C[C@H](NC(=O)[C@H](Cc1ccccc1)NC(=O)[O-])C(=O)O[PbH]c1ccccc1</smiles>

$1 \mathrm{~s}$

$\underbrace{O_{N P h t h}}_{\substack{E t \\ \mathrm{Me}}}$

1,3-Dioxoisoindolin-2-yl 2-ethylhexanoate (1e). The reaction was conducted on 10 mmol scale. The product 1e (2.36 g, $82 \%$ yield) as a yellow oil was purified by flash column chromatography (Hexane: Ethyl Acetate $=10: 1) .{ }^{1} \mathrm{H} \mathrm{NMR}\left(400 \mathrm{MHz}, \mathrm{CDCl}_{3}\right) \delta 7.88-7.85$ (m, 2H), $7.79-7.76(\mathrm{~m}, 2 \mathrm{H}), 2.69-2.58(\mathrm{~m}, 1 \mathrm{H}), 1.85-1.58(\mathrm{~m}, 4 \mathrm{H}), 1.51-1.31(\mathrm{~m}, 4 \mathrm{H}), 1.07$ (t, $J$ $=7.4 \mathrm{~Hz}, 3 \mathrm{H}), 0.94(\mathrm{t}, J=7.2 \mathrm{~Hz}, 3 \mathrm{H}) .{ }^{13} \mathrm{C} \mathrm{NMR}\left(100 \mathrm{MHz}, \mathrm{CDCl}_{3}\right) \delta 172.4,162.1,134.7,129.0$, $123.8,44.8,31.7,29.2,25.6,22.5,13.9,11.6$. MS (ESI): $\mathrm{m} / \mathrm{z}(\%)$ 163, $312.1[\mathrm{M}+\mathrm{Na}]^{+}$. HRMS (ESIquadrupole) m/z: [M+Na] $]^{+}$Calcd for $\mathrm{C}_{16} \mathrm{H}_{19} \mathrm{NO}_{4} \mathrm{Na} 312.1206$; Found 312.1203.

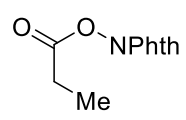

1,3-Dioxoisoindolin-2-yl propionate (1k). The reaction was conducted on 5 mmol scale. The product $1 \mathbf{k}\left(908 \mathrm{mg}, 83 \%\right.$ yield) as a white solid (m.p. $86-88{ }^{\circ} \mathrm{C}$ ) was purified by flash column chromatography (Hexane: Ethyl Acetate $=15: 1) .{ }^{1} \mathrm{H}$ NMR $\left(400 \mathrm{MHz}, \mathrm{CDCl}_{3}\right) \delta 7.89-$ 
$7.81(\mathrm{~m}, 2 \mathrm{H}), 7.80-7.69(\mathrm{~m}, 2 \mathrm{H}), 2.69$ (q, $J=7.6 \mathrm{~Hz}, 2 \mathrm{H}), 1.29(\mathrm{t}, J=7.6 \mathrm{~Hz}, 3 \mathrm{H}) .{ }^{13} \mathrm{C} \mathrm{NMR}(125$ $\left.\mathrm{MHz}, \mathrm{CDCl}_{3}\right) \delta 170.3,161.9,134.7,128.8,123.9,24.4,8.6 . \mathrm{MS}(\mathrm{ESI}): \mathrm{m} / \mathrm{z}(\%) 163(100), 242$ $[\mathrm{M}+\mathrm{Na}]^{+}$. HRMS (ESI- quadrupole) m/z: $[\mathrm{M}+\mathrm{Na}]^{+}$Calcd for $\mathrm{C}_{11} \mathrm{H}_{9} \mathrm{NO}_{4} \mathrm{Na} 242.0424$; Found 242.0421 .

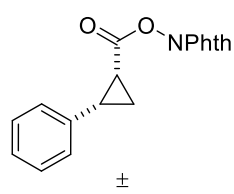

1,3-Dioxoisoindolin-2-yl 2-phenylcyclopropane-1-carboxylate $(( \pm)$-cis-10). The reaction was conducted on $2 \mathrm{mmol}$ scale. The product $( \pm)$-cis-10 (480 mg, yield $78 \%)$ as a white solid (m.p. $138-140^{\circ} \mathrm{C}$ ) was purified by flash column chromatography $($ Hexane: Ethyl Acetate $=5: 1) .{ }^{1} \mathrm{H}$ NMR $\left(400 \mathrm{MHz}, \mathrm{CDCl}_{3}\right) \delta 7.82-7.74(\mathrm{~m}, 2 \mathrm{H}), 7.73-7.66(\mathrm{~m}$, 2H), $7.41-7.29(\mathrm{~m}, 4 \mathrm{H}), 7.27-7.20(\mathrm{~m}, 1 \mathrm{H}), 2.97-2.88(\mathrm{~m}, 1 \mathrm{H}), 2.47-2.39(\mathrm{~m}, 1 \mathrm{H}), 1.85-1.78$ $(\mathrm{m}, 1 \mathrm{H}), 1.71-1.62(\mathrm{~m}, 1 \mathrm{H}) .{ }^{13} \mathrm{C} \mathrm{NMR}\left(125 \mathrm{MHz}, \mathrm{CDCl}_{3}\right) \delta 167.5,161.8,134.6,134.5,129.6,128.8$, 128.1, 127.3, 123.7, 28.0, 17.9, 13.8. MS (EI): m/z (\%) 145 (100), 307 [M] $]^{+}$HRMS (EI- TOF) m/z: $[\mathrm{M}]^{+}$Calcd for $\mathrm{C}_{18} \mathrm{H}_{13} \mathrm{NO}_{4}$ 307.0845; Found 307.0838.

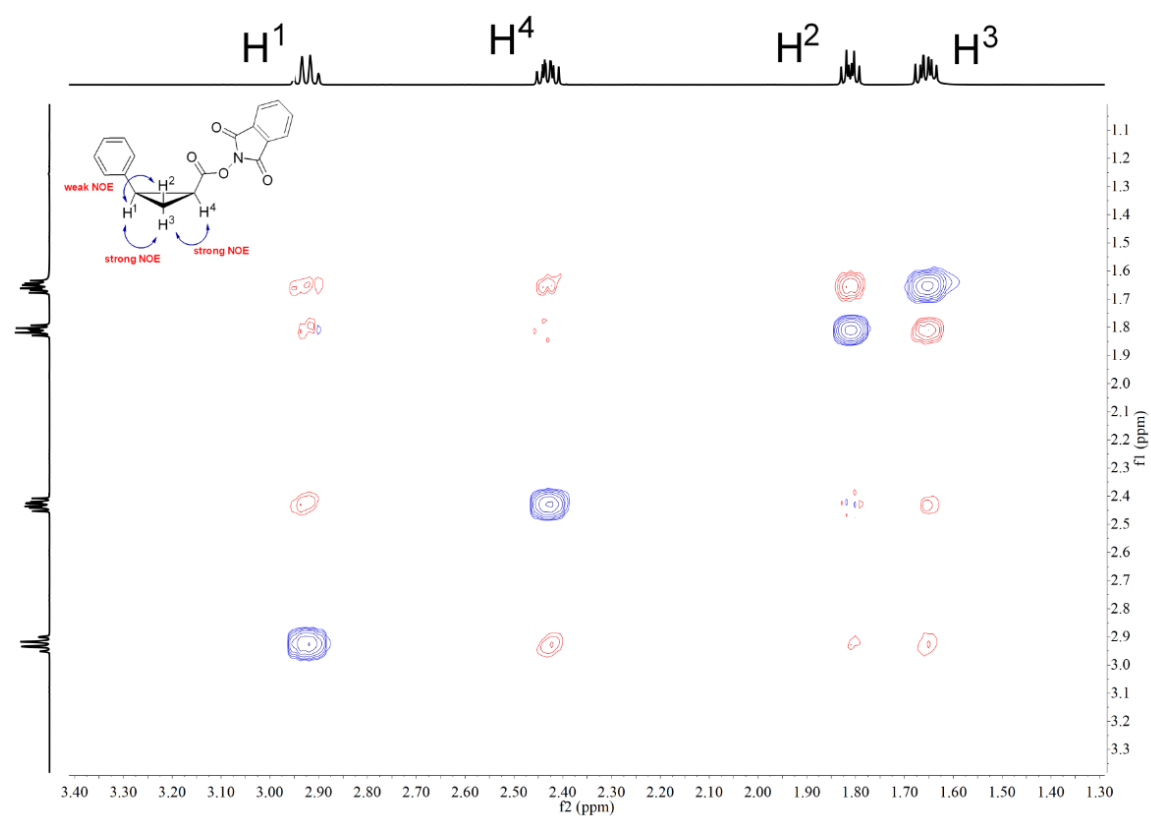

Figure S1. NOE Spectrum of Compound $1( \pm)-$ cis-10

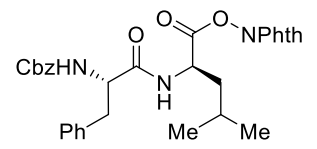

1,3-Dioxoisoindolin-2-yl ((benzyloxy)carbonyl)-L-phenylalanyl-D-

leucinate (1s). The reaction was conducted on $3 \mathrm{mmol}$ scale. The product $1 \mathrm{~s}$ (936 mg, yield $56 \%$ ) as a white solid (m.p. $158-160{ }^{\circ} \mathrm{C}$ ) was purified by flash column chromatography $($ Hexane: Ethyl Acetate $=5: 1) .{ }^{1} \mathrm{H}$ NMR $\left(400 \mathrm{MHz}, \mathrm{CDCl}_{3}\right) \delta 8.00-7.91(\mathrm{~m}, 2 \mathrm{H}), 7.89-7.82(\mathrm{~m}$, 
2H), $7.41-7.23(\mathrm{~m}, 10 \mathrm{H}), 6.88(\mathrm{br}, 1 \mathrm{H}), 5.90-5.69(\mathrm{~m}, 1 \mathrm{H}), 5.12(\mathrm{~s}, 2 \mathrm{H}), 5.08-4.98(\mathrm{~m}, 1 \mathrm{H}), 4.64$ (br, 1H), $3.25-3.06(\mathrm{~m}, 2 \mathrm{H}), 2.02-1.66(\mathrm{~m}, 3 \mathrm{H}), 1.07-0.94(\mathrm{~m}, 6 \mathrm{H}) .{ }^{13} \mathrm{C} \mathrm{NMR}\left(125 \mathrm{MHz}, \mathrm{CDCl}_{3}\right)$ $\delta 174.9,171.2,168.9,164.4,161.4,156.1,136.1,134.8,134.1,129.3,128.7,128.6,128.4,128.0$, $127.8,126.9,124.0,123.3,66.9,55.9,50.9,49.1,40.9,38.3,24.6,22.6,21.7 . \mathrm{MS}(\mathrm{ESI}): \mathrm{m} / \mathrm{z}(\%) 558$ $[\mathrm{M}+\mathrm{H}]^{+}$. HRMS (ESI- quadrupole) m/z: $[\mathrm{M}+\mathrm{H}]^{+}$Calcd for $\mathrm{C}_{31} \mathrm{H}_{32} \mathrm{~N}_{3} \mathrm{O}_{7}$ 558.2240; Found 558.2231.

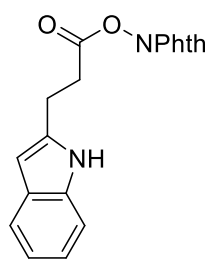

1,3-Dioxoisoindolin-2-yl 3-(1H-indol-3-yl)propanoate (11). The reaction was conducted on 5 mmol scale. The product 11 (1.42 g, yield $85 \%$ ) as a yellow solid (m.p. $155-157^{\circ} \mathrm{C}$ ) was purified by flash column chromatography (Hexane: Ethyl Acetate $=$ 10: 1). ${ }^{1} \mathrm{H}$ NMR $\left(400 \mathrm{MHz}, \mathrm{CDCl}_{3}\right) \delta 8.08(\mathrm{br}, 1 \mathrm{H}), 7.90-7.86(\mathrm{~m}, 2 \mathrm{H}), 7.80-7.76$ (m, 2H), $7.62(\mathrm{~d}, J=7.6 \mathrm{~Hz}, 1 \mathrm{H}), 7.38(\mathrm{~d}, J=8.0 \mathrm{~Hz}, 1 \mathrm{H}), 7.24-7.10(\mathrm{~m}, 3 \mathrm{H}), 3.26$ (t, $J=7.4 \mathrm{~Hz}$, 2H), $3.06(\mathrm{t}, J=7.6 \mathrm{~Hz}, 2 \mathrm{H}) .{ }^{13} \mathrm{C} \mathrm{NMR}\left(125 \mathrm{MHz}, \mathrm{CDCl}_{3}\right) \delta 169.3,162.0,136.2,134.7,128.9,126.9$, $123.9,122.2,121.9,119.5,118.4,113.6,111.2,31.8,20.4 . \mathrm{MS}(\mathrm{EI}): \mathrm{m} / \mathrm{z}$ (\%) 163 (100), $334[\mathrm{M}]^{+}$. HRMS (EI- TOF) m/z: [M] $]^{+}$Calcd for $\mathrm{C}_{19} \mathrm{H}_{14} \mathrm{~N}_{2} \mathrm{O}_{4}$ 334.0954; Found 334.0946.

\subsection{Preparation of Pyridinium Salts 4.}

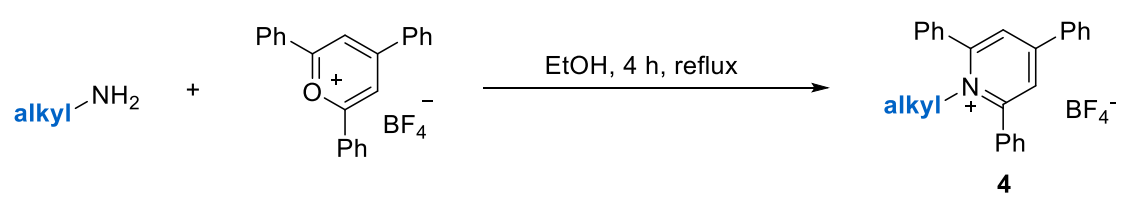

General Procedure: 2,4,6-Triphenylpyrylium tetrafluoroborate (1.0 equiv.) was suspended in absolute EtOH (1M). Subsequently, alkyl amine (1.2 equiv.) was added and the reaction mixture was refluxed at $90{ }^{\circ} \mathrm{C}$ for $4 \mathrm{~h}$. The reaction mixture was then cooled to room temperature and diluted with $\mathrm{Et}_{2} \mathrm{O}$. The resulting precipitate was filtered off, washed with $\mathrm{Et}_{2} \mathrm{O}$, and concentrated under vacuum at $50{ }^{\circ} \mathrm{C}$ to afford pure product 4 .

Pyridinium salts $\mathbf{4 a},{ }^{1}$ and $\mathbf{4} \mathbf{b}^{1}$ were prepared according to the literature references.

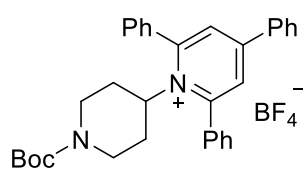

$4 a$

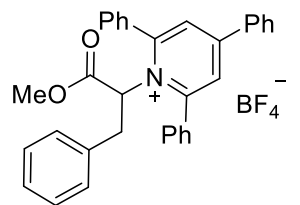

4b 


\subsection{Preparation of Difluoroenoxysilanes 2}

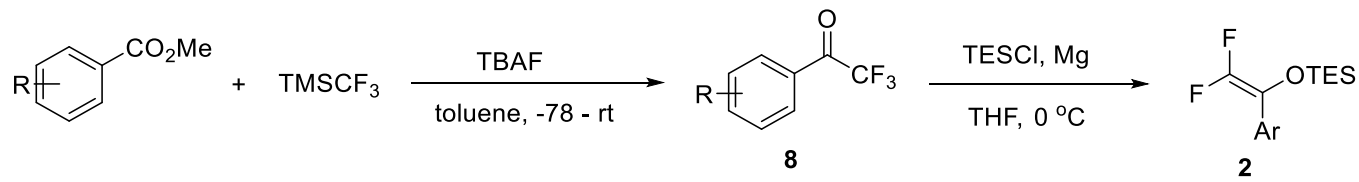

\section{Preparation of Ketones $8^{5}$}

Typical Procedure for the Preparation of 8a: To a solution of methyl 4-methyl-benzoate (15.0 g, 100 mmol, 1.0 equiv.) in toluene (200 mL) was added $\mathrm{TMSCF}_{3}(28.3 \mathrm{~g}, 200 \mathrm{mmol}, 2.0$ equiv.) at 25 ${ }^{\circ} \mathrm{C}$ under Argon. The reaction mixture was then cooled to $-78{ }^{\circ} \mathrm{C}$, TBAF $(10 \mathrm{~mL}, 1.0 \mathrm{M}$ in THF, 0.1 equiv.) was added dropwise. After stirring at $-78{ }^{\circ} \mathrm{C}$ for $0.5 \mathrm{~h}$, the reaction mixture was warmed to room temperature and stirred for $12 \mathrm{~h}$. Hydrochloric acid $(75 \mathrm{~mL}, 2.0 \mathrm{M}, 1.5$ equiv.) was then added and the resulting mixture stirred for $2 \mathrm{~h}$. The resulting suspension was quenched with saturated aqueous $\mathrm{NaHCO}_{3}$ and extracted with ethyl acetate. The combined organic layers were dried over $\mathrm{Na}_{2} \mathrm{SO}_{4}$, filtered, and concentrated. The residue was purified by flash column chromatography to give ketone 8a. Note: Compound 8a-c are known. ${ }^{5}$

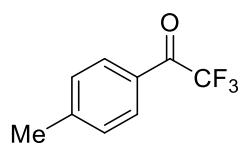

$8 \mathbf{a}$<smiles>O=C(c1ccccc1)C(F)(F)F</smiles>

$8 b$<smiles>CC(C)(C)c1ccc(C(=O)C(F)(F)F)cc1</smiles>

8c

\section{Preparation of Difluoroenoxysilanes $2^{5}$}

To a three-necked bottle was added $\mathrm{Mg}$ (5 g, $212 \mathrm{mmol}, 4.0$ equiv.). The mixture was evacuated and backfilled with argon for three times, then THF (250 mL) and TESCl (32 g, 212 mmol, 4.0 equiv.) were added. After the reaction mixture was cooled to $0{ }^{\circ} \mathrm{C}$, ketone 8 ( $10 \mathrm{~g}, 53 \mathrm{mmol}, 1.0$ equiv. $)$ was added dropwise and the resulting mixture was stirred for $40 \mathrm{~min}$. The resulting mixture was filtered with a pad of celite. The filtrate was concentrated, the residue was dissolved in hexane (500 $\mathrm{mL})$. The mixture was filtered again with a pad of celite. The filtrate was concentrated, and the residue was purified with silica gel chromatography to give difluoroenoxysilane 2. Note: Compounds 2a-c are known. ${ }^{5}$

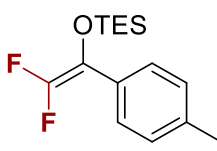

$2 a$

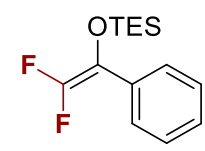

$2 b$

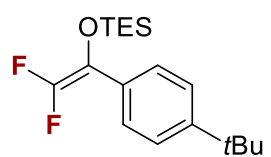

2c 


\section{General Procedure for the Preparation of Compounds 3.}

4.1. General Procedure for Decarboxylative Alkylation of Difluoroenoxysilanes 2 with RedoxEsters 1 (Procedure A)

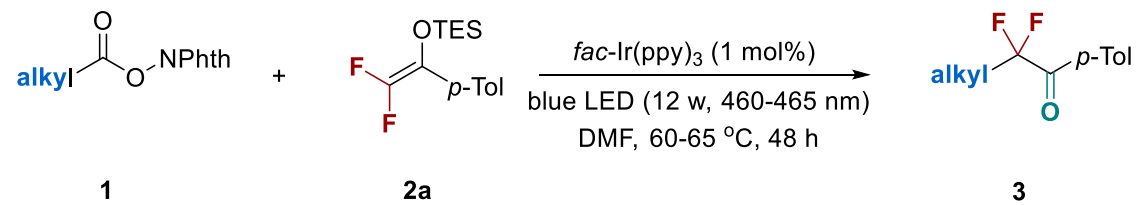

To a $25 \mathrm{~mL}$ of glass Schlenk tube were added redox-ester (1) (0.5 mmol, 1.0 equiv.) and $\mathrm{fac}$ - $\operatorname{Ir}(\mathrm{ppy})_{3}$ (3.27 mg, $0.005 \mathrm{mmol}, 1 \mathrm{~mol} \%$ ). The mixture was evacuated and backfilled with argon for three times, difluoroenoxysilane $2 \mathbf{a}(427 \mathrm{mg}, 1.5 \mathrm{mmol}, 3$ equiv.) and DMF (5 mL) were added. The reaction mixture was stirred at $60-65^{\circ} \mathrm{C}$ with blue LED $(12 \mathrm{w}, 460-465 \mathrm{~nm})$ as the heating source (the distance between the light source and Schlenk tube is $\sim 1.5 \mathrm{~cm})$ under irradiation of blue LED (12 w, $460-465$ $\mathrm{nm}$ ) for $48 \mathrm{~h}$. The reaction mixture was quenched by brine, extracted with ethyl acetate. The organic layer was dried over anhydrous $\mathrm{Na}_{2} \mathrm{SO}_{4}$, filtered and concentrated. The residue was purified with silica gel chromatography to give product 3 .

\subsection{General Procedure for Deaminative Alkylation of Difluoroenoxysilane 2a with Pyridinium Salts 4 (Procedure B)}<smiles></smiles>

4<smiles>[O-]C(O[Ga])=C(F)F</smiles>

2a

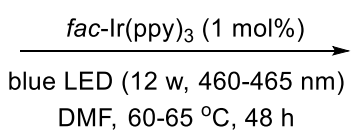

DMF, $60-65^{\circ} \mathrm{C}, 48 \mathrm{~h}$

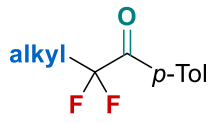

3

To a $25 \mathrm{~mL}$ of glass Schlenk tube were added pyridinium salt $4(0.5 \mathrm{mmol}, 1.0$ equiv. $)$ and $\mathrm{fac}$ - $\operatorname{Ir}(\mathrm{ppy})_{3}$ (3.27 mg, $0.005 \mathrm{mmol}, 1 \mathrm{~mol} \%$ ) under argon, followed by $\mathbf{2 a}$ (427 mg, $1.5 \mathrm{mmol}, 3$ equiv.) and DMF $(5 \mathrm{~mL})$. The reaction mixture was stirred at $60-65^{\circ} \mathrm{C}$ with blue LED $(12 \mathrm{w}, 460-465 \mathrm{~nm})$ as the heating source (the distance between the light source and Schlenk tube is $\sim 1.5 \mathrm{~cm}$ ) under irradiation of blue LED (12 w, $460-465 \mathrm{~nm}$ ) for $48 \mathrm{~h}$. The reaction mixture was quenched by brine, extracted with ether acetate. The organic layer was dried over anhydrous $\mathrm{Na}_{2} \mathrm{SO}_{4}$, filtered and concentrated. The residue was purified with silica gel chromatography to give product 3 . 


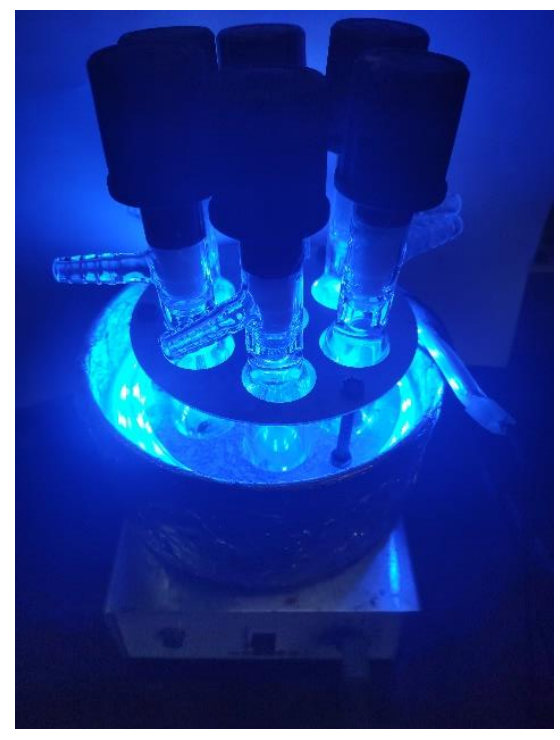

Figure S2. Synthesis of compound 3 with 1 mol\% photocatalyst.

\section{Characterization Data for Compounds 3.}

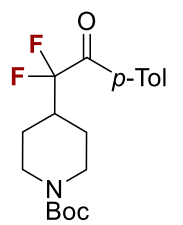

tert-Butyl 4-(1,1-difluoro-2-oxo-2-(p-tolyl)ethyl)piperidine-1-carboxylate (3a). The product (166 mg, 94\% yield, procedure A; $116 \mathrm{mg}, 89 \%$ yield, procedure B) as a yellow solid (m.p. 66-68 ${ }^{\circ} \mathrm{C}$ ) was purified by flash column chromatography (Hexane: Ethyl Acetate = 10: 1). ${ }^{1} \mathrm{H}$ NMR $\left(400 \mathrm{MHz}, \mathrm{CDCl}_{3}\right) \delta 7.97(\mathrm{~d}, J=8.0 \mathrm{~Hz}, 2 \mathrm{H}), 7.27(\mathrm{~d}, J=8.0$ Hz, 2 H), 4.18 (br, 2 H), 2.67 (br, 2 H), 2.50 - 2.32 (m, 4 H), 1.78 - 1.75 (m, 2 H), 1.53 - 1.44 (m, 11 $\mathrm{H}) .{ }^{19} \mathrm{~F}$ NMR $\left(376 \mathrm{MHz}, \mathrm{CDCl}_{3}\right) \delta-107.9(\mathrm{t}, J=13.3 \mathrm{~Hz}, 2 \mathrm{~F}) .{ }^{13} \mathrm{C} \mathrm{NMR}\left(100 \mathrm{MHz}, \mathrm{CDCl}_{3}\right) \delta 189.0$ $\left(\mathrm{t}, \mathrm{C}-\mathrm{F}, 2 J_{C-F}=30.3 \mathrm{~Hz}\right), 154.5,145.5,130.1\left(\mathrm{t}, \mathrm{C}-\mathrm{F}, 4 J_{C-F}=3.5 \mathrm{~Hz}\right), 129.9\left(\mathrm{t}, \mathrm{C}-\mathrm{F}, 3 J_{C-F}=1.9 \mathrm{~Hz}\right)$, $129.4,119.5\left(\mathrm{t}, \mathrm{C}-\mathrm{F}, 1 J_{C-F}=253.8 \mathrm{~Hz}\right), 79.6,43.0(\mathrm{br}), 40.3\left(\mathrm{t}, \mathrm{C}-\mathrm{F}, 2 J_{C-F}=22.1 \mathrm{~Hz}\right), 28.3,24.2,21.7$. MS (ESI): m/z (\%) 298 (100), 340, 376 [M+Na] $]^{+}$HRMS (ESI- quadrupole) m/z: [M+Na] ${ }^{+}$Calcd for $\mathrm{C}_{19} \mathrm{H}_{25} \mathrm{NO}_{3} \mathrm{~F}_{2} \mathrm{Na} 376.1700$; Found 376.1693.

Gram-scale synthesis of compound 3a with 0.01 mol\% catalyst. Redox-ester (1a) (1.50 g, 4 mmol, 1.0 equiv.) and a solution of $f a c-\operatorname{Ir}(\mathrm{ppy})_{3}$ in $\mathrm{DMF}\left(4 \times 10^{-4} \mathrm{M}, 1.0 \mathrm{~mL}, 0.26 \mathrm{mg}, 0.0004 \mathrm{mmol}, 0.01\right.$ mol\%) were added to a $100 \mathrm{~mL}$ of glass Schlenk tube. The mixture was evacuated and backfilled with argon for three times. Difluoroenoxysilane 2a (1.48 g, $5.2 \mathrm{mmol}, 1.3$ equiv.) and DMF (10 mL) were added. The reaction mixture stirred at $60{ }^{\circ} \mathrm{C}$ with blue LED $(12 \mathrm{w}, 460-465 \mathrm{~nm})$ as the heating source (the distance between the light source and Schlenk tube is $<1 \mathrm{~cm}$ ) under irradiation of blue LED (12 
w, $460-465 \mathrm{~nm})$. After stirring for $48 \mathrm{~h}$, the reaction mixture was diluted with ethyl acetate $(30 \mathrm{~mL})$. The reaction mixture was washed with brine. The organic layer was dried over anhydrous $\mathrm{Na}_{2} \mathrm{SO}_{4}$, filtered and concentrated. The residue was purified with silica gel chromatography (hexane: Ethyl Acetate $=10: 1)$ to give product 3a $(1.17 \mathrm{~g}, 84 \%$ yield $)$.

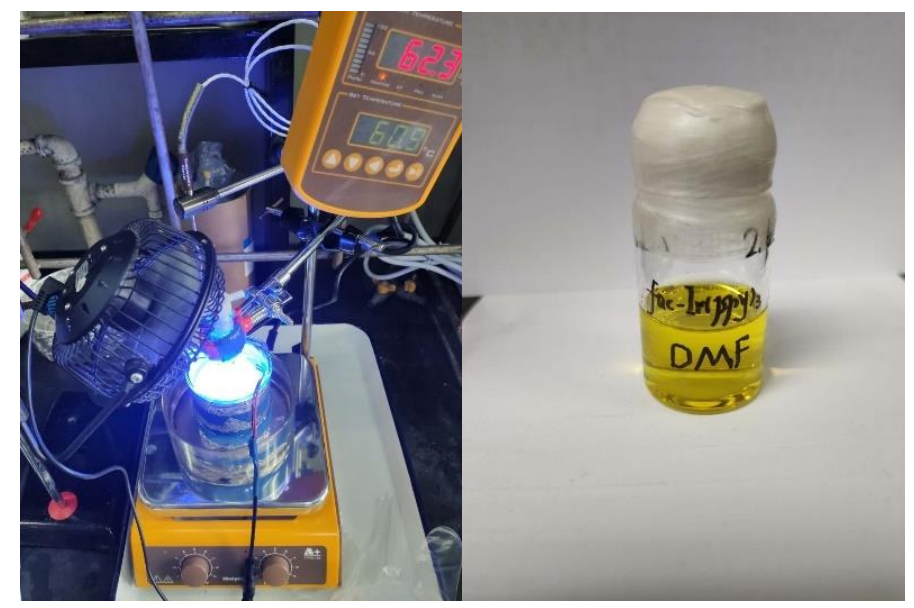

Figure S3. (left) Gram-scale synthesis of compound 3a with 0.01 mol\% catalyst. (right) A solution of $f a c-\operatorname{Ir}(\mathrm{ppy})_{3}$ in $\mathrm{DMF}\left(4 \times 10^{-4} \mathrm{M}\right)$

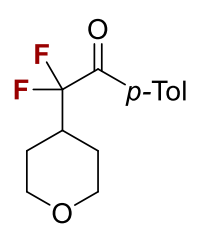

2,2-Difluoro-2-(tetrahydro-2H-pyran-4-yl)-1-(p-tolyl)ethan-1-one (3b). The product (103 mg, 81\% yield) as a colorless oil was prepared according to Procedure A and purified by flash column chromatography (Hexane: Ethyl Acetate = 20: 1). ${ }^{1} \mathrm{H}$ NMR (400 $\left.\mathrm{MHz}, \mathrm{CDCl}_{3}\right) \delta 7.99(\mathrm{~d}, J=8.0 \mathrm{~Hz}, 2 \mathrm{H}), 7.29(\mathrm{~d}, J=8.0 \mathrm{~Hz}, 2 \mathrm{H}), 4.02(\mathrm{~d}, J=11.2 \mathrm{~Hz}$, 2H), 3.55-3.26 (m, 2H), 2.62-2.46 (m, 1H), $2.42(\mathrm{~s}, 3 \mathrm{H}), 1.72-1.68(\mathrm{~m}, 4 \mathrm{H}) .{ }^{19} \mathrm{~F}$ NMR (376 MHz, $\left.\mathrm{CDCl}_{3}\right) \delta-108.6(\mathrm{~d}, J=15.0 \mathrm{~Hz}, 2 \mathrm{~F}) .{ }^{13} \mathrm{C} \mathrm{NMR}\left(100 \mathrm{MHz}, \mathrm{CDCl}_{3}\right) \delta 189.0\left(\mathrm{t}, \mathrm{C}-\mathrm{F}, 2 J_{C-F}=30.3 \mathrm{~Hz}\right)$, $145.5,130.1\left(\mathrm{t}, \mathrm{C}-\mathrm{F}, 4 J_{C-F}=3.6 \mathrm{~Hz}\right), 129.9\left(\mathrm{t}, \mathrm{C}-\mathrm{F}, 3 J_{C-F}=2.2 \mathrm{~Hz}\right), 129.4,119.3\left(\mathrm{t}, \mathrm{C}-\mathrm{F}, 1 J_{C-F}=253.6\right.$ $\mathrm{Hz}), 67.1,39.3$ (t, C-F, $\left.2 J_{C-F}=22.3 \mathrm{~Hz}\right), 24.9$ (t, C-F, 3J $\left.J_{C-F}=4.4 \mathrm{~Hz}\right), 21.8 . \mathrm{MS}(\mathrm{EI}): \mathrm{m} / \mathrm{z}(\%) 119.1$ (100), 170.1, $254.1[\mathrm{M}]^{+}$. HRMS (EI-TOF) m/z: [M] ${ }^{+}$Calcd for $\mathrm{C}_{14} \mathrm{H}_{16} \mathrm{O}_{2} \mathrm{~F}_{2}$ 254.1118; Found 254.1116. 
2-Cyclohexyl-2,2-difluoro-1-(p-tolyl)ethan-1-one (3c). The product (105 mg, 83\% yield) as a colorless oil was prepared according to Procedure A and purified by flash column chromatography (Hexane). ${ }^{1} \mathrm{H} \mathrm{NMR}\left(400 \mathrm{MHz}, \mathrm{CDCl}_{3}\right) \delta 7.98(\mathrm{~d}, J=8.0 \mathrm{~Hz}, 2 \mathrm{H}), 7.28$ $(\mathrm{d}, J=8.0 \mathrm{~Hz}, 2 \mathrm{H}), 2.43(\mathrm{~s}, 3 \mathrm{H}), 2.31-2.12(\mathrm{~m}, 1 \mathrm{H}), 1.87-1.73(\mathrm{~m}, 4 \mathrm{H}), 1.72-1.61(\mathrm{~m}, 1 \mathrm{H}), 1.34-$ $1.12(\mathrm{~m}, 5 \mathrm{H}) .{ }^{19} \mathrm{~F}$ NMR $\left(376 \mathrm{MHz}, \mathrm{CDCl}_{3}\right) \delta-108.6(\mathrm{~d}, J=15.0 \mathrm{~Hz}, 2 \mathrm{~F}) .{ }^{13} \mathrm{C} \mathrm{NMR}\left(100 \mathrm{MHz}, \mathrm{CDCl}_{3}\right)$ $\delta 189.9\left(\mathrm{t}, \mathrm{C}-\mathrm{F}, 2 J_{C-F}=29.8 \mathrm{~Hz}\right), 145.2,130.4\left(\mathrm{t}, \mathrm{C}-\mathrm{F}, 3 J_{C-F}=1.6 \mathrm{~Hz}\right), 130.1\left(\mathrm{t}, \mathrm{C}-\mathrm{F}, 4 J_{C-F}=3.6 \mathrm{~Hz}\right)$, 129.3, $120.4\left(\mathrm{t}, \mathrm{C}-\mathrm{F}, 1 J_{C-F}=252.7 \mathrm{~Hz}\right), 42.2\left(\mathrm{t}, \mathrm{C}-\mathrm{F}, 2 J_{C-F}=21.7 \mathrm{~Hz}\right), 25.9,25.4,24.8\left(\mathrm{t}, \mathrm{C}-\mathrm{F}, 3 J_{C-F}=\right.$ 4.2 Hz), 21.7. MS (EI): m/z (\%) 119.1 (100), 170.0, $252.1[\mathrm{M}]^{+}$. HRMS (EI-TOF) m/z: [M] Calcd for $\mathrm{C}_{15} \mathrm{H}_{18} \mathrm{OF}_{2} 252.1326$; Found 252.1318.

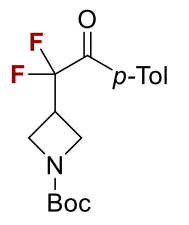

tert-Butyl 3-(1,1-difluoro-2-oxo-2-(p-tolyl)ethyl)azetidine-1-carboxylate (3d). The product (150 mg, 92\% yield) as a yellow solid (m.p. 104-106 $\left.{ }^{\circ} \mathrm{C}\right)$ was prepared according to Procedure A and purified by flash column chromatography (Hexane: Ethyl Acetate = 10: 1). ${ }^{1} \mathrm{H}$ NMR $\left(400 \mathrm{MHz}, \mathrm{CDCl}_{3}\right) \delta 8.01(\mathrm{~d}, J=8.0 \mathrm{~Hz}, 2 \mathrm{H}), 7.30(\mathrm{~d}, J=8.0 \mathrm{~Hz}, 2 \mathrm{H}), 4.13-4.07$ $(\mathrm{m}, 2 \mathrm{H}), 4.03-3.96(\mathrm{~m}, 2 \mathrm{H}), 3.39$ (br, 1H), 2.43 (s, 3H), 1.43 (s, 9H). ${ }^{19} \mathrm{~F}$ NMR $\left(376 \mathrm{MHz}, \mathrm{CDCl}_{3}\right) \delta$ $-108.64(\mathrm{dd}, J=301.4,12.8 \mathrm{~Hz}, 1 \mathrm{~F}),-108.93(\mathrm{dd}, J=301.4,15.4 \mathrm{~Hz}, 1 \mathrm{~F}) .{ }^{13} \mathrm{C} \mathrm{NMR}\left(100 \mathrm{MHz}, \mathrm{CDCl}_{3}\right)$ $\delta 187.7\left(\mathrm{t}, \mathrm{C}-\mathrm{F}, 2 J_{C-F}=31.6 \mathrm{~Hz}\right), 156.0,146.1,130.4\left(\mathrm{t}, \mathrm{C}-\mathrm{F}, 4 J_{C-F}=3.0 \mathrm{~Hz}\right), 129.5,128.9\left(\mathrm{t}, \mathrm{C}-\mathrm{F}, 3 J_{C-}\right.$ $\left.{ }_{F}=3.3 \mathrm{~Hz}\right), 118.4\left(\mathrm{t}, \mathrm{C}-\mathrm{F}, 1 J_{C-F}=253.8 \mathrm{~Hz}\right), 79.8,48.7(\mathrm{br}), 31.9\left(\mathrm{t}, \mathrm{C}-\mathrm{F}, 2 J_{C-F}=24.7 \mathrm{~Hz}\right), 28.3,21.9$. MS (ESI): m/z (\%) 270.1 (100), $348.1[\mathrm{M}+\mathrm{Na}]^{+}$. HRMS (ESI-quadrupole) m/z: $[\mathrm{M}+\mathrm{Na}]^{+}$Calcd for $\mathrm{C}_{17} \mathrm{H}_{21} \mathrm{NO}_{3} \mathrm{NaF}_{2}$ 348.1387; Found 348.1382.

Gram-Scale Synthesis of 3d. Redox-ester 1d (1.38 g, 4 mmol, 1.0 equiv.) and fac-Ir(ppy) 3 (26.3 mg, $0.04 \mathrm{mmol}, 1 \mathrm{~mol} \%$ ) were added to a $100 \mathrm{~mL}$ of glass Schlenk tube. The mixture was evacuated and backfilled with argon for three times. Compound 2a (3.41 g, 12 mmol, 3.0 equiv.) and DMF (40 mL) were added. The reaction mixture was stirred at $60{ }^{\circ} \mathrm{C}$ with blue LED $(12 \mathrm{w}, 460-465 \mathrm{~nm})$ as the heating source (the distance between the light source and Schlenk tube is $<1 \mathrm{~cm}$ ) under irradiation of blue LED (12 w, $460-465 \mathrm{~nm})$. After stirring for $48 \mathrm{~h}$, the reaction mixture was diluted with ethyl acetate $(30 \mathrm{~mL})$. The reaction mixture was washed with brine. The organic layer was dried over anhydrous $\mathrm{Na}_{2} \mathrm{SO}_{4}$, filtered and concentrated. The residue was purified with silica gel chromatography 
to give product $\mathbf{3 d}(1.27 \mathrm{~g}, 98 \%$ yield).

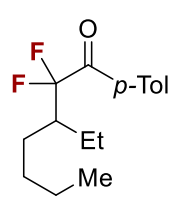

3-Ethyl-2,2-difluoro-1-(p-tolyl)heptan-1-one (3e). The product (107 mg, 80\% yield) as a colorless oil was prepared according to Procedure A and purified by flash column chromatography (Hexane). ${ }^{1} \mathrm{H}$ NMR $\left(400 \mathrm{MHz}, \mathrm{CDCl}_{3}\right) \delta 7.98(\mathrm{~d}, J=8.0 \mathrm{~Hz}, 2 \mathrm{H}), 7.28$ $(\mathrm{d}, J=8.0 \mathrm{~Hz}, 2 \mathrm{H}), 2.42(\mathrm{~s}, 3 \mathrm{H}), 2.29-2.15(\mathrm{~m}, 1 \mathrm{H}), 1.71-1.52(\mathrm{~m}, 2 \mathrm{H}), 1.51-1.34(\mathrm{~m}, 3 \mathrm{H}), 1.34$ $-1.23(\mathrm{~m}, 3 \mathrm{H}), 0.96(\mathrm{t}, J=7.6 \mathrm{~Hz}, 3 \mathrm{H}), 0.88(\mathrm{t}, J=7.2 \mathrm{~Hz}, 3 \mathrm{H}) .{ }^{19} \mathrm{~F} \mathrm{NMR}\left(376 \mathrm{MHz}, \mathrm{CDCl}_{3}\right) \delta-104.5$ $(\mathrm{dd}, J=273.2,16.7 \mathrm{~Hz}, 1 \mathrm{~F}),-105.3(\mathrm{dd}, J=273.2,16.7 \mathrm{~Hz}, 1 \mathrm{~F}) .{ }^{13} \mathrm{C} \mathrm{NMR}\left(100 \mathrm{MHz}, \mathrm{CDCl}_{3}\right) \delta 189.9$ $\left(\mathrm{t}, \mathrm{C}-\mathrm{F}, 2 J_{C-F}=30.0 \mathrm{~Hz}\right), 145.1,130.3\left(\mathrm{t}, \mathrm{C}-\mathrm{F}, 3 J_{C-F}=2.9 \mathrm{~Hz}\right), 130.1\left(\mathrm{t}, \mathrm{C}-\mathrm{F}, 4 J_{C-F}=3.5 \mathrm{~Hz}\right), 129.3$, $121.5\left(\mathrm{t}, \mathrm{C}-\mathrm{F}, 1 J_{C-F}=254.2 \mathrm{~Hz}\right), 43.7\left(\mathrm{t}, \mathrm{C}-\mathrm{F}, 2 J_{C-F}=20.4 \mathrm{~Hz}\right), 29.5,26.6\left(\mathrm{t}, \mathrm{C}-\mathrm{F}, 3 J_{C-F}=3.8 \mathrm{~Hz}\right), 22.9$, 21.7, 20.4 (t, C-F, 3J $\left.J_{C-F}=4.2 \mathrm{~Hz}\right), 13.8,11.7 . \mathrm{MS}(\mathrm{EI}): \mathrm{m} / \mathrm{z}(\%) 119.1(100), 176.1,268.1[\mathrm{M}]^{+}$. HRMS (EI-TOF) m/z: [M] $]^{+}$Calcd for $\mathrm{C}_{16} \mathrm{H}_{22} \mathrm{OF}_{2} 268.1639$; Found 268.1636.

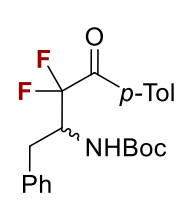

tert-Butyl (3,3-difluoro-4-oxo-1-phenyl-4-(p-tolyl)butan-2-yl)carbamate (3f). The product (158 mg, 81\% yield) as a yellow solid (m.p. 94-96 ${ }^{\circ} \mathrm{C}$ ) was prepared according to Procedure A and purified by flash column chromatography (Hexane: Ether Acetate $=20$ : 1). (Note: The compound is a rotameric mixture, and the resonances for minor rotamer are enclosed in parenthesis). ${ }^{1} \mathrm{H} \mathrm{NMR}\left(400 \mathrm{MHz}, \mathrm{CDCl}_{3}\right) \delta 8.01(\mathrm{~d}, J=8.0 \mathrm{~Hz}, 2 \mathrm{H}), 7.30(\mathrm{~d}, J=8.0 \mathrm{~Hz}, 2 \mathrm{H}), 7.28-$ 7.25 (m, 2H), $7.24-7.18(\mathrm{~m}, 3 \mathrm{H}), 4.85-4.48(\mathrm{~m}, 2 \mathrm{H}), 3.19-3.08(\mathrm{~m}, 1 \mathrm{H}), 2.83-2.73(\mathrm{~m}, 1 \mathrm{H}), 2.44$ $(\mathrm{s}, 3 \mathrm{H}), 1.27(\mathrm{~s}, 9 \mathrm{H}) .{ }^{19} \mathrm{~F}$ NMR $\left(376 \mathrm{MHz}, \mathrm{CDCl}_{3}\right) \delta$ major rotamer: $-107.5(\mathrm{~d}, J=11.3 \mathrm{~Hz}, 1.58 \mathrm{~F})$, minor rotamer: - 106.4, $(\mathrm{d}, J=281.8 \mathrm{~Hz}, 0.21 \mathrm{~F}),-110.4,(\mathrm{~d}, J=281.8 \mathrm{~Hz}, 0.21 \mathrm{~F}) .{ }^{13} \mathrm{C}$ NMR $(100$ $\left.\mathrm{MHz}, \mathrm{CDCl}_{3}\right) \delta 188.5\left(\mathrm{t}, \mathrm{C}-\mathrm{F}, 2 J_{C-F}=29.2 \mathrm{~Hz}\right), 154.9(154.3), 145.8$, (136.6) 136.3, $130.2\left(\mathrm{t}, \mathrm{C}-\mathrm{F}, 4 J_{C-}\right.$ $F=3.0 \mathrm{~Hz}),(129.7) 129.5$, (129.4) 129.2, 128.5, $126.7,117.4\left(\mathrm{t}, \mathrm{C}-\mathrm{F}, 1 J_{C-F}=258.8 \mathrm{~Hz}\right),(80.4) 79.9$, 54.5 (t, C-F, $\left.2 J_{C-F}=24.5 \mathrm{~Hz}\right),(35.4)$ 35.1, 28.1 (27.6), 21.9. MS (ESI): m/z (\%) 290.1 (100), 334.1, $412.2[\mathrm{M}+\mathrm{Na}]^{+}$. HRMS (ESI-quadrupole) $\mathrm{m} / \mathrm{z}$ : $[\mathrm{M}+\mathrm{Na}]^{+}$Calcd for $\mathrm{C}_{22} \mathrm{H}_{25} \mathrm{~F}_{2} \mathrm{NO}_{3} \mathrm{Na}$ 412.1700; Found 412.1694 .

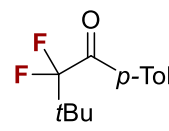

2,2-Difluoro-3,3-dimethyl-1-(p-tolyl)butan-1-one (3g). The product ( $80 \mathrm{mg}, 71 \%$ yield) as a colorless oil was prepared according to Procedure A and purified by flash column 
chromatography (Hexane). ${ }^{1} \mathrm{H}$ NMR $\left(400 \mathrm{MHz}, \mathrm{CDCl}_{3}\right) \delta 7.99(\mathrm{~d}, J=8.0 \mathrm{~Hz}, 2 \mathrm{H}), 7.27(\mathrm{~d}, J=8.0 \mathrm{~Hz}$, 2H), $2.42(\mathrm{~s}, 3 \mathrm{H}), 1.14(\mathrm{~s}, 9 \mathrm{H}) .{ }^{19} \mathrm{~F}$ NMR $\left(376 \mathrm{MHz}, \mathrm{CDCl}_{3}\right) \delta-108.8$ (s, 2F). ${ }^{13} \mathrm{C} \mathrm{NMR}(100 \mathrm{MHz}$, $\left.\mathrm{CDCl}_{3}\right) \delta 190.3\left(\mathrm{t}, \mathrm{C}-\mathrm{F}, 2 J_{C-F}=30.7 \mathrm{~Hz}\right), 144.9,131.9\left(\mathrm{t}, \mathrm{C}-\mathrm{F}, 3 J_{C-F}=2.1 \mathrm{~Hz}\right), 130.4\left(\mathrm{t}, \mathrm{C}-\mathrm{F}, 4 J_{C-F}=\right.$ $4.4 \mathrm{~Hz}), 129.3,122.0\left(\mathrm{t}, \mathrm{C}-\mathrm{F}, 1 J_{C-F}=256.2 \mathrm{~Hz}\right), 37.7$ (t, C-F, 2J $\left.J_{C-F}=21.6 \mathrm{~Hz}\right), 24.1$ (t, C-F, 3J $J_{C-F}=$ 4.2 Hz), 21.7. MS (EI): m/z (\%) 119.1 (100), 170.1, $226.1[\mathrm{M}]^{+}$. HRMS (EI-TOF) m/z: [M] Calcd for $\mathrm{C}_{13} \mathrm{H}_{16} \mathrm{OF}_{2} 226.1169$; Found 226.1161.

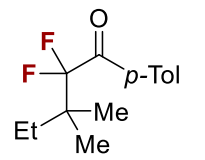

2,2-Difluoro-3,3-dimethyl-1-(p-tolyl)pentan-1-one (3h). The product (80 mg, 67\% yield) as a colorless oil was prepared according to Procedure A and purified by flash column chromatography (Hexane). ${ }^{1} \mathrm{H}$ NMR $\left(400 \mathrm{MHz}, \mathrm{CDCl}_{3}\right) \delta 7.98(\mathrm{~d}, J=8.0 \mathrm{~Hz}, 2 \mathrm{H}), 7.26(\mathrm{~d}, J$ $=8.0 \mathrm{~Hz}, 2 \mathrm{H}), 2.42(\mathrm{~s}, 3 \mathrm{H}), 1.56(\mathrm{q}, J=7.6 \mathrm{~Hz}, 2 \mathrm{H}), 1.08(\mathrm{~s}, 6 \mathrm{H}), 0.89(\mathrm{t}, J=7.6 \mathrm{~Hz}, 3 \mathrm{H}) .{ }^{19} \mathrm{~F} \mathrm{NMR}$ $\left(376 \mathrm{MHz}, \mathrm{CDCl}_{3}\right) \delta-107.6(\mathrm{~s}, 2 \mathrm{~F}) .{ }^{13} \mathrm{C} \mathrm{NMR}\left(100 \mathrm{MHz}, \mathrm{CDCl}_{3}\right) \delta 190.6\left(\mathrm{t}, \mathrm{C}-\mathrm{F}, 2 J_{C-F}=30.7 \mathrm{~Hz}\right)$, 144.8, $132.1\left(\mathrm{t}, \mathrm{C}-\mathrm{F}, 3 J_{C-F}=1.9 \mathrm{~Hz}\right), 130.4\left(\mathrm{t}, \mathrm{C}-\mathrm{F}, 4 J_{C-F}=4.5 \mathrm{~Hz}\right), 129.2,122.5\left(\mathrm{t}, \mathrm{C}-\mathrm{F}, 1 J_{C-F}=256.9\right.$ $\mathrm{Hz}), 40.7$ (t, C-F, $2 J_{C-F}=20.4 \mathrm{~Hz}$ ), $28.4\left(\mathrm{t}, \mathrm{C}-\mathrm{F}, 3 J_{C-F}=3.5 \mathrm{~Hz}\right), 21.7,20.4$ (t, C-F, $\left.3 J_{C-F}=4.4 \mathrm{~Hz}\right), 8.0$. MS (EI): m/z (\%) 119.1 (100), 170.1, 240.1 [M] $]^{+}$HRMS (EI-TOF) m/z: [M] Calcd for $\mathrm{C}_{14} \mathrm{H}_{18} \mathrm{OF}_{2}$ 240.1326; Found 240.1319.

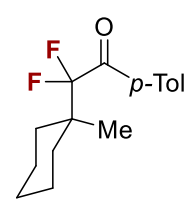

2,2-Difluoro-2-(1-methylcyclohexyl)-1-(p-tolyl)ethan-1-one (3i). The product (122 mg, 92\% yield) as a colorless oil was prepared according to Procedure A and purified by flash column chromatography (Hexane). ${ }^{1} \mathrm{H}$ NMR (400 MHz, $\left.\mathrm{CDCl}_{3}\right) \delta 7.97$ (d, $J=8.4 \mathrm{~Hz}$, 2H), $7.24(\mathrm{~d}, J=8.4 \mathrm{~Hz}, 2 \mathrm{H}), 2.40(\mathrm{~s}, 3 \mathrm{H}), 1.67-1.49(\mathrm{~m}, 7 \mathrm{H}), 1.47-1.32(\mathrm{~m}, 2 \mathrm{H}), 1.12(\mathrm{~s}, 3 \mathrm{H}), 1.09$ - $1.01(\mathrm{~m}, 1 \mathrm{H}) .{ }^{19} \mathrm{~F}$ NMR $\left(376 \mathrm{MHz}, \mathrm{CDCl}_{3}\right) \delta-110.7(\mathrm{~s}, 2 \mathrm{~F}) .{ }^{13} \mathrm{C} \mathrm{NMR}\left(100 \mathrm{MHz}, \mathrm{CDCl}_{3}\right) \delta 190.7(\mathrm{t}$, $\left.\mathrm{C}-\mathrm{F}, 2 J_{C-F}=30.4 \mathrm{~Hz}\right), 144.7,132.3\left(\mathrm{t}, \mathrm{C}-\mathrm{F}, 3 J_{C-F}=1.9 \mathrm{~Hz}\right), 130.4\left(\mathrm{t}, \mathrm{C}-\mathrm{F}, 4 J_{C-F}=4.6 \mathrm{~Hz}\right), 129.2,122.3$ $\left(\mathrm{t}, \mathrm{C}-\mathrm{F}, 1 J_{C-F}=256.6 \mathrm{~Hz}\right), 40.8\left(\mathrm{t}, \mathrm{C}-\mathrm{F}, 2 J_{C-F}=20.3 \mathrm{~Hz}\right), 30.0\left(\mathrm{t}, \mathrm{C}-\mathrm{F}, 3 J_{C-F}=4.0 \mathrm{~Hz}\right), 25.6,21.6,20.9$, 16.7 (t, C-F, $\left.3 J_{C-F}=4.0 \mathrm{~Hz}\right)$. MS (EI): m/z (\%) $83(100), 119,266[\mathrm{M}]^{+}$. HRMS (EI-TOF) m/z: [M] $]^{+}$ Calcd for $\mathrm{C}_{16} \mathrm{H}_{20} \mathrm{~F}_{2} \mathrm{O} 266.1477$; Found 266.1472.

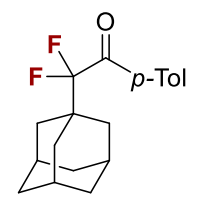

2-(Adamantan-1-yl)-2,2-difluoro-1-(p-tolyl)ethan-1-one (3j). The product (109 mg, $72 \%$ yield) as a colorless oil was prepared according to Procedure A and purified by 
flash column chromatography (Hexane: Ether Acetate $=80: 1) .{ }^{1} \mathrm{H}$ NMR $\left(400 \mathrm{MHz}, \mathrm{CDCl}_{3}\right) \delta 7.99(\mathrm{~d}$, $J=8.0 \mathrm{~Hz}, 2 \mathrm{H}), 7.26(\mathrm{~d}, J=8.0 \mathrm{~Hz}, 2 \mathrm{H}), 2.42(\mathrm{~s}, 3 \mathrm{H}), 2.01(\mathrm{~s}, 3 \mathrm{H}), 1.79(\mathrm{~d}, J=2.4 \mathrm{~Hz}, 6 \mathrm{H}), 1.67$ (q, $J=12.1 \mathrm{~Hz}, 6 \mathrm{H}) .{ }^{19} \mathrm{~F}$ NMR $\left(376 \mathrm{MHz}, \mathrm{CDCl}_{3}\right) \delta-113.4(\mathrm{~s}, 2 \mathrm{~F}) .{ }^{13} \mathrm{C} \mathrm{NMR}\left(100 \mathrm{MHz}, \mathrm{CDCl}_{3}\right) \delta 190.7$ $\left(\mathrm{t}, \mathrm{C}-\mathrm{F}, 2 J_{C-F}=29.6 \mathrm{~Hz}\right), 144.9,132.3\left(\mathrm{t}, \mathrm{C}-\mathrm{F}, 3 J_{C-F}=1.6 \mathrm{~Hz}\right), 130.4\left(\mathrm{t}, \mathrm{C}-\mathrm{F}, 4 J_{C-F}=4.6 \mathrm{~Hz}\right), 129.2$, $121.6\left(\mathrm{t}, \mathrm{C}-\mathrm{F}, 1 J_{C-F}=254.8 \mathrm{~Hz}\right), 39.4\left(\mathrm{t}, \mathrm{C}-\mathrm{F}, 2 J_{C-F}=21.6 \mathrm{~Hz}\right), 36.5,35.2\left(\mathrm{t}, \mathrm{C}-\mathrm{F}, 3 J_{C-F}=3.6 \mathrm{~Hz}\right), 27.6$, 21.7. MS (EI): m/z (\%) 119.1 (100), $304[\mathrm{M}]^{+}$. HRMS (EI-TOF) m/z: [M] Calcd for $\mathrm{C}_{19} \mathrm{H}_{22} \mathrm{OF}_{2}$ 304.1639; Found 304.1632.

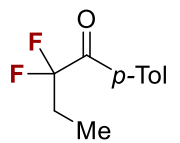

2,2-Difluoro-1-(p-tolyl)butan-1-one (3k). The product ( $87 \mathrm{mg}, 88 \%$ yield) as a colorless oil was prepared according to Procedure A and purified by flash column chromatography (Hexane). ${ }^{1} \mathrm{H}$ NMR (400 MHz, $\left.\mathrm{CDCl}_{3}\right) \delta 8.01$ (d, $\left.J=8.0 \mathrm{~Hz}, 2 \mathrm{H}\right), 7.29$ (d, $\left.J=8.0 \mathrm{~Hz}, 2 \mathrm{H}\right), 2.43$ (s, $3 \mathrm{H}), 2.28-2.14(\mathrm{~m}, 2 \mathrm{H}), 1.09(\mathrm{t}, J=7.4 \mathrm{~Hz}, 3 \mathrm{H}) .{ }^{19} \mathrm{~F}$ NMR $\left(376 \mathrm{MHz}, \mathrm{CDCl}_{3}\right) \delta-102.2(\mathrm{t}, J=17.5$ $\mathrm{Hz}, 2 \mathrm{~F}) .{ }^{13} \mathrm{C}$ NMR $\left(100 \mathrm{MHz}, \mathrm{CDCl}_{3}\right) \delta 189.2\left(\mathrm{t}, \mathrm{C}-\mathrm{F}, 2 J_{C-F}=31.3 \mathrm{~Hz}\right), 145.3,130.3\left(\mathrm{t}, \mathrm{C}-\mathrm{F}, 4 J_{C-F}=\right.$ $3.4 \mathrm{~Hz}), 129.6\left(\mathrm{t}, \mathrm{C}-\mathrm{F}, 3 J_{C-F}=2.4 \mathrm{~Hz}\right), 129.4,120.1\left(\mathrm{t}, \mathrm{C}-\mathrm{F}, 1 J_{C-F}=251.0 \mathrm{~Hz}\right), 27.5\left(\mathrm{t}, \mathrm{C}-\mathrm{F}, 2 J_{C-F}=\right.$ 23.6 Hz), 21.8, 5.8 (t, C-F, 3J $\left.J_{C-F}=5.6 \mathrm{~Hz}\right)$. MS (EI): m/z (\%) 119.1 (100), $198.1[\mathrm{M}]^{+}$. HRMS (EITOF) m/z: [M] $]^{+}$Calcd for $\mathrm{C}_{11} \mathrm{H}_{12} \mathrm{OF}_{2}$ 198.0856; Found 198.0849.

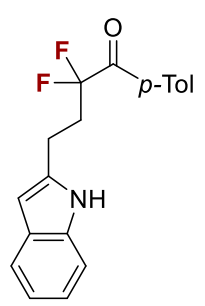

2,2-Difluoro-4-(1H-indol-3-yl)-1-(p-tolyl)butan-1-one (31). The product (94 mg, 60\% yield) as a brown oil was prepared according to Procedure A and purified by flash column chromatography (Hexane: Ethyl Acetate = 10: 1). ${ }^{1} \mathrm{H}$ NMR $\left(400 \mathrm{MHz}, \mathrm{CDCl}_{3}\right)$ $\delta 8.04(\mathrm{~d}, J=8.0 \mathrm{~Hz}, 2 \mathrm{H}), 7.97(\mathrm{br}, 1 \mathrm{H}), 7.60(\mathrm{~d}, J=8.0 \mathrm{~Hz}, 1 \mathrm{H}), 7.35(\mathrm{~d}, J=8.0 \mathrm{~Hz}$, 1H), $7.29(\mathrm{~d}, J=8.0 \mathrm{~Hz}, 2 \mathrm{H}), 7.22-7.17(\mathrm{~m}, 1 \mathrm{H}), 7.14-7.09(\mathrm{~m}, 1 \mathrm{H}), 7.03-7.00$ (m, 1H), $3.06-$ $3.00(\mathrm{~m}, 2 \mathrm{H}), 2.67-2.54(\mathrm{~m}, 2 \mathrm{H}), 2.44(\mathrm{~s}, 3 \mathrm{H}) .{ }^{19} \mathrm{~F} \mathrm{NMR}\left(376 \mathrm{MHz}, \mathrm{CDCl}_{3}\right) \delta-100.5(\mathrm{t}, J=17.3 \mathrm{~Hz}$, 2F). ${ }^{13} \mathrm{C}$ NMR $\left(125 \mathrm{MHz}, \mathrm{CDCl}_{3}\right) \delta 188.9\left(\mathrm{t}, \mathrm{C}-\mathrm{F}, 2 J_{C-F}=30.6 \mathrm{~Hz}\right), 145.5,136.3,130.3$ (t, C-F, $4 J_{C-F}$ $=3.3 \mathrm{~Hz}), 129.6,129.4,127.1,122.1,121.4,119.4,118.7,117.7\left(\mathrm{t}, \mathrm{C}-\mathrm{F}, 1 J_{C-F}=261.6 \mathrm{~Hz}\right), 114.6,111.2$, $35.0\left(\mathrm{t}, \mathrm{C}-\mathrm{F}, 2 J_{C-F}=22.5 \mathrm{~Hz}\right), 21.9,17.5$ (t, C-F, $\left.3 J_{C-F}=5.5 \mathrm{~Hz}\right) . \mathrm{MS}(\mathrm{EI}): \mathrm{m} / \mathrm{z}(\%) 119$ (100), 143, $313[\mathrm{M}]^{+}$. HRMS (EI-TOF) m/z: [M] ${ }^{+}$Calcd for $\mathrm{C}_{19} \mathrm{H}_{17} \mathrm{~F}_{2} \mathrm{NO} 313.1278$; Found 313.1280. 


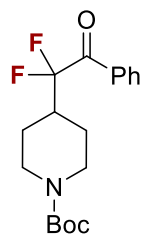

tert-Butyl 4-(1,1-difluoro-2-oxo-2-phenylethyl)piperidine-1-carbox-ylate $(3 \mathrm{~m})$. The product (163 mg, 96\% yield) as a colorless oil was prepared according to Procedure A and purified by flash column chromatography (Hexane: Ethyl Acetate = 15: 1). ${ }^{1} \mathrm{H}$ NMR (400 $\left.\mathrm{MHz}, \mathrm{CDCl}_{3}\right) \delta 8.08-8.06(\mathrm{~m}, 2 \mathrm{H}), 7.66-7.59(\mathrm{~m}, 1 \mathrm{H}), 7.54-7.43(\mathrm{~m}, 2 \mathrm{H}), 4.19(\mathrm{br}, 2 \mathrm{H}), 2.68$ (br, 2H), $2.51-2.36(\mathrm{~m}, 1 \mathrm{H}), 1.79-1.75(\mathrm{~m}, 2 \mathrm{H}), 1.53-1.46(\mathrm{~m}, 2 \mathrm{H}), 1.44(\mathrm{~s}, 9 \mathrm{H}) .{ }^{19} \mathrm{~F}$ NMR $(376 \mathrm{MHz}$, $\left.\mathrm{CDCl}_{3}\right) \delta-107.9(\mathrm{t}, J=13.9 \mathrm{~Hz}, 2 \mathrm{~F}) .{ }^{13} \mathrm{C} \mathrm{NMR}\left(100 \mathrm{MHz}, \mathrm{CDCl}_{3}\right) \delta 189.5\left(\mathrm{t}, \mathrm{C}-\mathrm{F}, 2 J_{C-F}=30.7 \mathrm{~Hz}\right)$, $154.6,134.4,132.5\left(\mathrm{t}, \mathrm{C}-\mathrm{F}, 3 J_{C-F}=2.0 \mathrm{~Hz}\right), 130.1\left(\mathrm{t}, \mathrm{C}-\mathrm{F}, 4 J_{C-F}=3.5 \mathrm{~Hz}\right), 128.7,119.5\left(\mathrm{t}, \mathrm{C}-\mathrm{F}, 1 J_{C-F}=\right.$ 253.7 Hz), 79.7, 43.1 (br), 40.3 (t, C-F, $\left.2 J_{C-F}=22.0 \mathrm{~Hz}\right), 28.4,24.3 . \mathrm{MS}(\mathrm{EI}): \mathrm{m} / \mathrm{z}(\%) 57.1$ (100), 105, 219.1, 266.1, $339.1[\mathrm{M}]^{+}$. HRMS (EI-TOF) m/z: [M] ${ }^{+}$Calcd for $\mathrm{C}_{18} \mathrm{H}_{23} \mathrm{NO}_{3} \mathrm{~F}_{2}$ 339.1646; Found 339.1643.

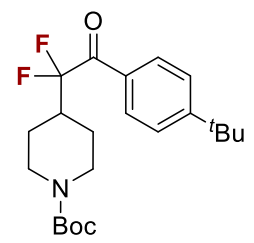

tert-Butyl 4-(2-(4-(tert-butyl)phenyl)-1,1-difluoro-2-oxoethyl)-piperidine-1carboxylate (3n). The product (152 mg, 77\% yield) as a yellow solid (m.p. 83-85 $\left.{ }^{\circ} \mathrm{C}\right)$ was prepared according to Procedure A and purified by flash column chromatography $($ Hexane: Ethyl Acetate $=15: 1) .{ }^{1} \mathrm{H}$ NMR $\left(400 \mathrm{MHz}, \mathrm{CDCl}_{3}\right) \delta 8.02(\mathrm{~d}, J=8.4 \mathrm{~Hz}, 2 \mathrm{H}), 7.50(\mathrm{~d}, J=$ $8.4 \mathrm{~Hz}, 2 \mathrm{H}), 4.19$ (br, 2H), 2.68 (br, 2H), 2.50 - 2.36 (m, 1H), 1.79-1.76 (m, 2H), 1.53-1.47 (m, 2H), $1.44(\mathrm{~s}, 9 \mathrm{H}), 1.33(\mathrm{~s}, 9 \mathrm{H}) .{ }^{19} \mathrm{~F}$ NMR $\left(376 \mathrm{MHz}, \mathrm{CDCl}_{3}\right) \delta-107.9(\mathrm{t}, J=13.0 \mathrm{~Hz}, 2 \mathrm{~F}) .{ }^{13} \mathrm{C}$ NMR $(100$ $\left.\mathrm{MHz}, \mathrm{CDCl}_{3}\right) \delta 189.1\left(\mathrm{t}, \mathrm{C}-\mathrm{F}, 2 J_{C-F}=30.3 \mathrm{~Hz}\right), 158.4,154.6,130.1\left(\mathrm{t}, \mathrm{C}-\mathrm{F}, 4 J_{C-F}=3.4 \mathrm{~Hz}\right), 129.9(\mathrm{t}$, $\left.\mathrm{C}-\mathrm{F}, 3 J_{C-F}=2.2 \mathrm{~Hz}\right), 125.8,119.6\left(\mathrm{t}, \mathrm{C}-\mathrm{F}, 1 J_{C-F}=253.8 \mathrm{~Hz}\right), 79.7,43.1(\mathrm{br}), 40.4\left(\mathrm{t}, \mathrm{C}-\mathrm{F}, 2 J_{C-F}=22.0\right.$ $\mathrm{Hz}), 35.3,31.0,28.4,24.3 . \mathrm{MS}(\mathrm{ESI}): \mathrm{m} / \mathrm{z}(\%) 340$ (100), 418 [M+Na] ${ }^{+}$. HRMS (ESI-quadrupole) m/z: $[\mathrm{M}+\mathrm{Na}]^{+}$Calcd for $\mathrm{C}_{22} \mathrm{H}_{31} \mathrm{NO}_{3} \mathrm{NaF}_{2} 418.2170$; Found 418.2161.

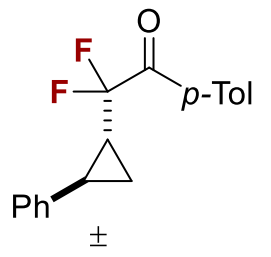

2,2-Difluoro-2-(2-phenylcyclopropyl)-1-(p-tolyl)ethan-1-one (3o). The product

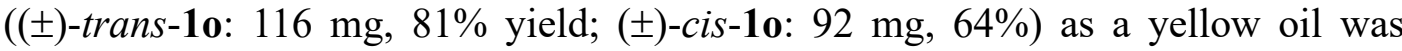
prepared according to Procedure A and purified by flash column chromatography (Hexane: Ethyl Acetate = 80: 1). ${ }^{1} \mathrm{H}$ NMR $\left(400 \mathrm{MHz}, \mathrm{CDCl}_{3}\right) \delta 8.05(\mathrm{~d}, J=8.0 \mathrm{~Hz}$, 2H), $7.36-7.25(\mathrm{~m}, 4 \mathrm{H}), 7.24-7.12(\mathrm{~m}, 3 \mathrm{H}), 2.44(\mathrm{~s}, 3 \mathrm{H}), 2.42-2.38(\mathrm{~m}, 1 \mathrm{H}), 1.99-1.85(\mathrm{~m}, 1 \mathrm{H})$, $1.45-1.36(\mathrm{~m}, 1 \mathrm{H}), 1.23-1.13(\mathrm{~m}, 1 \mathrm{H}) .{ }^{19} \mathrm{~F}$ NMR $\left(376 \mathrm{MHz}, \mathrm{CDCl}_{3}\right) \delta-103.6(\mathrm{dd}, J=276.6,12.1$ $\mathrm{Hz}, 1 \mathrm{~F}),-105.1(\mathrm{dd}, J=276.6,13.2 \mathrm{~Hz}, 1 \mathrm{~F}) .{ }^{13} \mathrm{C} \mathrm{NMR}\left(125 \mathrm{MHz}, \mathrm{CDCl}_{3}\right) \delta 188.6\left(\mathrm{t}, \mathrm{C}-\mathrm{F}, 2 J_{C-F}=31.9\right.$ 
$\mathrm{Hz}), 145.5,140.0,130.4\left(\mathrm{t}, \mathrm{C}-\mathrm{F}, 4 J_{C-F}=3.2 \mathrm{~Hz}\right), 129.4,128.5,126.6,126.4,117.9$ (t, C-F, $1 J_{C-F}=$ $250.5 \mathrm{~Hz}), 24.0$ (t, C-F, $\left.2 J_{C-F}=26.9 \mathrm{~Hz}\right), 21.8,19.5,10.9$. MS (EI): m/z (\%) 119.1 (100), $286[\mathrm{M}]^{+}$. HRMS (EI-TOF) m/z: [M] Calcd for $\mathrm{C}_{18} \mathrm{H}_{16} \mathrm{OF}_{2} 286.1169$; Found 286.1160.

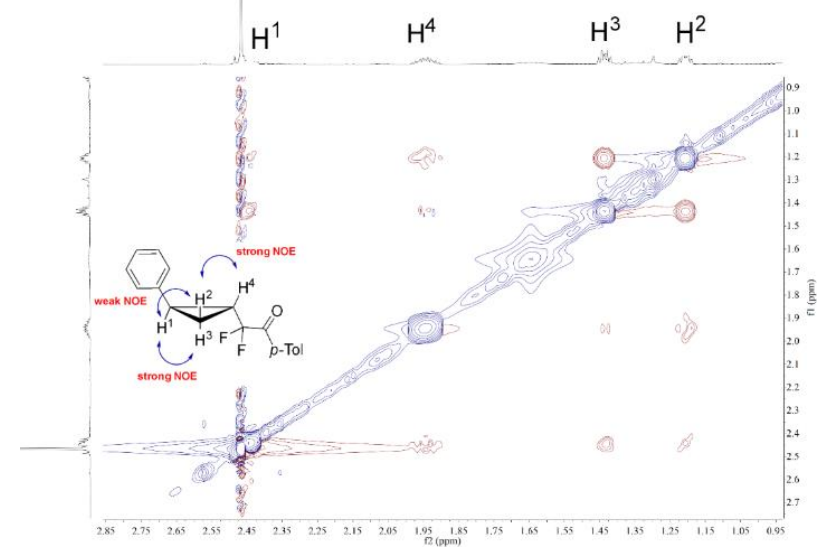

Figure S4. NOE Spectrum of Compound 3o

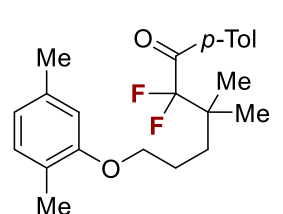

6-(2,5-Dimethylphenoxy)-2,2-difluoro-3,3-dim-ethyl-1-(p-tolyl)hexan-1-one

(3p). The product (178 mg, 95\% yield) as a yellow oil was prepared according to Procedure A and purified by flash column chromatography (Hexane: Ethyl Acetate = 80: 1). ${ }^{1} \mathrm{H} \mathrm{NMR}\left(400 \mathrm{MHz}, \mathrm{CDCl}_{3}\right) \delta 8.03(\mathrm{~d}, J=8.0 \mathrm{~Hz}, 2 \mathrm{H}), 7.29(\mathrm{~d}, J=8.0 \mathrm{~Hz}, 2 \mathrm{H}), 7.03(\mathrm{~d}, J=$ $7.2 \mathrm{~Hz}, 1 \mathrm{H}), 6.70(\mathrm{~d}, J=7.6 \mathrm{~Hz}, 1 \mathrm{H}), 6.65(\mathrm{~s}, 1 \mathrm{H}), 3.95(\mathrm{t}, J=6.2 \mathrm{~Hz}, 2 \mathrm{H}), 2.45(\mathrm{~s}, 3 \mathrm{H}), 2.35(\mathrm{~s}, 3 \mathrm{H})$, $2.20(\mathrm{~s}, 3 \mathrm{H}), 1.93-1.85(\mathrm{~m}, 2 \mathrm{H}), 1.81-1.73(\mathrm{~m}, 2 \mathrm{H}), 1.21(\mathrm{~s}, 6 \mathrm{H}) .{ }^{19} \mathrm{~F} \mathrm{NMR}\left(376 \mathrm{MHz}, \mathrm{CDCl}_{3}\right) \delta-$ $107.1(\mathrm{~s}, 2 \mathrm{~F}) .{ }^{13} \mathrm{C} \mathrm{NMR}\left(100 \mathrm{MHz}, \mathrm{CDCl}_{3}\right) \delta 190.3\left(\mathrm{t}, \mathrm{C}-\mathrm{F}, 2 J_{C-F}=30.8 \mathrm{~Hz}\right), 156.9,144.8,136.4,131.9$ $\left(\mathrm{t}, \mathrm{C}-\mathrm{F}, 3 J_{C-F}=2.0 \mathrm{~Hz}\right), 130.3\left(\mathrm{t}, \mathrm{C}-\mathrm{F}, 4 J_{C-F}=4.5 \mathrm{~Hz}\right), 130.2,129.2,123.5,122.2\left(\mathrm{t}, \mathrm{C}-\mathrm{F}, 1 J_{C-F}=257.3\right.$ $\mathrm{Hz}), 120.7,111.9,68.0,40.4\left(\mathrm{t}, \mathrm{C}-\mathrm{F}, 2 J_{C-F}=20.6 \mathrm{~Hz}\right), 32.6\left(\mathrm{t}, \mathrm{C}-\mathrm{F}, 3 J_{C-F}=3.3 \mathrm{~Hz}\right), 24.0,21.621 .3$, $21.1\left(\mathrm{t}, \mathrm{C}-\mathrm{F}, 3 J_{C-F}=4.4 \mathrm{~Hz}\right), 15.7 . \mathrm{MS}(\mathrm{EI}): \mathrm{m} / \mathrm{z}(\%) 119$ (100), 253, 374 [M] $]^{+}$HRMS (EI-TOF) m/z: $[\mathrm{M}]^{+}$Calcd for $\mathrm{C}_{23} \mathrm{H}_{28} \mathrm{O}_{2} \mathrm{~F}_{2}$ 374.2057; Found 374.2056.

Gram-Scale Synthesis of 3p. Redox-ester (1p) (1.38 g, 3.5 mmol, 1.0 equiv.) and fac-Ir(ppy) ${ }_{3}(23.02$ $\mathrm{mg}, 0.035 \mathrm{mmol}, 1 \mathrm{~mol} \%$ ) were added to a $100 \mathrm{~mL}$ of glass Schlenk tube. The mixture was evacuated and backfilled with argon for three times. Compound 2a (2.98 g, $10.5 \mathrm{mmol}, 3.0$ equiv.) and DMF (35 $\mathrm{mL})$ were added. The reaction mixture was stirred at $60{ }^{\circ} \mathrm{C}$ with blue LED $(12 \mathrm{w}, 460-465 \mathrm{~nm})$ as the heating source (the distance between the light source and Schlenk tube is $<1 \mathrm{~cm}$ ) under irradiation of 
blue LED (12 w, 460 - $465 \mathrm{~nm})$. After stirring for $48 \mathrm{~h}$, the reaction mixture was diluted with ethyl acetate $(30 \mathrm{~mL})$. The reaction mixture was washed with brine. The organic layer was dried over anhydrous $\mathrm{Na}_{2} \mathrm{SO}_{4}$, filtered and concentrated. The residue was purified with silica gel chromatography to give product $3 \mathbf{p}(1.24 \mathrm{~g}, 95 \%$ yield).

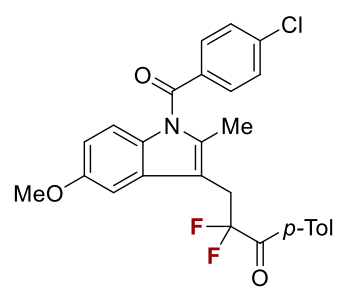

\section{3-(1-(4-Chlorobenzoyl)-5-methoxy-2-methyl-1H-indol-3-yl)-2,2-difluoro-} 1-(p-tolyl)propan-1-one (3q). The product (190 mg, $79 \%$ yield) as a yellow solid (m.p. 82-84 ${ }^{\circ} \mathrm{C}$ ) was prepared according to Procedure A and purified by flash column chromatography (Hexane: Ethyl Acetate =10: 1). ${ }^{1} \mathrm{H}$ NMR (400 $\left.\mathrm{MHz} \mathrm{CDCl}_{3}\right) \delta 7.95(\mathrm{~d}, J=8.0 \mathrm{~Hz}, 2 \mathrm{H}), 7.57(\mathrm{~d}, J=8.8 \mathrm{~Hz}, 2 \mathrm{H}), 7.44(\mathrm{~d}, J=8.8 \mathrm{~Hz}, 2 \mathrm{H}), 7.26-7.24$ $(\mathrm{m}, 2 \mathrm{H}), 6.96(\mathrm{~s}, 1 \mathrm{H}), 6.91(\mathrm{~d}, J=9.2 \mathrm{~Hz}, 1 \mathrm{H}), 6.68(\mathrm{dd}, J=9.2,2.4 \mathrm{~Hz}, 1 \mathrm{H}), 3.81(\mathrm{~s}, 3 \mathrm{H}), 3.58(\mathrm{t}, J$ $=17.6 \mathrm{~Hz}, 2 \mathrm{H}), 2.41(\mathrm{~s}, 3 \mathrm{H}), 2.29(\mathrm{~s}, 3 \mathrm{H}) .{ }^{19} \mathrm{~F} \mathrm{NMR}\left(376 \mathrm{MHz}, \mathrm{CDCl}_{3}\right) \delta-97.3(\mathrm{t}, J=17.6 \mathrm{~Hz}, 2 \mathrm{~F})$. ${ }^{13} \mathrm{C} \mathrm{NMR}\left(100 \mathrm{MHz}, \mathrm{CDCl}_{3}\right) \delta 189.3\left(\mathrm{t}, \mathrm{C}-\mathrm{F}, 2 J_{C-F}=30.2 \mathrm{~Hz}\right), 168.2,156.0,145.6,139.3,137.4,133.8$, 131.2, 131.14, 131.11, 130.8, $130.2\left(\mathrm{t}, \mathrm{C}-\mathrm{F}, 4 J_{C-F}=3.2 \mathrm{~Hz}\right), 129.6\left(\mathrm{t}, \mathrm{C}-\mathrm{F}, 3 J_{C-F}=2.3 \mathrm{~Hz}\right), 129.4,129.1$, $119.0\left(\mathrm{t}, \mathrm{C}-\mathrm{F}, 1 J_{C-F}=253.5 \mathrm{~Hz}\right), 114.8,111.9,109.8\left(\mathrm{t}, \mathrm{C}-\mathrm{F}, 3 J_{C-F}=3.6 \mathrm{~Hz}\right), 101.5,55.6,29.6(\mathrm{t}, \mathrm{C}-\mathrm{F}$, $\left.2 J_{C-F}=24.8 \mathrm{~Hz}\right), 21.8,13.5 . \mathrm{MS}(\mathrm{ESI}): \mathrm{m} / \mathrm{z}(\%) 482.0\left([\mathrm{M}+\mathrm{H}]^{+}, 100\right)$. HRMS (ESI-quadrupole) $\mathrm{m} / \mathrm{z}$ : $[\mathrm{M}+\mathrm{H}]^{+}$Calcd for $\mathrm{C}_{27} \mathrm{H}_{23} \mathrm{ClNO}_{3} \mathrm{~F}_{2}$ 482.1335; Found 482.1327 .

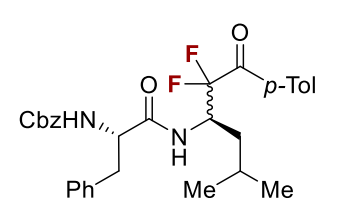

Benzyl ((2S)-1-((2,2-difluoro-5-methyl-1-oxo-1-(p-tolyl)hexan-3-yl)amino)1-oxo-3-phenylpropan-2-yl)carbamate (3s). The product (193 mg, 72\% yield, diastereomer) as a white solid (m.p. $144.3-146.1^{\circ} \mathrm{C}$ ) was prepared according to Procedure A and purified by flash column chromatography (Hexane: Ethyl Acetate = 5: 1). (Note: The product is a stereoisomer $(\mathrm{dr}=1: 1)$, and the resonances for minor isomer are enclosed in parenthesis). ${ }^{1} \mathrm{H}$ NMR $\left(400 \mathrm{MHz}, \mathrm{CDCl}_{3}\right) \delta 7.97(\mathrm{~d}, J=7.6 \mathrm{~Hz}, 2 \mathrm{H}), 7.41-7.27(\mathrm{~m}, 7 \mathrm{H}), 7.24-7.04$ (m, 5H), $6.22-6.01(\mathrm{~s}, 1 \mathrm{H}), 5.35(\mathrm{~d}, J=7.3 \mathrm{~Hz}, 1 \mathrm{H}), 5.16-5.01(\mathrm{~s}, 2 \mathrm{H}), 4.81(\mathrm{~d}, J=7.4 \mathrm{~Hz}, 1 \mathrm{H})$, $4.43(\mathrm{~d}, J=16.6 \mathrm{~Hz}, 1 \mathrm{H}), 3.15-2.89(\mathrm{~m}, 2 \mathrm{H}), 2.43$ (d, $J=7.7 \mathrm{~Hz}, 3 \mathrm{H}), 1.59-1.40$ (m, 1H), $1.40-$ $1.20(\mathrm{~m}, 2 \mathrm{H}), 0.90-0.83(\mathrm{~m}, 3 \mathrm{H}), 0.82-0.71(\mathrm{~d}, J=3.0 \mathrm{~Hz}, 3 \mathrm{H}) .{ }^{19} \mathrm{~F}$ NMR $\left(376 \mathrm{MHz}, \mathrm{CDCl}_{3}\right): \delta-$ $105.0(-106.3)(\mathrm{d}, J=288.0 \mathrm{~Hz}, 1 \mathrm{~F}),-108.6(-107.0)(\mathrm{d}, J=288.0 \mathrm{~Hz}, 1 \mathrm{~F}) .{ }^{13} \mathrm{C} \mathrm{NMR}\left(100 \mathrm{MHz}, \mathrm{CDCl}_{3}\right)$ $\delta 188.36\left(\mathrm{t}, \mathrm{C}-\mathrm{F}, 2 J_{C-F}=28.99 \mathrm{~Hz}\right)(188.19)\left(\mathrm{t}, \mathrm{C}-\mathrm{F}, 2 J_{C-F}=29.83 \mathrm{~Hz}\right),(171.02) 171.04,155.90$ 
(155.87), 145.92 (145.89), 136.24 (136.05), 130.25 (t, C-F, $\left.4 J_{C-F}=3.2 \mathrm{~Hz}\right)(130.16)\left(\mathrm{t}, \mathrm{C}-\mathrm{F}, 4 J_{C-F}=\right.$ $3.1 \mathrm{~Hz}), 129.52$ (129.50), 129.28 (129.22), 128.76, 128.66, 128.54, 128.21, 128.04, 127.98, 127.07 (127.02), 117.1 (t, C-F, $\left.1 J_{C-F}=257.5 \mathrm{~Hz}\right), 67.12,56.31,50.24$, (38.29) 38.08, 37.44 (37.18), 24.42, 24.16, 23.37 (23.33), 21.85 (21.83), 21.11 (21.07). MS (ESI): m/z (\%) $537\left([\mathrm{M}+\mathrm{H}]^{+}, 100\right)$. HRMS (ESI-quadrupole) m/z: [M+H] $]^{+}$Calcd for $\mathrm{C}_{31} \mathrm{H}_{35} \mathrm{~F}_{2} \mathrm{~N}_{2} \mathrm{O}_{4}$ 537.2565; Found 537.2560.

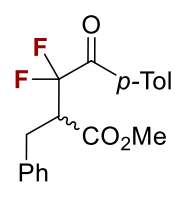

Methyl 2-benzyl-3,3-difluoro-4-oxo-4-(p-tolyl)butanoate (3t). The product (105 mg, $63 \%$ yield) as a colorless oil was prepared according to Procedure B and purified by flash column chromatography (Hexane: Ethyl Acetate = 10: 1). ${ }^{1} \mathrm{H}$ NMR (400 MHz, $\left.\mathrm{CDCl}_{3}\right) \delta 8.03(\mathrm{~d}, J=8.0 \mathrm{~Hz}, 2 \mathrm{H}), 7.35-7.29(\mathrm{~m}, 4 \mathrm{H}), 7.27-7.21(\mathrm{~m}, 3 \mathrm{H}), 3.80-3.65(\mathrm{~m}, 1 \mathrm{H}), 3.58$ (s, 3H), $3.20(\mathrm{~d}, J=7.2 \mathrm{~Hz}, 2 \mathrm{H}), 2.45(\mathrm{~s}, 3 \mathrm{H}) .{ }^{19} \mathrm{~F} \mathrm{NMR}\left(376 \mathrm{MHz}, \mathrm{CDCl}_{3}\right) \delta-101.9$ (dd, $J=294.0$, $13.9 \mathrm{~Hz}, 1 \mathrm{~F}),-103.3(\mathrm{dd}, J=294.0,15.0 \mathrm{~Hz}, 1 \mathrm{~F}) .{ }^{13} \mathrm{C} \mathrm{NMR}\left(100 \mathrm{MHz}, \mathrm{CDCl}_{3}\right) \delta 188.0\left(\mathrm{t}, \mathrm{C}-\mathrm{F}, 2 J_{C-F}\right.$ $=30.1 \mathrm{~Hz}), 169.5,145.8,137.5,130.4,129.5,129.2,128.9,128.6,126.9,117.7\left(\mathrm{t}, \mathrm{C}-\mathrm{F}, 1 J_{C-F}=257.8\right.$ Hz), 52.1, $51.6\left(\mathrm{t}, \mathrm{C}-\mathrm{F}, 2 J_{C-F}=21.6 \mathrm{~Hz}\right), 31.8,21.8 . \mathrm{MS}(\mathrm{EI}): \mathrm{m} / \mathrm{z}(\%) 119(100), 253,332[\mathrm{M}]^{+} . \mathrm{HRMS}$ (EI-TOF) m/z: [M] $]^{+}$Calcd for $\mathrm{C}_{19} \mathrm{H}_{18} \mathrm{O}_{3} \mathrm{~F}_{2}$ 332.1219; Found 332.1214.

\section{Transformation of Compounds 3.}

\subsection{Synthesis of Difluoromethylated Alkanes 5}

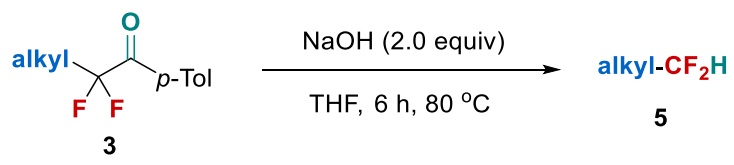

Procedure: Compound 3 (1.0 equiv), $\mathrm{NaOH}$ (2.0 equiv) and THF (2 mL) were added to a flask. After the reaction was stirred at $80^{\circ} \mathrm{C}$ for $6 \mathrm{~h}$, the resulting mixture was filtered. The filtrate was concentrated. The residue was purified by flash column chromatography on silica gel to give product $\mathbf{5}$.

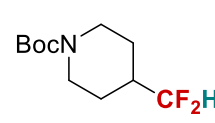

tert-Butyl 4-(difluoromethyl)piperidine-1-carboxylate (5a). The reaction was purified by flash column chromatography (hexane: Ethyl Acetate $=10: 1) .{ }^{1} \mathrm{H} \mathrm{NMR}\left(400 \mathrm{MHz}, \mathrm{CDCl}_{3}\right)$ $\delta 5.57(\mathrm{td}, J=56.4, J=5.2 \mathrm{~Hz}, 1 \mathrm{H}), 4.16(\mathrm{br}, 2 \mathrm{H}), 2.69(\mathrm{br}, 2 \mathrm{H}), 1.98-1.83(\mathrm{~m}, 1 \mathrm{H}), 1.75-1.68(\mathrm{~m}$, 
2H), $1.45(\mathrm{~s}, 9 \mathrm{H}), 1.39-1.26(\mathrm{~m}, 2 \mathrm{H}) .{ }^{19} \mathrm{~F}$ NMR (376 MHz, $\left.\mathrm{CDCl}_{3}\right) \delta-123.4(\mathrm{dd}, J=29.3,13.2 \mathrm{~Hz}$, $1 \mathrm{~F}),-123.51(\mathrm{dd}, J=29.3,12.7 \mathrm{~Hz}, 1 \mathrm{~F}) .{ }^{13} \mathrm{C} \mathrm{NMR}\left(100 \mathrm{MHz}, \mathrm{CDCl}_{3}\right) \delta 154.6,118.2\left(\mathrm{t}, \mathrm{C}-\mathrm{F}, 1 J_{C-F}=\right.$ $240.2 \mathrm{~Hz}$ ), 79.7, 42.7 (br), 40.2 (t, C-F, $\left.2 J_{C-F}=20.1 \mathrm{~Hz}\right), 28.4,24.6$ (t, C-F, 3 $\left.J_{C-F}=4.7 \mathrm{~Hz}\right) . \mathrm{MS}(\mathrm{EI})$ : m/z (\%) 57 (100), 134, 235 [M] $]^{+}$HRMS (EI- TOF) m/z: [M] Calcd for $\mathrm{C}_{11} \mathrm{H}_{19} \mathrm{~F}_{2} \mathrm{NO}_{2}$ 235.1384; Found 235.1385.

BocN $\beth_{\mathrm{CF}_{2} \mathrm{H}}^{\text {tert-Butyl }}$ 3-(difluoromethyl)azetidine-1-carboxylate $\mathbf{( 5 b ) . ~ T h e ~ r e a c t i o n ~ w a s ~}$
conducted on $0.2 \mathrm{mmol}$ scale. The product $\mathbf{5 b}(22 \mathrm{mg}, 52 \%$ yield $)$ as a colorless oil was purified by flash column chromatography (hexane: Ethyl Acetate $=15: 1) .{ }^{1} \mathrm{H} \mathrm{NMR}\left(400 \mathrm{MHz}, \mathrm{CDCl}_{3}\right)$ $\delta 5.94(\mathrm{td}, J=56.2,4.4 \mathrm{~Hz}, 1 \mathrm{H}), 4.05-3.97(\mathrm{~m}, 2 \mathrm{H}), 3.90(\mathrm{dd}, J=8.8,5.2 \mathrm{~Hz}, 2 \mathrm{H}), 2.98-2.85(\mathrm{~m}$, 1H), $1.43(\mathrm{~s}, 9 \mathrm{H}) .{ }^{19} \mathrm{~F}$ NMR $\left(376 \mathrm{MHz}, \mathrm{CDCl}_{3}\right) \delta-124.2(\mathrm{t}, J=59.7 \mathrm{~Hz}, 2 \mathrm{~F}) .{ }^{13} \mathrm{C} \mathrm{NMR}(125 \mathrm{MHz}$, $\left.\mathrm{CDCl}_{3}\right) \delta 156.1,116.1\left(\mathrm{t}, \mathrm{C}-\mathrm{F}, 1 J_{C-F}=239.0 \mathrm{~Hz}\right), 79.9,48.3,31.5\left(\mathrm{t}, \mathrm{C}-\mathrm{F}, 2 J_{C-F}=24.4 \mathrm{~Hz}\right), 28.3 . \mathrm{MS}$ (ESI): $\mathrm{m} / \mathrm{z}(\%)$ 152, $230\left([\mathrm{M}+\mathrm{Na}]^{+}, 100\right)$. HRMS (ESI-quadrupole) $\mathrm{m} / \mathrm{z}:[\mathrm{M}+\mathrm{Na}]^{+}$Calcd for $\mathrm{C}_{9} \mathrm{H}_{15} \mathrm{~F}_{2} \mathrm{NO}_{2} \mathrm{Na} 230.0969$; Found 230.0960.

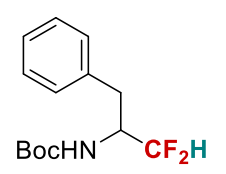

tert-Butyl (1,1-difluoro-3-phenylpropan-2-yl) carbamate (5c). The reaction was conducted on $0.15 \mathrm{mmol}$ scale. The product $5 \mathbf{c}$ (34 $\mathrm{mg}, 83 \%$ yield) as a white solid (m.p. 90-92 ${ }^{\circ} \mathrm{C}$ ) was purified by flash column chromatography (hexane: Ethyl Acetate $=20$ : 1). ${ }^{1} \mathrm{H}$ NMR (400 MHz, $\left.\mathrm{CDCl}_{3}\right) \delta 7.33-7.27$ (m, 2H), $7.26-7.18(\mathrm{~m}, 3 \mathrm{H}), 5.79(\mathrm{t}, J=55.6 \mathrm{~Hz}, 1 \mathrm{H})$, 4.64 (br, 1H), 4.20 (br, 1H), 2.99 (dd, $J=14.4,6.0 \mathrm{~Hz}, 1 \mathrm{H}), 2.85-2.75$ (m, 1H), 1.37 (s, 9H). ${ }^{19} \mathrm{~F}$ NMR (376 MHz, $\left.\mathrm{CDCl}_{3}\right) \delta-128.3(\mathrm{ddd}, J=281.6,55.6,12.4 \mathrm{~Hz}, 1 \mathrm{~F}),-130.5$ (ddd, $J=281.6,56.0$, $15.8 \mathrm{~Hz}, 1 \mathrm{~F}) .{ }^{13} \mathrm{C} \mathrm{NMR}\left(100 \mathrm{MHz}, \mathrm{CDCl}_{3}\right) \delta 155.2,136.1,129.2,128.7,126.9,114.8\left(\mathrm{t}, \mathrm{C}-\mathrm{F}, 1 J_{C-F}=\right.$ $242.8 \mathrm{~Hz}), 80.1,53.2\left(\mathrm{t}, \mathrm{C}-\mathrm{F}, 2 J_{C-F}=21.6 \mathrm{~Hz}\right), 34.1,28.2 . \mathrm{MS}(\mathrm{ESI}): \mathrm{m} / \mathrm{z}(\%) 294\left([\mathrm{M}+\mathrm{Na}]^{+}, 100\right)$. HRMS (ESI-quadrupole) m/z: [M+Na] ${ }^{+}$Calcd for $\mathrm{C}_{14} \mathrm{H}_{19} \mathrm{~F}_{2} \mathrm{NO}_{2} \mathrm{Na} 294.1276$; Found 294.1272.

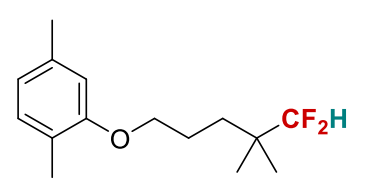

2-(4,4-Difluoro-3,3-dimethylbutoxy)-1,4-dimethylbenzene $\quad(5 \mathrm{~d}) . \quad$ The reaction was conducted on $0.2 \mathrm{mmol}$ scale. The product $\mathbf{5 d}$ (26 mg, 50\% yield) as a colorless oil was purified by flash column chromatography (hexane: Ethyl Acetate = 80: 1). ${ }^{1} \mathrm{H} \mathrm{NMR}\left(400 \mathrm{MHz}, \mathrm{CDCl}_{3}\right) \delta 7.02(\mathrm{~d}, J=7.3 \mathrm{~Hz}, 1 \mathrm{H}), 6.68(\mathrm{~d}, J=7.4 \mathrm{~Hz}, 1 \mathrm{H})$, 
$6.63(\mathrm{~s}, 1 \mathrm{H}), 5.57(\mathrm{t}, J=57.0 \mathrm{~Hz}, 1 \mathrm{H}), 3.94(\mathrm{t}, J=6.2 \mathrm{~Hz}, 2 \mathrm{H}), 2.32(\mathrm{~s}, 3 \mathrm{H}), 2.19(\mathrm{~s}, 3 \mathrm{H}), 1.86-1.76$ $(\mathrm{m}, 2 \mathrm{H}), 1.58-1.51(\mathrm{~m}, 2 \mathrm{H}), 1.02(\mathrm{~s}, 6 \mathrm{H}) .{ }^{19} \mathrm{~F} \mathrm{NMR}\left(376 \mathrm{MHz}, \mathrm{CDCl}_{3}\right) \delta-133.4(\mathrm{~d}, J=57.0 \mathrm{~Hz}, 2 \mathrm{~F})$. ${ }^{13} \mathrm{C} \mathrm{NMR}\left(125 \mathrm{MHz}, \mathrm{CDCl}_{3}\right) \delta 156.9,136.5,130.4,123.6,120.74\left(\mathrm{t}, \mathrm{C}-\mathrm{F}, 1 J_{C-F}=249.9 \mathrm{~Hz}\right), 120.76$, 111.9, 68.1, 37.7 (t, C-F, $\left.2 J_{C-F}=17.9 \mathrm{~Hz}\right), 32.6,23.7,21.4,20.3,15.8 . \mathrm{MS}(\mathrm{EI}): \mathrm{m} / \mathrm{z}(\%) 122(100)$, $256[\mathrm{M}]^{+}$. HRMS (EI-TOF) m/z: [M] $]^{+}$Calcd for $\mathrm{C}_{15} \mathrm{H}_{22} \mathrm{~F}_{2} \mathrm{O} 256.1639$; Found 256.1640.

\subsection{Synthesis of $\alpha, \alpha$-difluoroalkyl carboxylic ester 6}

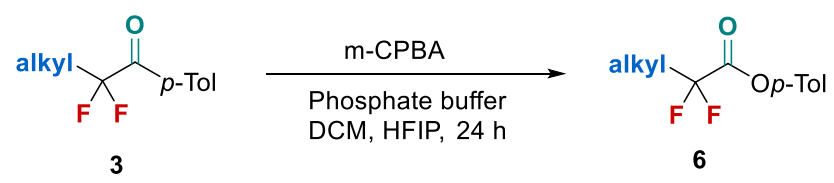

General Procedure: Compound 3 (1.0 equiv) was dissolved in $\mathrm{CH}_{2} \mathrm{Cl}_{2} / \mathrm{HFIP}$ (5:2, v/v, $\left.2.8 \mathrm{~mL}\right) . \mathrm{m}$ CPBA (10 equiv) and phosphate buffer $(150.0 \mu \mathrm{L})$ were then added. After the reaction was stirred at room temperature for $24 \mathrm{~h}$, the reaction mixture was quenched with saturated aqueous $\mathrm{Na}_{2} \mathrm{~S}_{2} \mathrm{O}_{3}$. The resulting mixture was extracted with EtOAc and washed with saturated aqueous $\mathrm{NaHCO}_{3}$ for three times. The combined organic layers were dried over anhydrous $\mathrm{Na}_{2} \mathrm{SO}_{4}$ and concentrated. The residue was purified with flash column chromatography to give product $\mathbf{6}$.

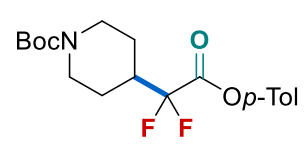

tert-Butyl 4-(1,1-difluoro-2-oxo-2-(p-tolyloxy) ethyl) piperidine-1-carboxylate

(6a). The reaction was conducted on $0.2 \mathrm{mmol}$ scale. The product $\mathbf{6 a}$ ( $46 \mathrm{mg}, 63 \%$ yield) as a colorless oil was purified by flash column chromatography (hexane: Ethyl Acetate = 20: 1). ${ }^{1} \mathrm{H}$ NMR $\left(400 \mathrm{MHz}, \mathrm{CDCl}_{3}\right) \delta 7.21(\mathrm{~d}, J=8.0 \mathrm{~Hz}, 2 \mathrm{H}), 7.02(\mathrm{~d}, J=8.4 \mathrm{~Hz}$, 2H), 4.24 (br, 2H), 2.72 (br, 2H), $2.47-2.40(\mathrm{~m}, 1 \mathrm{H}), 2.36$ (s, 3H), 1.83 (d, J=12.8 Hz, 2H), 1.60 $1.51(\mathrm{~m}, 2 \mathrm{H}), 1.46(\mathrm{~s}, 9 \mathrm{H}) .{ }^{19} \mathrm{~F}$ NMR $\left(376 \mathrm{MHz}, \mathrm{CDCl}_{3}\right) \delta-113.0(\mathrm{dd}, J=34.2,13.5 \mathrm{~Hz}, 2 \mathrm{~F}) .{ }^{13} \mathrm{C} \mathrm{NMR}$ $\left(125 \mathrm{MHz} \mathrm{CDCl}_{3}\right) \delta 162.5\left(\mathrm{t}, \mathrm{C}-\mathrm{F}, 2 J_{C-F}=33.2 \mathrm{~Hz}\right), 154.6,147.5,136.7,130.2,120.5,116.6(\mathrm{t}, \mathrm{C}-\mathrm{F}$, $\left.1 J_{C-F}=252.0 \mathrm{~Hz}\right), 79.9,42.9(\mathrm{br}), 41.0\left(\mathrm{t}, \mathrm{C}-\mathrm{F}, 2 J_{C-F}=22.5 \mathrm{~Hz}\right), 28.4,24.2,20.9 . \mathrm{MS}(\mathrm{ESI}): \mathrm{m} / \mathrm{z}(\%)$ 314 (100), $392[\mathrm{M}+\mathrm{Na}]^{+}$. HRMS (ESI-quadrupole) $\mathrm{m} / \mathrm{z}$ : $[\mathrm{M}+\mathrm{Na}]^{+} \mathrm{Calcd}$ for $\mathrm{C}_{19} \mathrm{H}_{25} \mathrm{~F}_{2} \mathrm{NO}_{4} \mathrm{Na} 392.1644$; Found 392.1638. 
p-Tolyl 3-((tert-butoxycarbonyl)amino)-2,2-difluoro-4-phenylbutanoate (6b). The reaction was

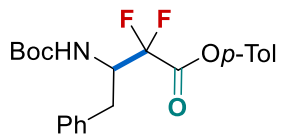

conducted on $0.2 \mathrm{mmol}$. The product $\mathbf{6 b}$ (69 $\mathrm{mg}, 85 \%$ yield) as a colorless oil was purified by flash column chromatography (hexane: Ethyl Acetate $=20: 1$ ). ${ }^{1} \mathrm{H}$ NMR $\left(400 \mathrm{MHz}, \mathrm{CDCl}_{3}\right) \delta 7.35-7.28(\mathrm{~m}, 2 \mathrm{H}), 7.26-7.21(\mathrm{~m}, 3 \mathrm{H}), 7.17(\mathrm{~d}, J=8.0 \mathrm{~Hz}, 2 \mathrm{H}), 7.03(\mathrm{~d}, J=8.4$ $\mathrm{Hz}, 2 \mathrm{H}), 4.87-4.71(\mathrm{~m}, 1 \mathrm{H}), 4.64(\mathrm{~d}, J=10.5 \mathrm{~Hz}, 1 \mathrm{H}), 3.30-3.23(\mathrm{~m}, 1 \mathrm{H}), 2.81-2.72(\mathrm{~m}, 1 \mathrm{H}), 2.35$ (s, 3H), $1.28(\mathrm{~s}, 9 \mathrm{H}) .{ }^{19} \mathrm{~F}$ NMR $\left(376 \mathrm{MHz}, \mathrm{CDCl}_{3}\right) \delta-110.8(\mathrm{dd}, J=256.8,20.3 \mathrm{~Hz}, 1 \mathrm{~F}),-120.1$ (dd, $J$ $=256.8,20.3 \mathrm{~Hz}, 1 \mathrm{~F}) .{ }^{13} \mathrm{C} \mathrm{NMR}\left(100 \mathrm{MHz}, \mathrm{CDCl}_{3}\right) \delta 161.8\left(\mathrm{t}, \mathrm{C}-\mathrm{F}, 2 J_{C-F}=30.8 \mathrm{~Hz}\right), 154.7,147.6$, $136.5,135.6,130.0,129.3,128.6,126.9,120.7,114.7\left(\mathrm{t}, \mathrm{C}-\mathrm{F}, 1 J_{C-F}=255.7 \mathrm{~Hz}\right), 80.3,53.8(\mathrm{t}, \mathrm{C}-\mathrm{F}$, $\left.2 J_{C-F}=25.3 \mathrm{~Hz}\right), 33.9,28.0,20.8 . \mathrm{MS}(\mathrm{ESI}): \mathrm{m} / \mathrm{z}(\%) 428\left([\mathrm{M}+\mathrm{Na}]^{+}, 100\right)$. HRMS (ESI-quadrupole) $\mathrm{m} / \mathrm{z}:[\mathrm{M}+\mathrm{Na}]^{+}$Calcd for $\mathrm{C}_{22} \mathrm{H}_{25} \mathrm{~F}_{2} \mathrm{NO}_{4} \mathrm{Na} 428.1649$; Found 428.1641 .

\subsection{Reduction of ketone 3a}

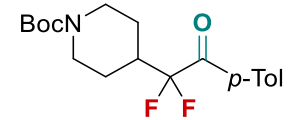

$3 \mathbf{a}$

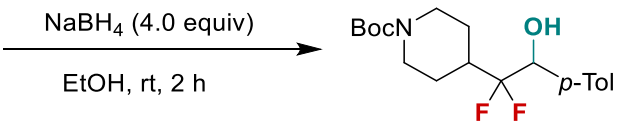

7, $73 \%$

To a solution of compound 3a (74 mg, $0.20 \mathrm{mmol}, 1.0 \mathrm{eq})$ in EtOH (2 mL) was added $\mathrm{NaBH}_{4}(30.4$ $\mathrm{mg}, 0.8 \mathrm{mmol}, 4.0$ equiv) at room temperature. After the reaction was stirred for $2 \mathrm{~h}$, aqueous solution $\mathrm{HCl}(1 \mathrm{M})$ was added. The resulting mixture was extracted with ethyl acetate. The combined organic layers were dried over anhydrous $\mathrm{Na}_{2} \mathrm{SO}_{4}$ and concentrated. The crude mixture was purified by silica gel column chromatography (hexane: Ethyl Acetate $=5: 1)$ to give product $7(53 \mathrm{mg}, 73 \%$ yield $)$ a colorless oil. ${ }^{1} \mathrm{H}$ NMR $\left(400 \mathrm{MHz}, \mathrm{CDCl}_{3}\right) \delta 7.31(\mathrm{~d}, J=8.0 \mathrm{~Hz}, 2 \mathrm{H}), 7.18(\mathrm{~d}, J=8.0 \mathrm{~Hz}, 2 \mathrm{H}), 4.81(\mathrm{t}$, $J=11.4 \mathrm{~Hz}, 1 \mathrm{H}), 4.1$ (br, 2H), 2.98 (br, 1H), 2.56 (br, 2H), 2.35 (s, 3H), 2.04 (br, 1H), 1.89 - 1.80 (m, 1H), $1.76-1.67(\mathrm{~m}, 1 \mathrm{H}), 1.52-1.44(\mathrm{~m}, 2 \mathrm{H}), 1.42(\mathrm{~s}, 9 \mathrm{H}) .{ }^{19} \mathrm{~F}$ NMR $\left(376 \mathrm{MHz}, \mathrm{CDCl}_{3}\right) \delta-117.3(\mathrm{~s}$, 2F). ${ }^{13} \mathrm{C}$ NMR $\left(100 \mathrm{MHz}, \mathrm{CDCl}_{3}\right) \delta 154.7,138.5,133.8,129.0,127.6,122.8\left(\mathrm{t}, \mathrm{C}-\mathrm{F}, 1 J_{\mathrm{C}-\mathrm{F}}=247.0 \mathrm{~Hz}\right)$, 79.6, $73.4\left(\mathrm{t}, \mathrm{C}-\mathrm{F}, 2 J_{C-F}=28.8 \mathrm{~Hz}\right), 42.8(\mathrm{br}), 39.3\left(\mathrm{t}, \mathrm{C}-\mathrm{F}, 2 J_{C-F}=23.2 \mathrm{~Hz}\right), 28.3,24.9,24.3,21.1 . \mathrm{MS}$ (ESI): m/z (\%) $300(100), 378\left([\mathrm{M}+\mathrm{Na}]^{+}\right)$. HRMS (ESI-quadrupole) $\mathrm{m} / \mathrm{z}:[\mathrm{M}+\mathrm{Na}]^{+}$Calcd for $\mathrm{C}_{19} \mathrm{H}_{27} \mathrm{~F}_{2} \mathrm{NO}_{3} \mathrm{Na} 378.1857$; Found 378.1848. 


\section{References}

(1) Fu, M.-C.; Shang, R.; Zhao, B.; Wang, B.; Fu, Y. Photocatalytic Decarboxylative Alkylations Mediated by Triphenylphosphine and Sodium Iodide. Science 2019, 363, 1429-1434.

(2) Wang, J.; Shang, M.; Lundberg, H.; Feu, K. S.; Hecker, S. J.; Qin, T.; Blackmond, D. G.; Baran, P. S. Cu-Catalyzed Decarboxylative Borylation. ACS Catal. 2018, 8, 9537-9542.

(3) Qin, T.; Cornella, J.; Li, C.; Malins, L. R.; Edwards, J. T.; Kawamura, S.; Maxwell, B. D.; Eastgate, M. D.; Baran, P. S. A General Alkyl-Alkyl Cross-Coupling Enabled by Redox-Active Esters and Alkylzinc Reagents. Science 2016, 352, 801-805.

(4) He, J.-Y.; Chen, G-L.; Zhang, B-X.; Li, Y.; Chen, J-R.; Xiao, W-J.; Liu, F.; Li, C. Catalytic Decarboxylative Radical Sulfonylation. Chem. 2020, 6, 1149-1159.

(5) Gao, X.; Cheng, R.; Xiao, Y.-L.; Wan, X.-L.; Zhang, X..Copper-Catalyzed Highly Enantioselective Difluoroalkylation of Secondary Propargyl Sulfonates with Difluoroenoxysilanes. Chem 2019, 5, 2987-2999. 
8. Copies of Spectra of Compounds 1, 3, 5-7.

\section{1,3-Dioxoisoindolin-2-yl 2-ethylhexanoate (1e)}
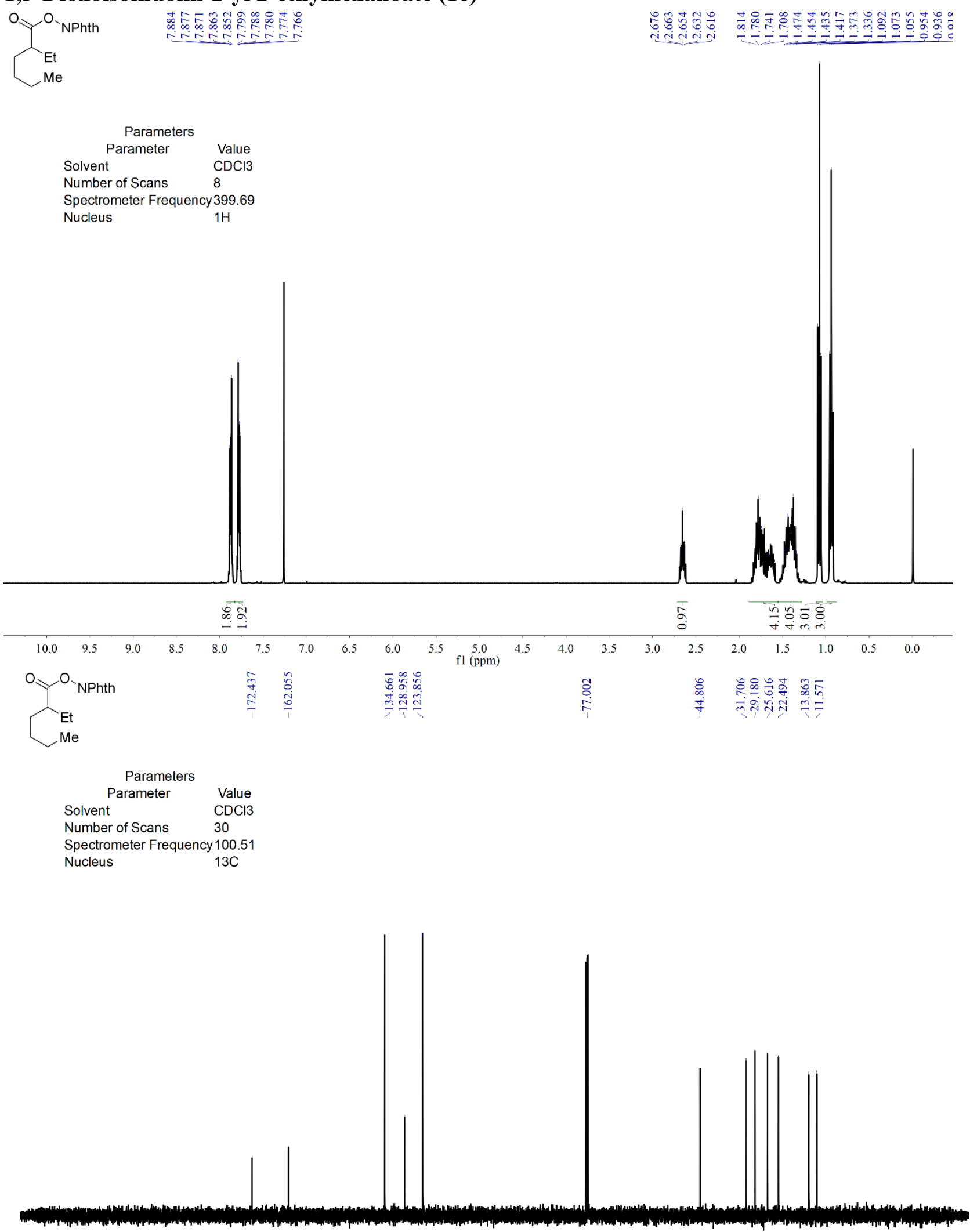

$\begin{array}{lllllllllllllllllllllllllllllllllll}230 & 220 & 210 & 200 & 190 & 180 & 170 & 160 & 150 & 140 & 130 & 120 & 110 & 100 & 90 & 80 & 70 & 60 & 50 & 40 & 30 & 20 & 10 & 0 & -10 & -20 & -3\end{array}$ 


\section{1,3-Dioxoisoindolin-2-yl propionate (1k)}

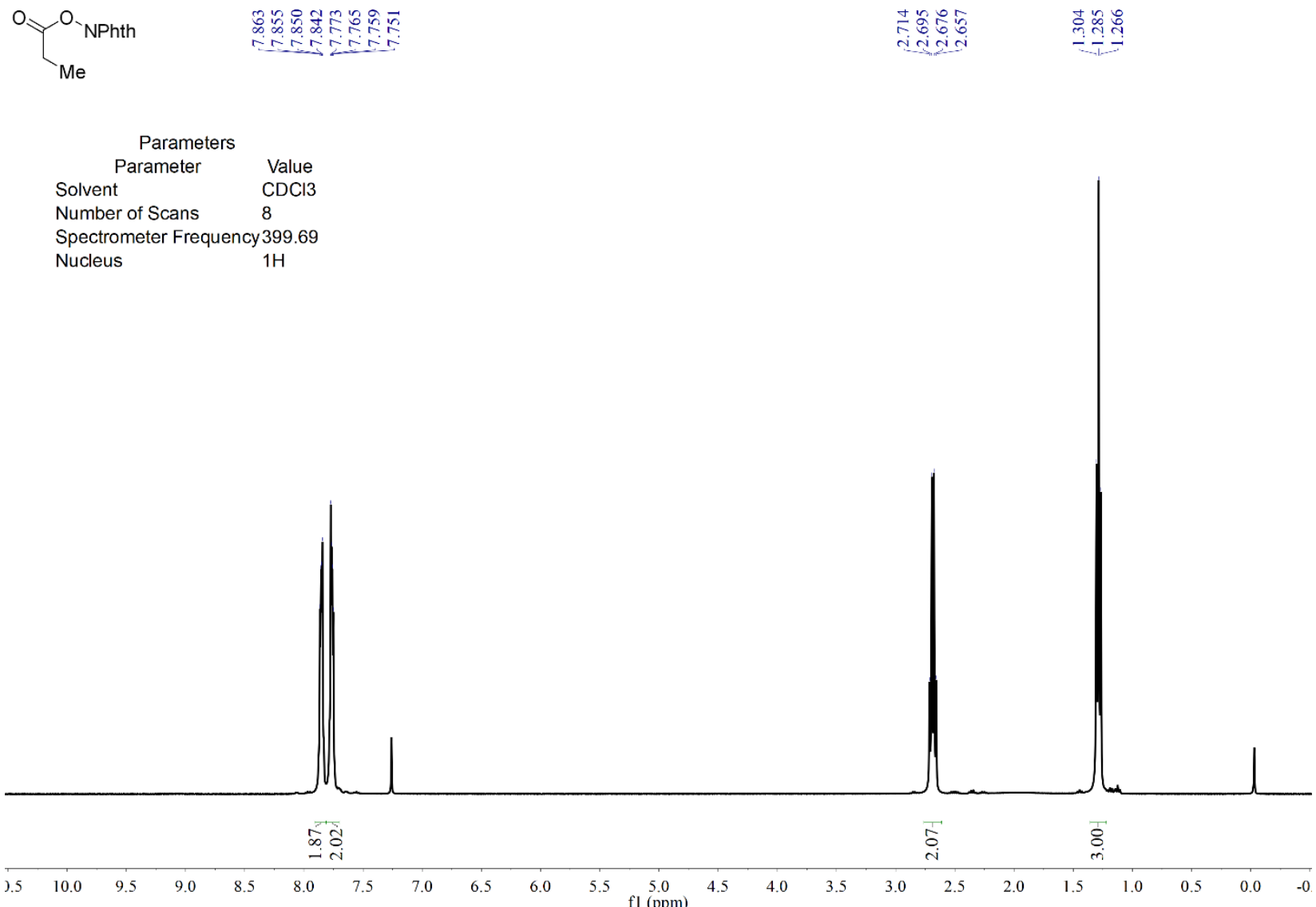

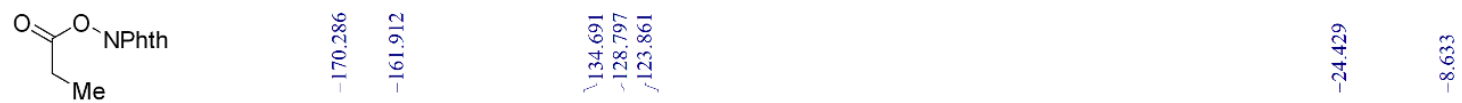

\begin{tabular}{ll}
\multicolumn{2}{c}{ Parameters } \\
Parameter & Value \\
Solvent & $\mathrm{CDCl} 3$ \\
Number of Scans & 128 \\
Spectrometer Frequency 125.77 \\
Nucleus & $13 \mathrm{C}$
\end{tabular}

$\begin{array}{llllllllllll}215 & 205 & 195 & 185 & 175 & 165 & 155 & 145 & 135 & 125 & 115 & 105 \\ \text { fl (ppm) }\end{array}$ 
1,3-Dioxoisoindolin-2-yl 3-(1H-indol-3-yl)propanoate (1I)
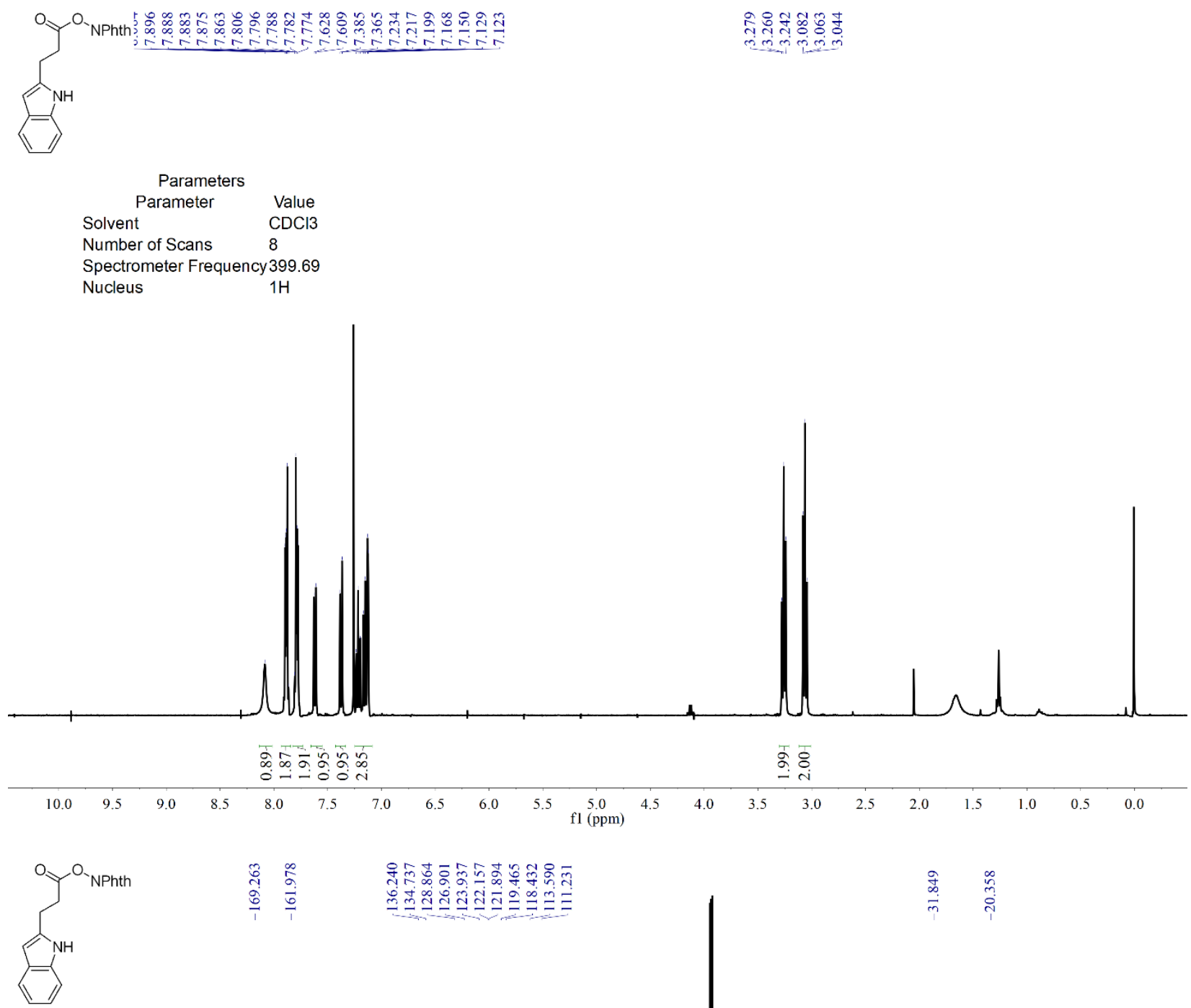

\section{$\begin{array}{ll}\hat{8} & \infty \\ \text { à } & 5 \\ 0 & 0\end{array}$

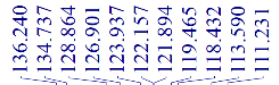

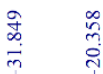

\begin{tabular}{lc}
\multicolumn{1}{c}{ Parameters } \\
Parameter & Value \\
Solvent & CDCl3 \\
Number of Scans & 128 \\
Spectrometer Frequency 125.77 \\
Nucleus & $13 \mathrm{C}$
\end{tabular}

$\begin{array}{llllllllllll}215 & 205 & 195 & 185 & 175 & 165 & 155 & 145 & 135 & 125 & 115 & \begin{array}{c}105 \\ \mathrm{fl}(\mathrm{ppm})\end{array}\end{array}$ 
1,3-Dioxoisoindolin-2-yl 2-phenylcyclopropane-1-carboxylate (( \pm$)$-cis-10).
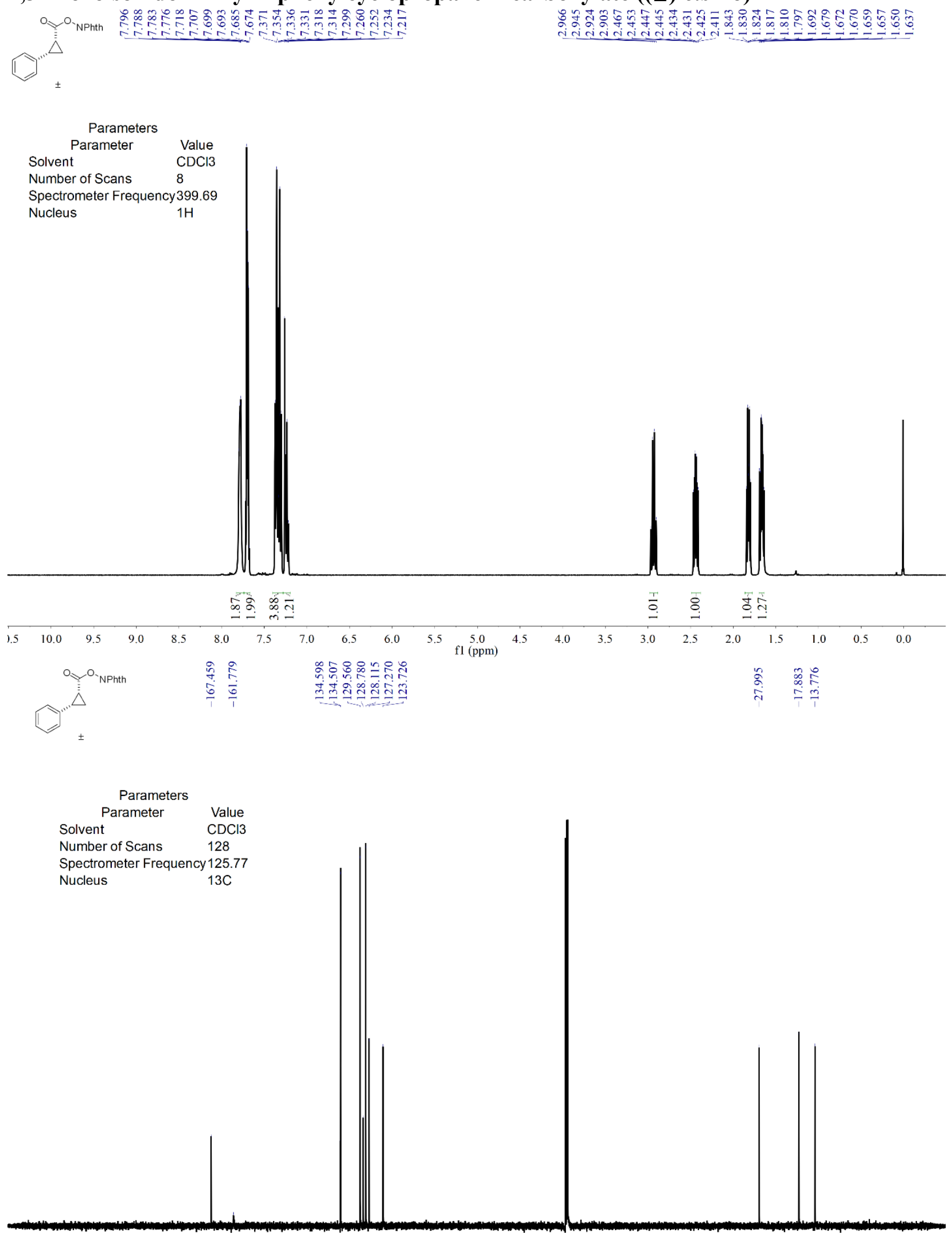

$\begin{array}{llllllllllll}215 & 205 & 195 & 185 & 175 & 165 & 155 & 145 & 135 & 125 & 115 & \begin{array}{c}105 \\ \text { fl (ppm) }\end{array}\end{array}$ 
1,3-Dioxoisoindolin-2-yl ((benzyloxy)carbonyl)-L-phenylalanyl-D-leucinate (1s)
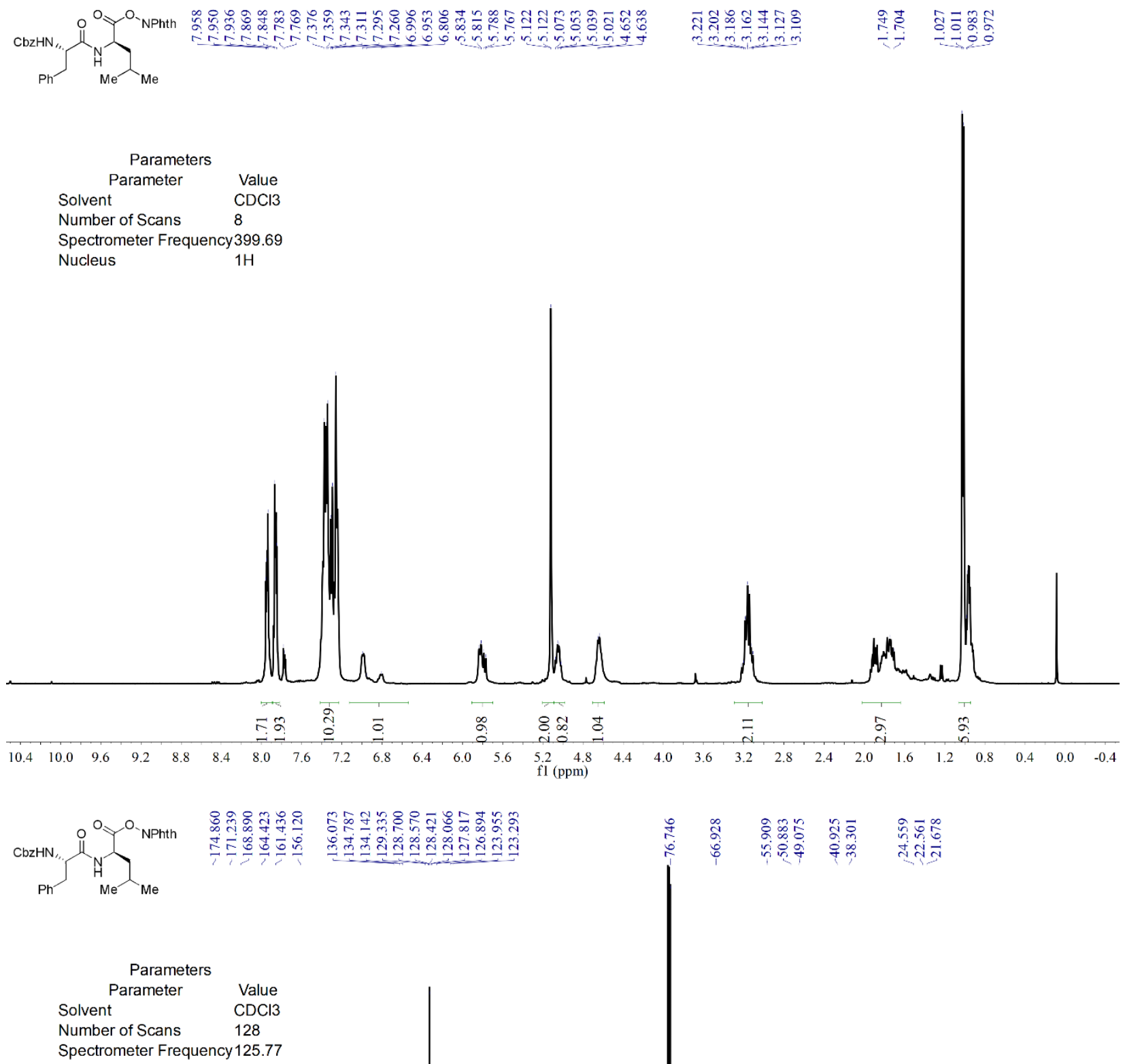

Spectrometer Frequency 125.77

Nucleus

$13 \mathrm{C}$

\begin{tabular}{llllllllllll}
\hline 215 & 205 & 195 & 185 & 175 & 165 & 155 & 145 & 135 & 125 & 115 & $\begin{array}{c}105 \\
\text { fl (ppm) }\end{array}$
\end{tabular} 
Tert-butyl 4-(1,1-difluoro-2-oxo-2-(p-tolyl)ethyl)piperidine-1-carboxylate (3a)<smiles>O=C(OC(=O)C(F)(F)C1CCN(C(=O)O)CC1)c1ccccc1</smiles>

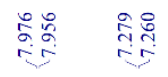

$\stackrel{n}{\stackrel{*}{*}}$

\begin{tabular}{lc}
\multicolumn{2}{c}{ Parameters } \\
\multicolumn{1}{c}{ Parameter } & Value \\
Solvent & CDCl3 \\
Number of Scans & 8 \\
Spectrometer Frequency 399.69 \\
Nucleus & $1 \mathrm{H}$
\end{tabular}

Nucleus

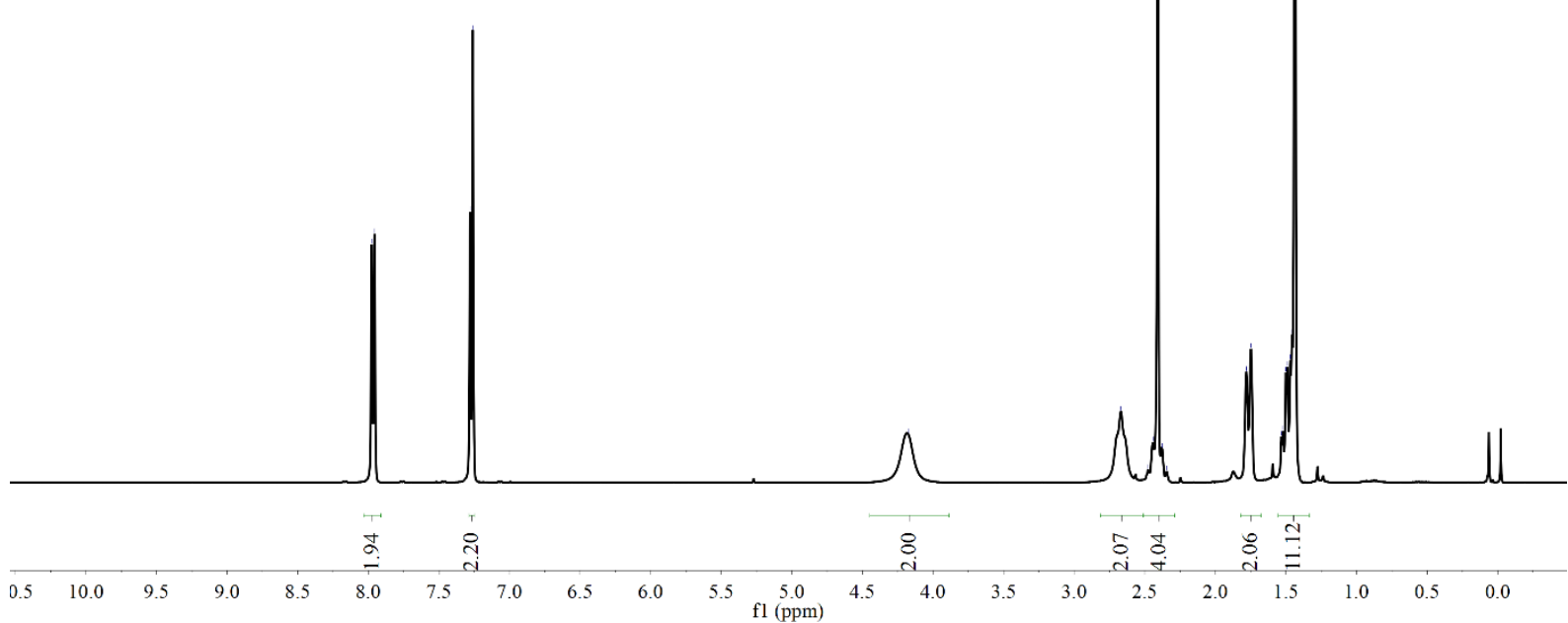<smiles>O=C(O)C(=O)N1CCC(C(F)(F)C(=O)OC(=O)c2ccccc2)CC1</smiles>

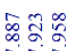

훙호

Boc

$$
\begin{array}{ll}
\multicolumn{1}{c}{\text { Parameters }} \\
\text { Parameter } & \multicolumn{1}{c}{\text { Value }} \\
\text { Solvent } & \text { CDCl3 } \\
\text { Number of Scans } & 16 \\
\text { Spectrometer Frequency } 376.05 \\
\text { Nucleus } & 19 \mathrm{~F}
\end{array}
$$

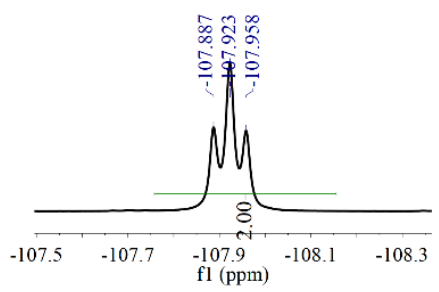

\&

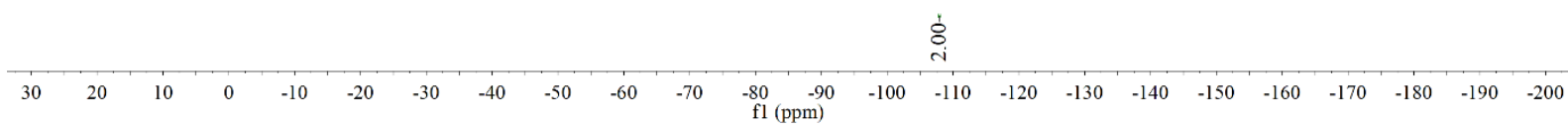



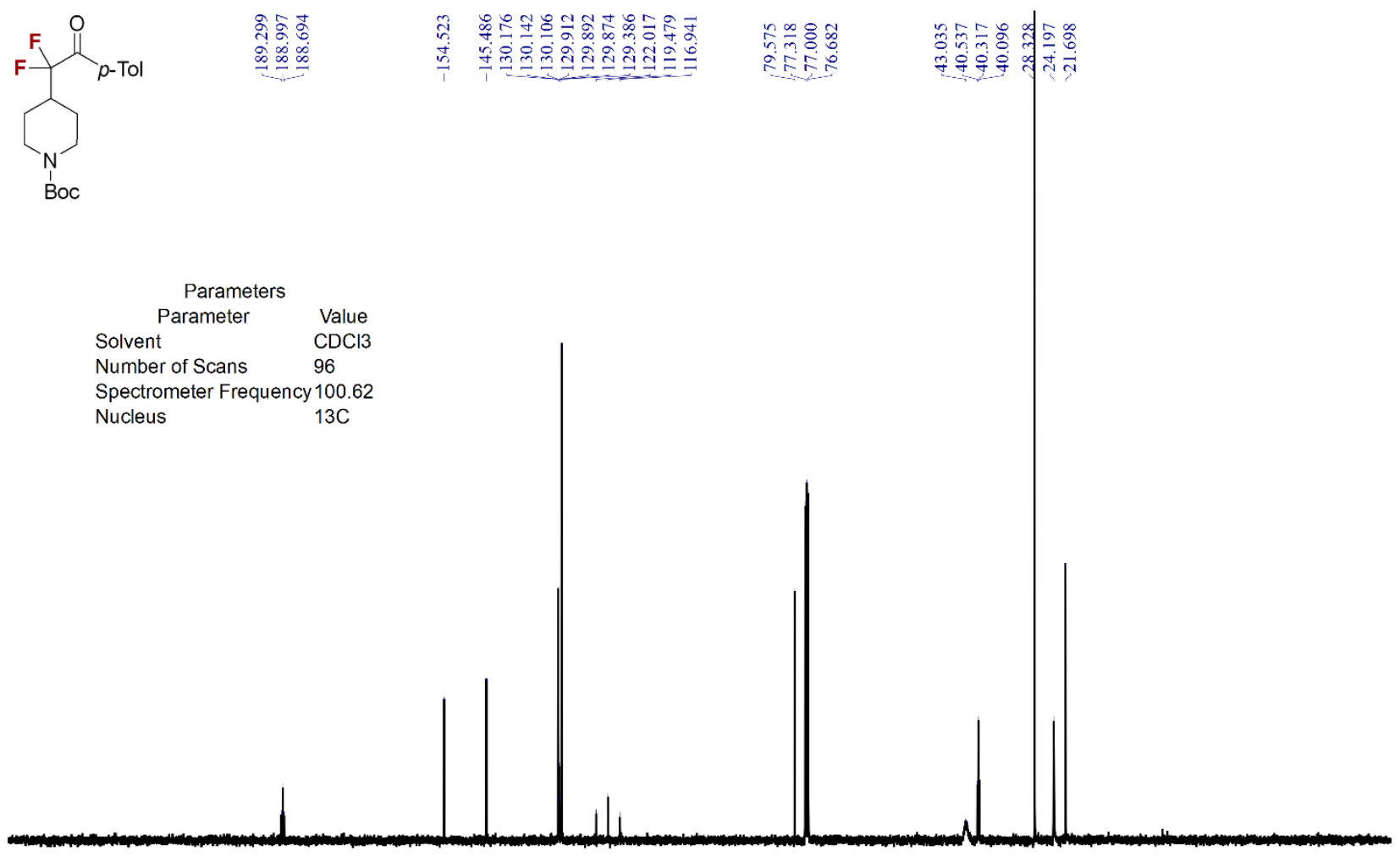

\begin{tabular}{llllllllllllllllllllllllllllllllllllllll}
\hline 245 & 235 & 225 & 215 & 205 & 195 & 185 & 175 & 165 & 155 & 145 & 135 & 125 & 115 & 105 & 95 & 85 & 75 & 65 & 55 & 45 & 35 & 25 & 15 & 5 & -5 & -15 & -25 & -35 & -45
\end{tabular}

\section{2,2-Difluoro-2-(tetrahydro-2H-pyran-4-yl)-1-(p-tolyl)ethan-1-one (3b)}
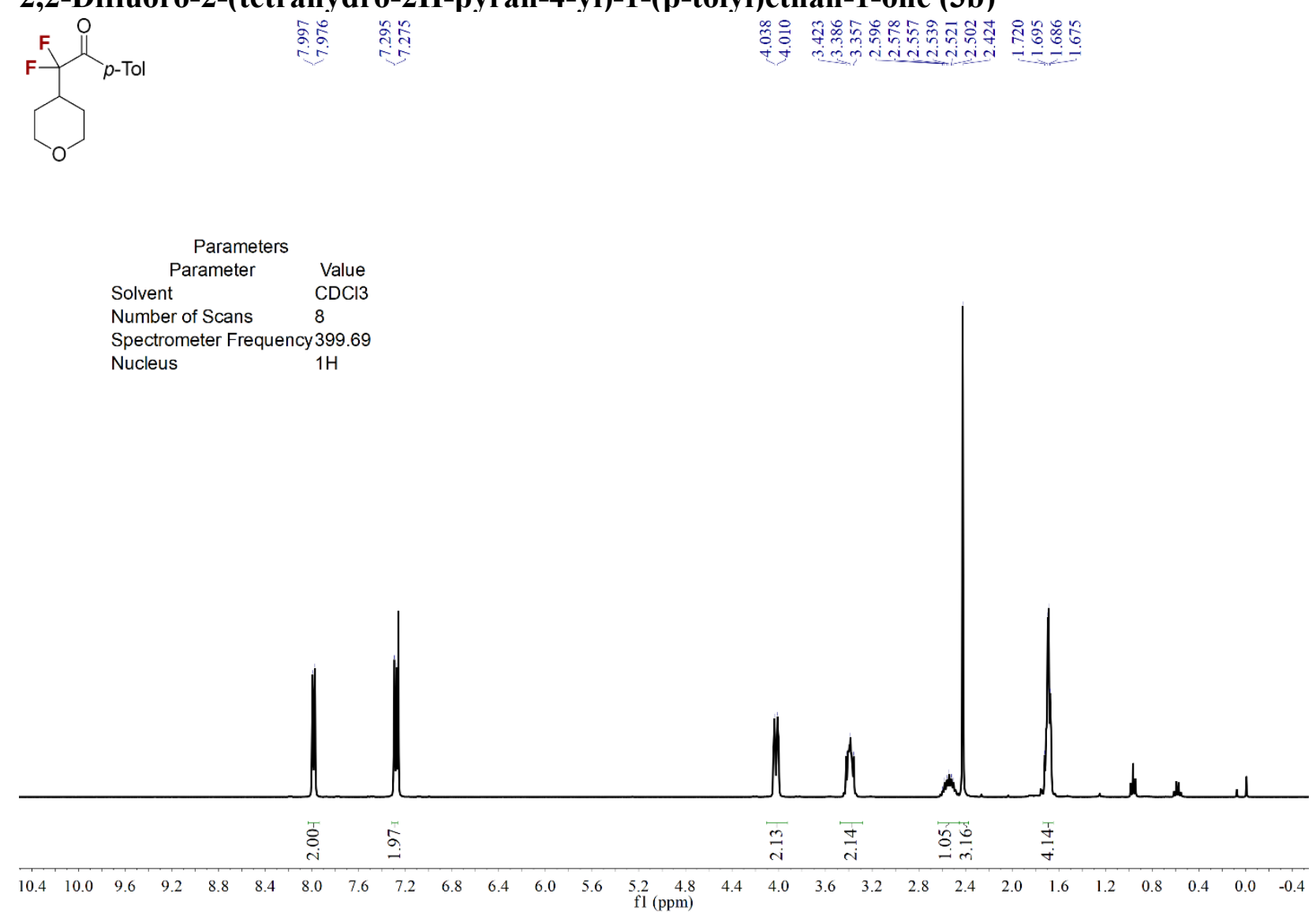
$\overbrace{0}^{p-\mathrm{Tol}}$

$$
\begin{array}{ll}
\quad \text { Parameter } & \text { Value } \\
\text { Solvent } & \text { CDCl3 } \\
\text { Number of Scans } & 16 \\
\text { Spectrometer Frequency } 376.05 \\
\text { Nucleus } & 19 \mathrm{~F}
\end{array}
$$
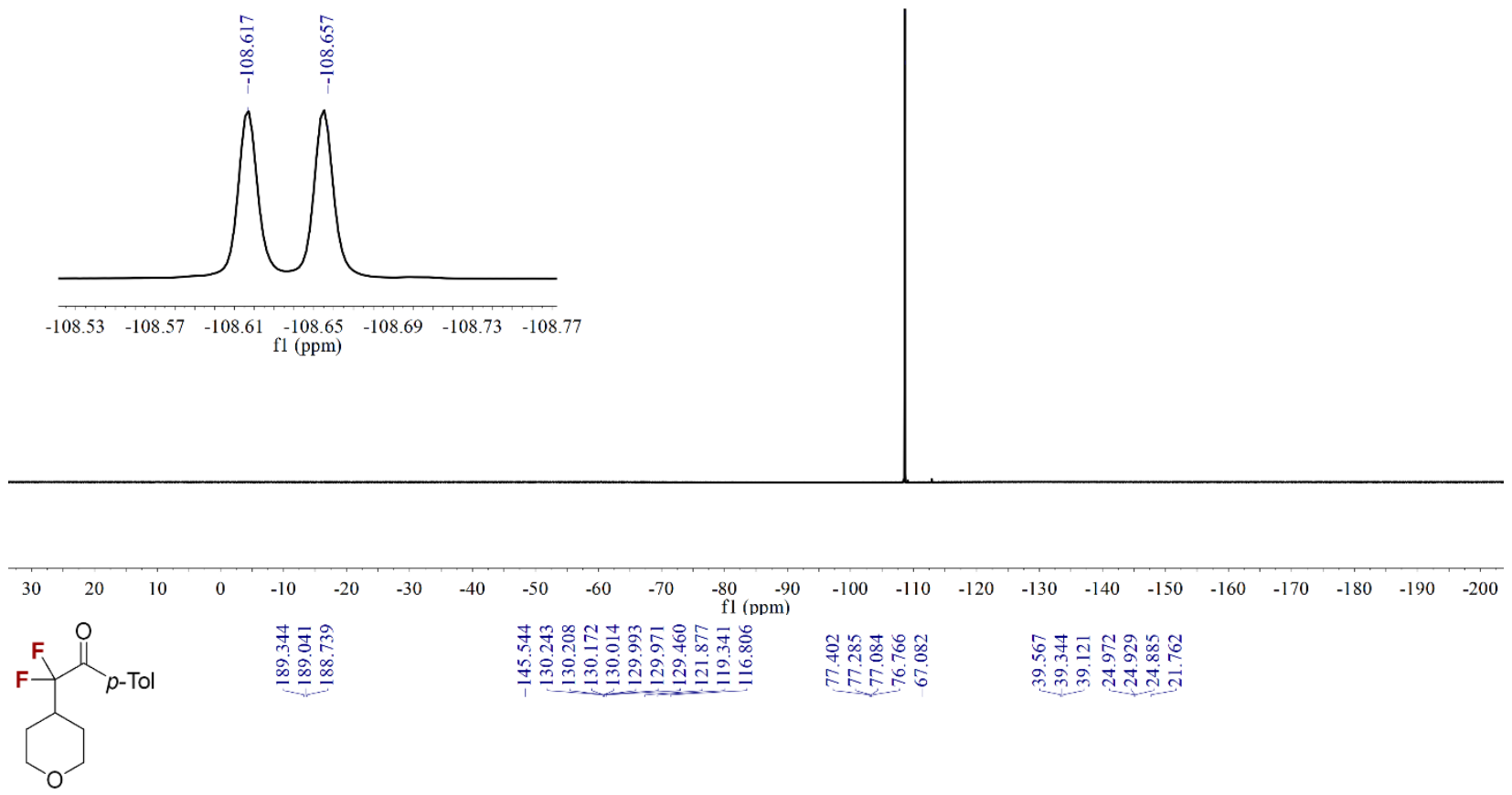

\begin{tabular}{ll}
\multicolumn{1}{c}{$\begin{array}{c}\text { Parameters } \\
\text { Parameter }\end{array}$} & Value \\
Solvent & $\mathrm{CDCl} 3$ \\
Number of Scans & 44 \\
Spectrometer Frequency 100.62 \\
Nucleus
\end{tabular}

Nucleus

$13 \mathrm{C}$ 
2-Cyclohexyl-2,2-difluoro-1-(p-tolyl)ethan-1-one (3c)<smiles>O=C(O[Ga])C(F)(F)C1CCCCC1</smiles>

ลूล ํํำ

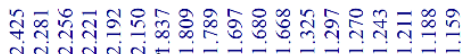

Parameters

Parameter Value

Solvent $\quad \mathrm{CDCl} 3$

Number of Scans 8

Spectrometer Frequency 399.69

Nucleus

$1 \mathrm{H}$
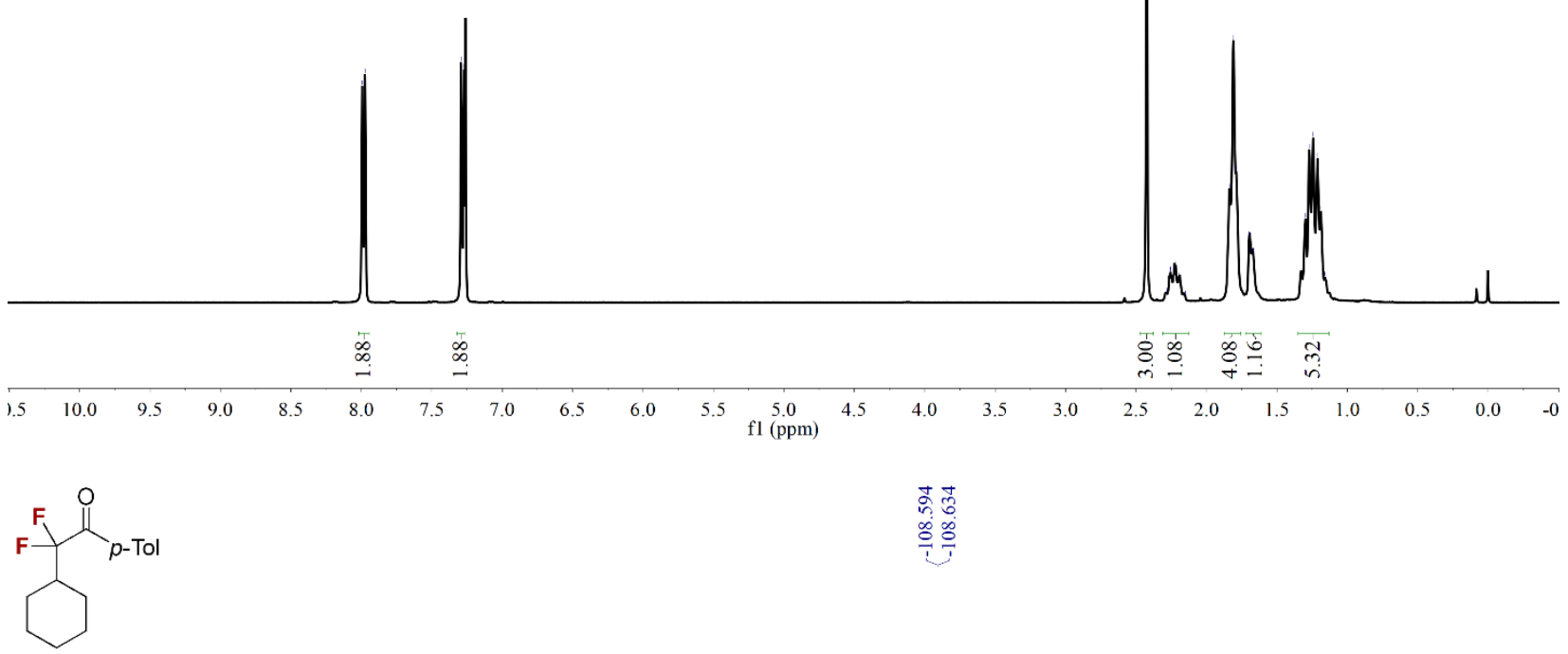

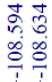
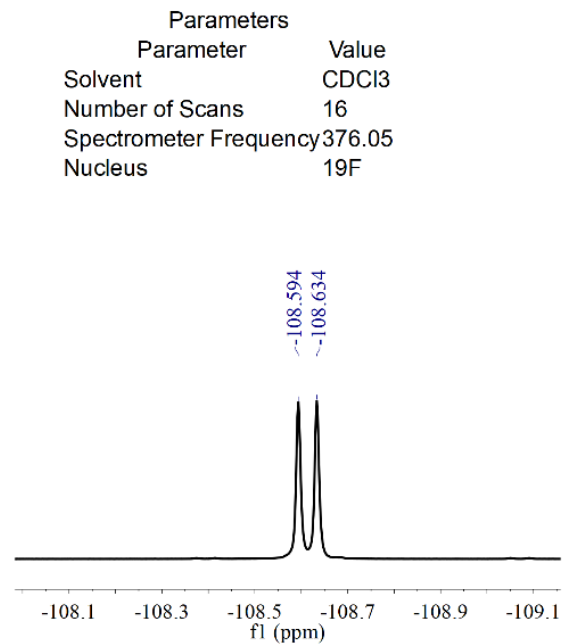

\begin{tabular}{llllllllllllllllllllllllllll}
\hline 30 & 20 & 10 & 0 & -10 & -20 & -30 & -40 & -50 & -60 & -70 & -80 & -90 & -100 & -110 & -120 & -130 & -140 & -150 & -160 & -170 & -180 & -190 & -200
\end{tabular} 


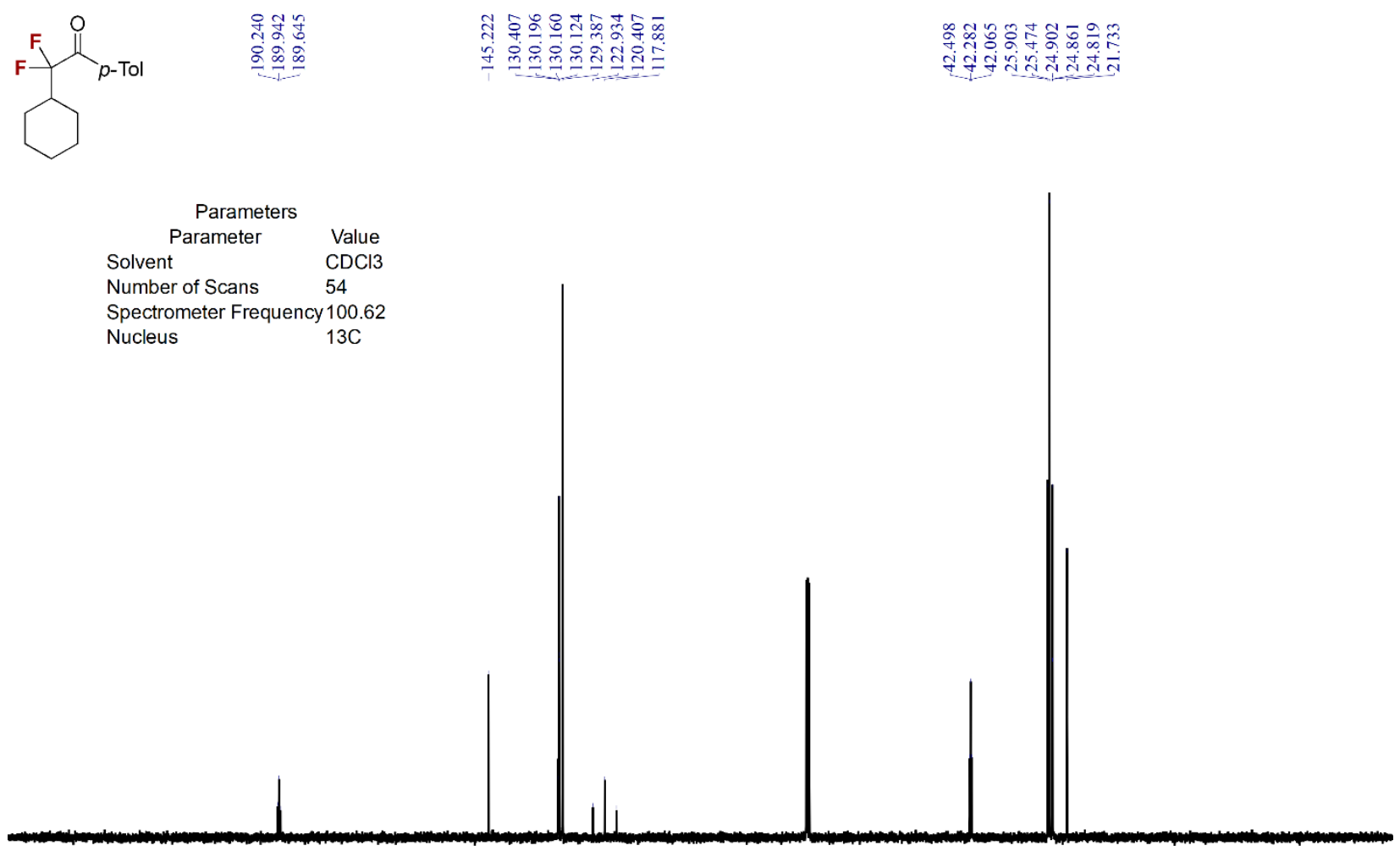

$\begin{array}{llllllllllllllllllllllllllllllllllllll}245 & 235 & 225 & 215 & 205 & 195 & 185 & 175 & 165 & 155 & 145 & 135 & 125 & 115 & \begin{array}{l}105 \\ \mathrm{fl}(\mathrm{ppm})\end{array} & 85 & 75 & 65 & 55 & 45 & 35 & 25 & 15 & 5 & -5 & -15 & -25 & -35 & -45\end{array}$

Tert-butyl 3-(1,1-difluoro-2-oxo-2-(p-tolyl)ethyl)azetidine-1-carboxylate (3d)<smiles>O=C([O-])N1CC(C(F)(F)C(=O)O[Na])C1</smiles>

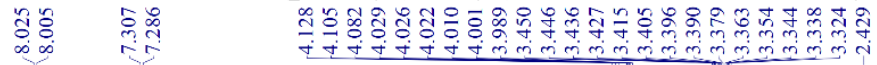

\begin{tabular}{ll}
\multicolumn{1}{c}{ Parameters } \\
Parameter & Value \\
Solvent & $\mathrm{CDCl} 3$ \\
Number of Scans & 8 \\
Spectrometer Frequency 399.69 \\
Nucleus & $1 \mathrm{H}$
\end{tabular}

Nucleus

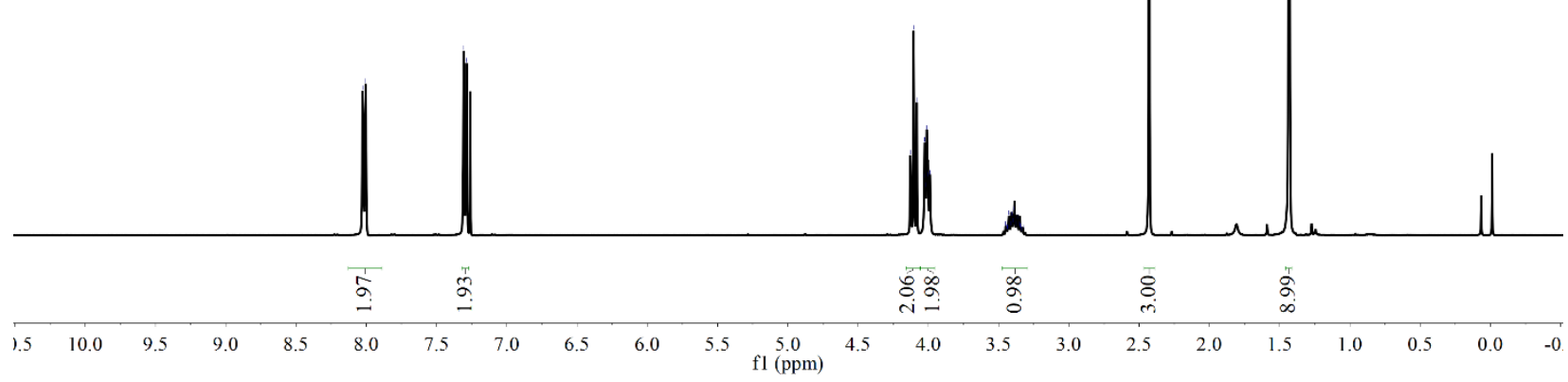




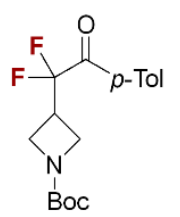

Boc

\begin{tabular}{ll}
\multicolumn{1}{c}{ Parameters } \\
Parameter & Value \\
Solvent & $\mathrm{CDCl} 3$ \\
Number of Scans & 16 \\
Spectrometer Frequency 376.05 \\
Nucleus & $19 \mathrm{~F}$
\end{tabular}
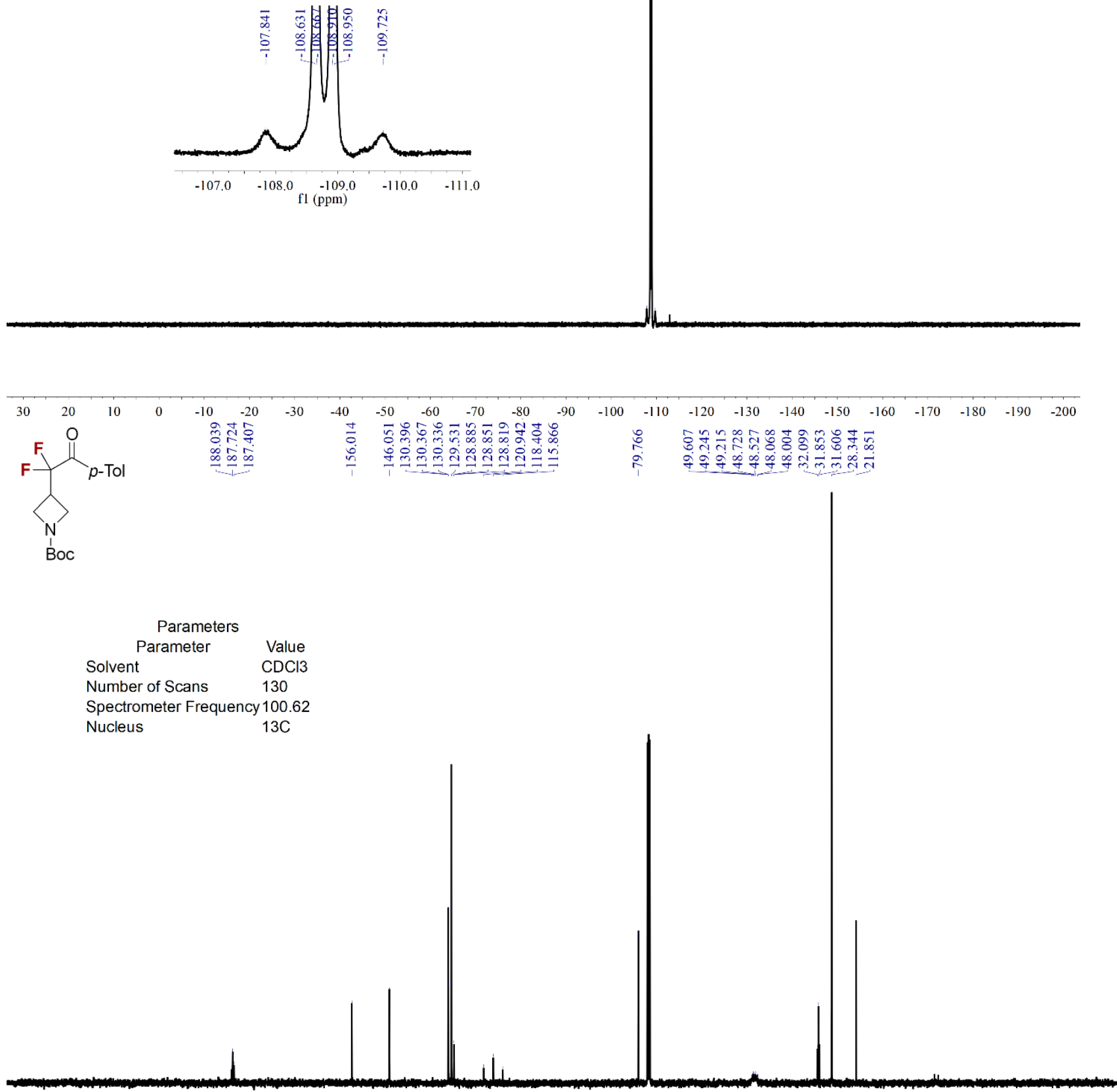

$\begin{array}{lllllllllllllllllllllllllllllllllll}245 & 235 & 225 & 215 & 205 & 195 & 185 & 175 & 165 & 155 & 145 & 135 & 125 & 115 & 105 & 95 & 85 & 75 & 65 & 55 & 45 & 35 & 25 & 15 & 5 & -5 & -15 & -25 & -35 & -45\end{array}$ $\mathrm{fl}(\mathrm{ppm})$ 


\section{3-Ethyl-2,2-difluoro-1-(p-tolyl)heptan-1-one (3e)}

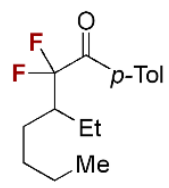

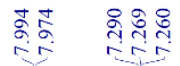

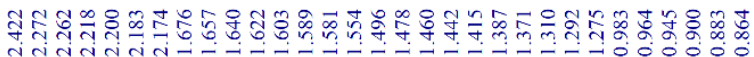

\begin{tabular}{ll}
\multicolumn{1}{c}{ Parameters } \\
Parameter & Value \\
Solvent & CDCl3 \\
Number of Scans & 8 \\
Spectrometer Frequency 399.69 \\
Nucleus & $1 \mathrm{H}$
\end{tabular}

Nucleus

$1 \mathrm{H}$

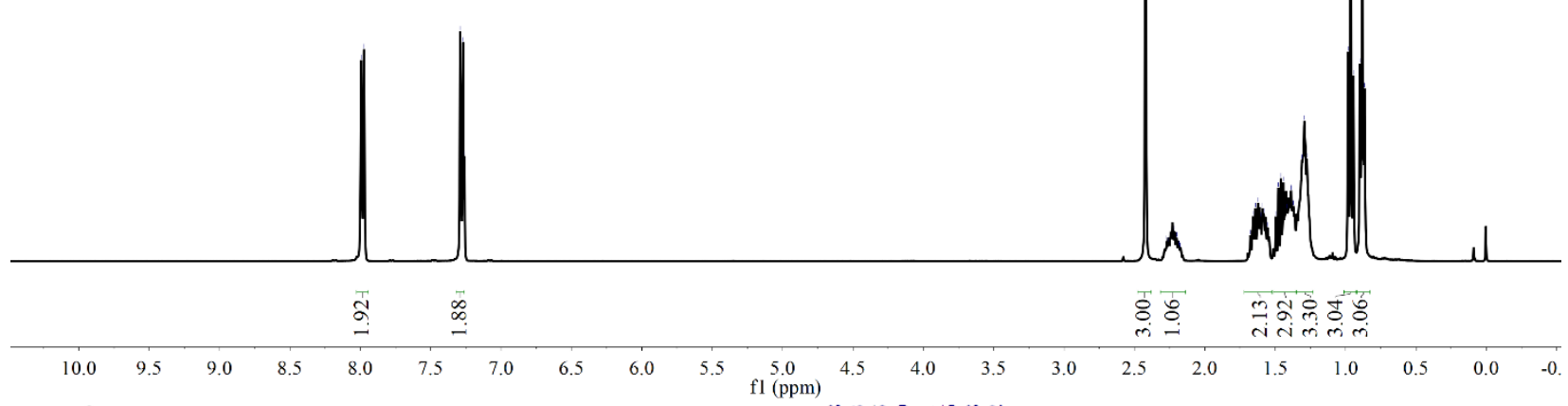<smiles>CCCCC(CC)C(F)(F)C(=O)O[Ga]</smiles>

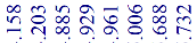

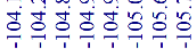

Me

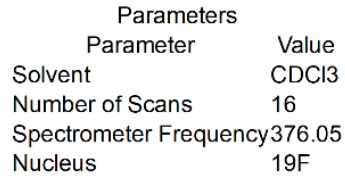

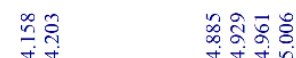

응

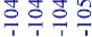

$\stackrel{\infty}{\infty}$

nis

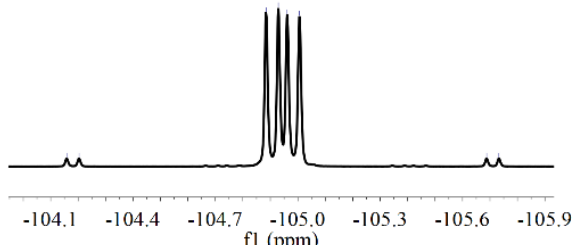

$\begin{array}{llllllllllll}30 & 20 & 10 & 0 & -10 & -20 & -30 & -40 & -50 & -60 & -70 & -80 \begin{array}{r}-90 \\ \text { fl (ppm) }\end{array}\end{array}$ 


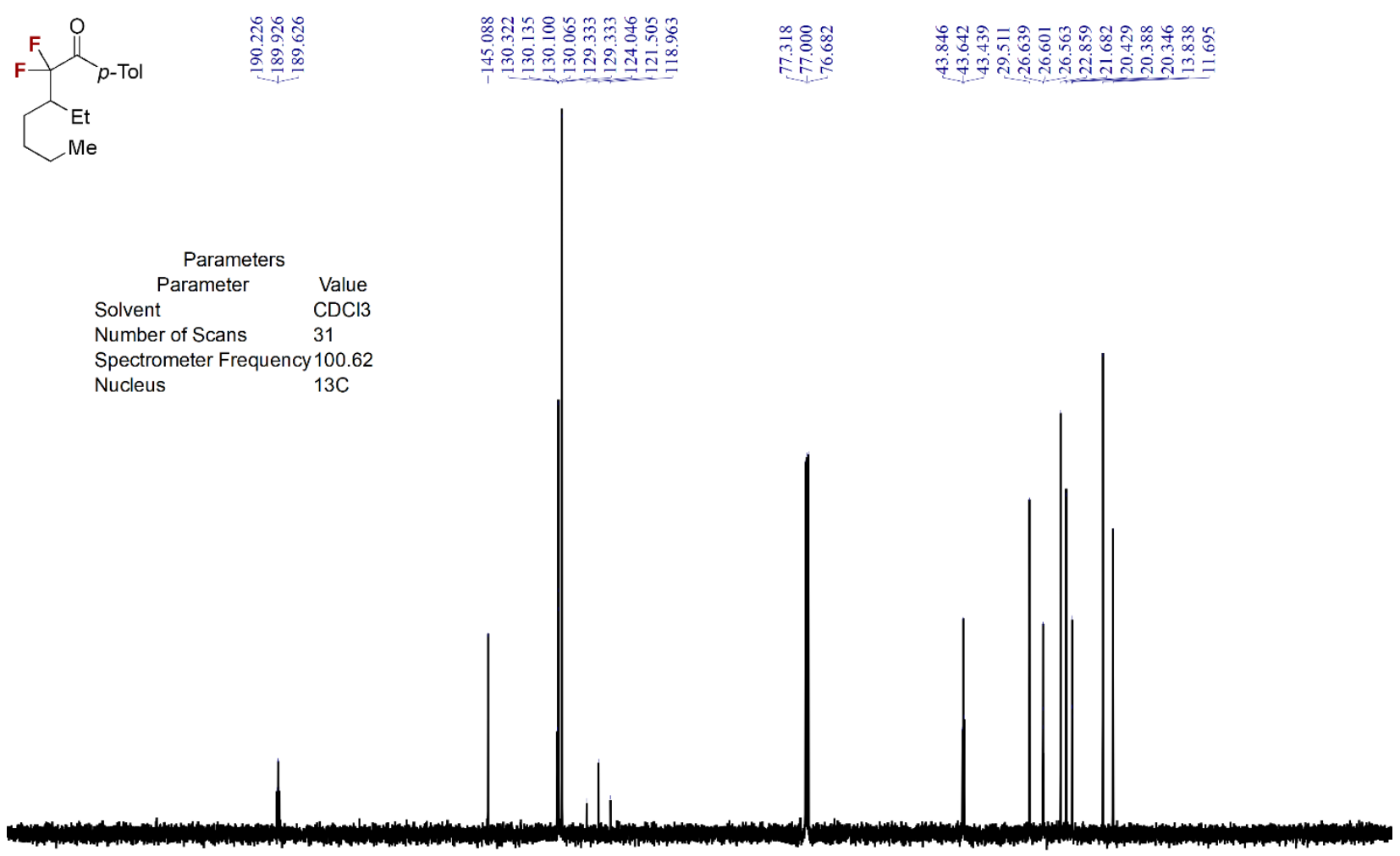

$\begin{array}{llllllllllllllllllllllllllllllllllllll}245 & 235 & 225 & 215 & 205 & 195 & 185 & 175 & 165 & 155 & 145 & 135 & 125 & 115 & \begin{array}{c}105 \\ \mathrm{fl}(\mathrm{ppm})\end{array} & 85 & 75 & 65 & 55 & 45 & 35 & 25 & 15 & 5 & -5 & -15 & -25 & -35 & -45\end{array}$

Tert-butyl (3,3-difluoro-4-oxo-1-phenyl-4-(p-tolyl)butan-2-yl)carbamate (3f)

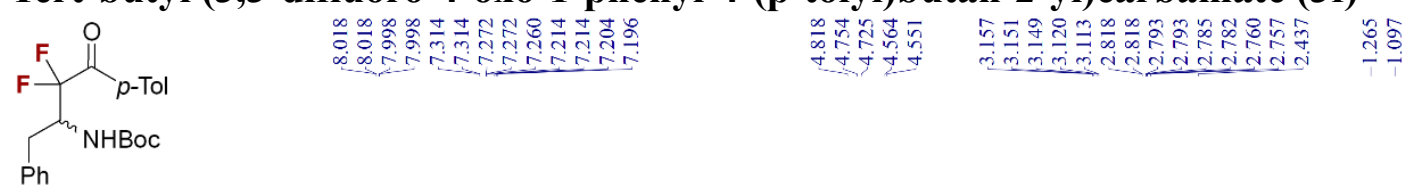

\begin{tabular}{ll}
\multicolumn{2}{c}{ Parameters } \\
Parameter & Value \\
Solvent & CDCl3 \\
Number of Scans & 8 \\
Spectrometer Frequency 399.69 \\
Nucleus & $1 \mathrm{H}$
\end{tabular}

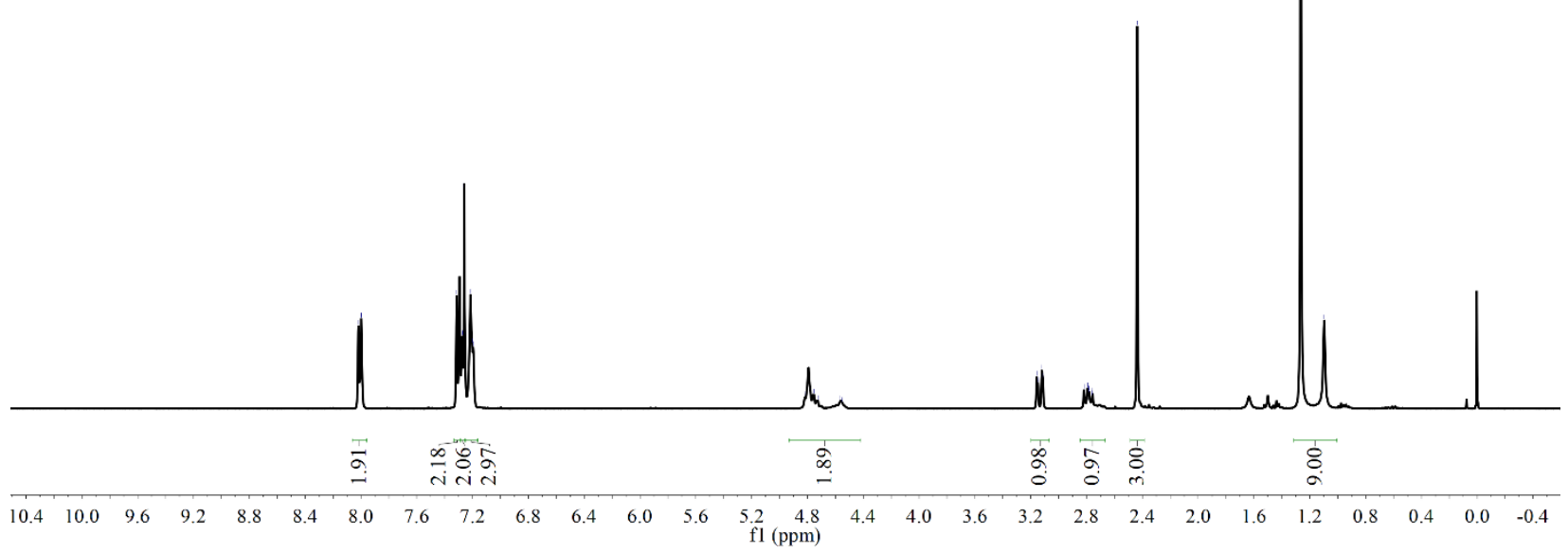


$\int_{\mathrm{Ph}}^{\mathrm{NHBOC}}$

\begin{tabular}{ll}
\multicolumn{2}{c}{ Parameters } \\
Parameter & Value \\
Solvent & $\mathrm{CDCl} 3$ \\
Number of Scans & 16 \\
Spectrometer Frequency 376.05 \\
Nucleus & $19 \mathrm{~F}$
\end{tabular}

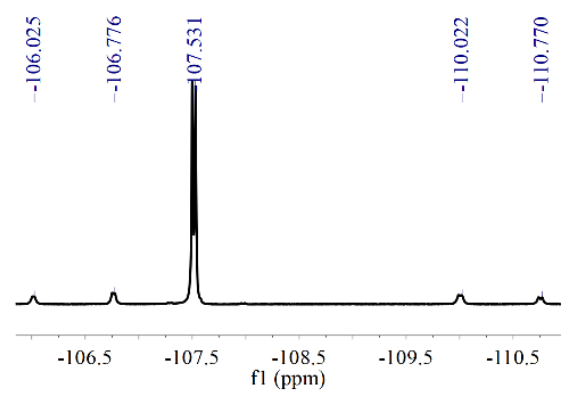

\begin{tabular}{lllllllllllllllllllllllllllll}
\hline 30 & 20 & 10 & 0 & -10 & -20 & -30 & -40 & -50 & -60 & -70 & -80 & -90 & -100 & -110 & -120 & -130 & -140 & -150 & -160 & -170 & -180 & -190 & -200
\end{tabular}

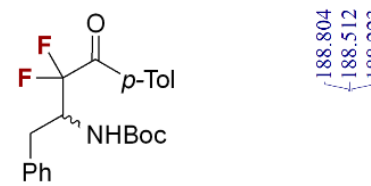

$\mathrm{Ph}$

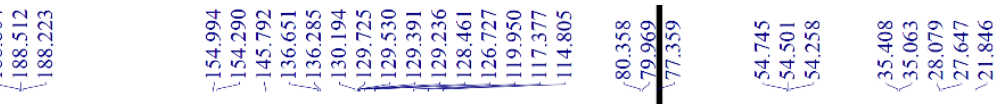

Parameters
Parameter

Solvent

Spectrometer Frequency 100.62

Nucleus

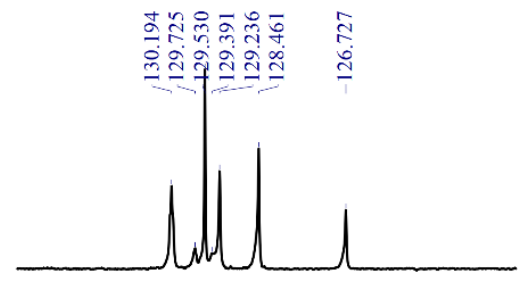

$\begin{array}{llllllllll}133 & 132 & 131 & 130 & 129 & 128 & 127 & 126 & 125 & 124\end{array}$ 


\section{2,2-Difluoro-3,3-dimethyl-1-(p-tolyl)butan-1-one (3g)}

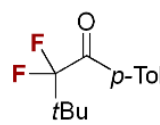

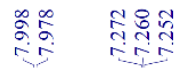

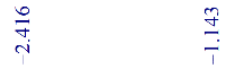

tBu

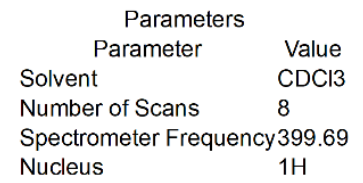

Nucleus
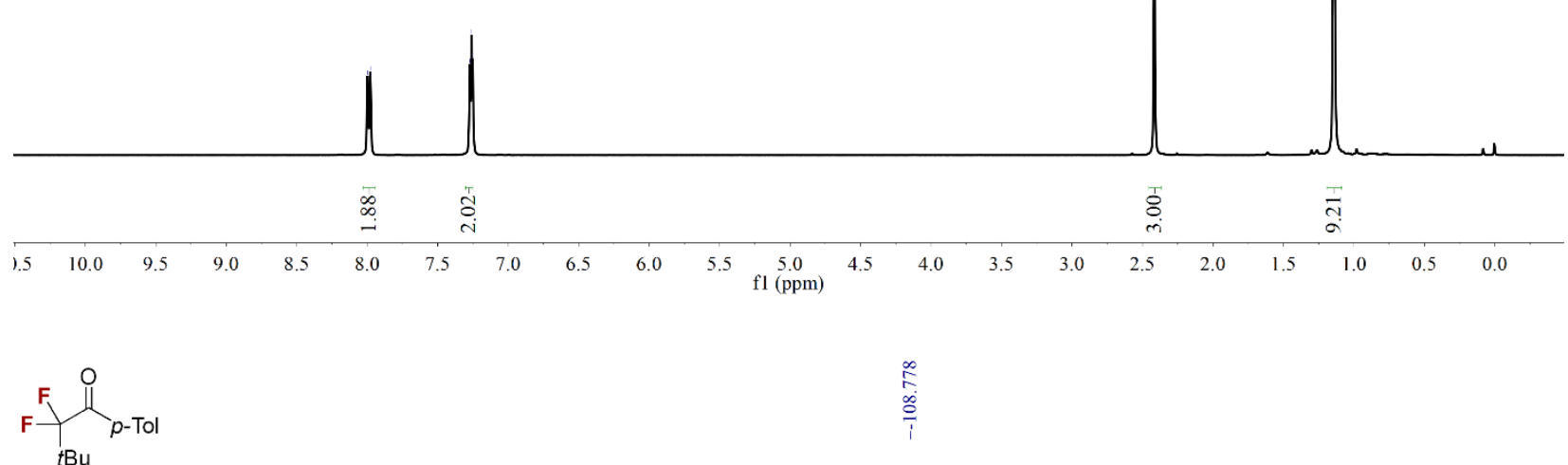

$t \mathrm{Bu}$

\begin{tabular}{ll}
\multicolumn{3}{c}{ Parameters } \\
Parameter & Value \\
Solvent & CDCl3 \\
Number of Scans & 16 \\
Spectrometer Frequency & 376.05 \\
Nucleus & $19 F$
\end{tabular}

\begin{tabular}{lllllllllllllllllllllllllllllll}
\hline 30 & 20 & 10 & 0 & -10 & -20 & -30 & -40 & -50 & -60 & -70 & -80 & -90 & -100 & -110 & -120 & -130 & -140 & -150 & -160 & -170 & -180 & -190 & -200
\end{tabular} 

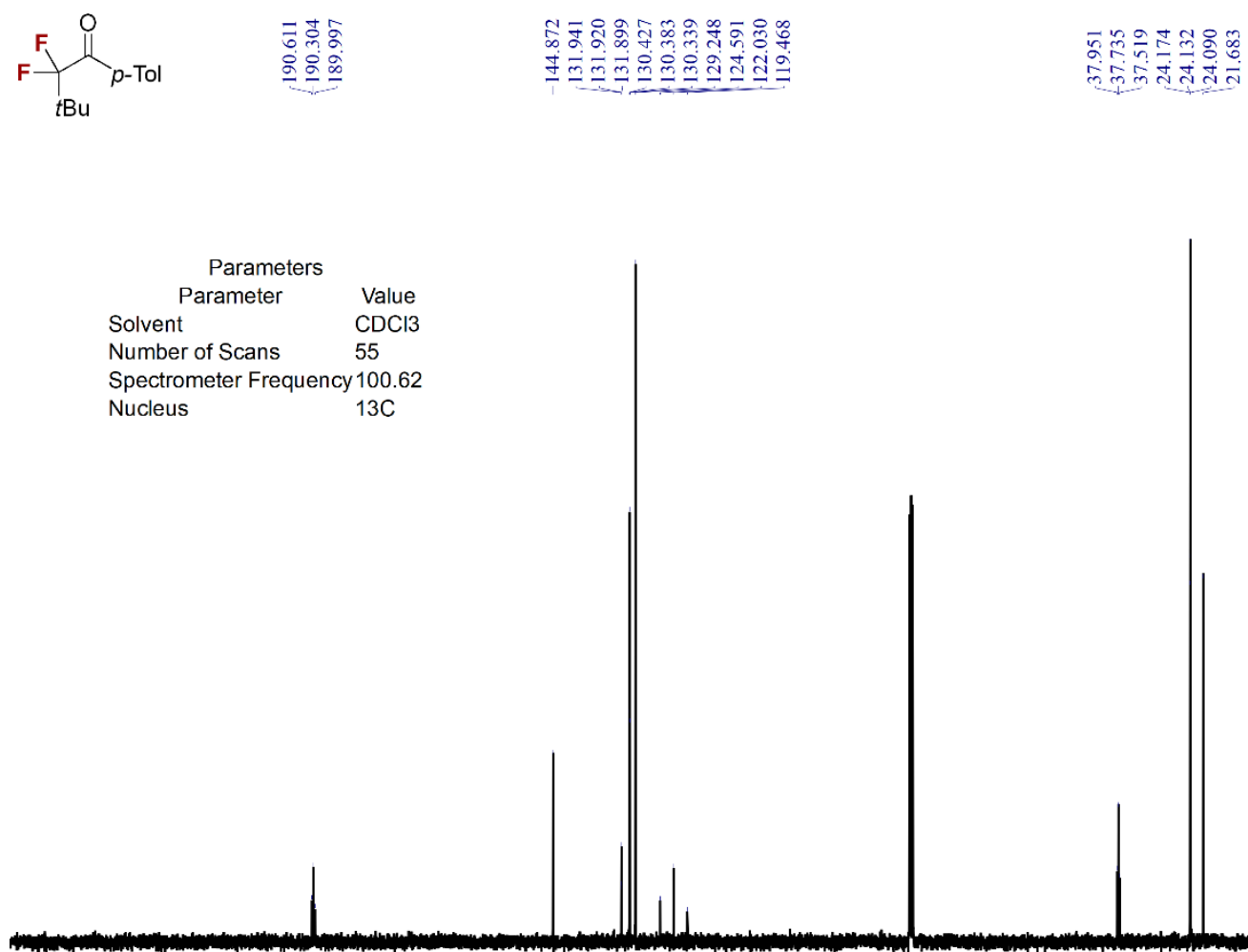

$\begin{array}{lllllllllllllllllllllllllllllll}245 & 235 & 225 & 215 & 205 & 195 & 185 & 175 & 165 & 155 & 145 & 135 & 125 & 115 & 105 & 95 & 85 & 75 & 65 & 55 & 45 & 35 & 25 & 15 & 5 & -5 & -15 & -25 & -35 & -45\end{array}$

\section{2,2-Difluoro-3,3-dimethyl-1-(p-tolyl)pentan-1-one (3h)}
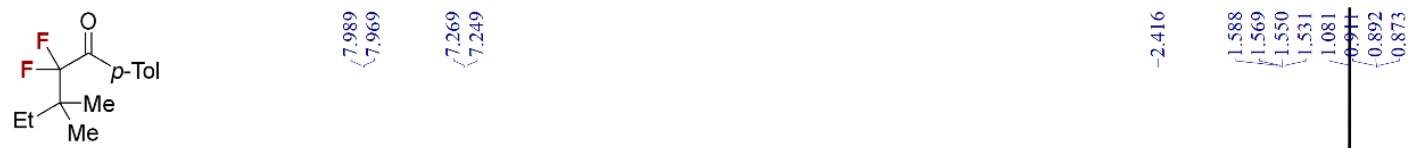

\begin{tabular}{ll}
\multicolumn{1}{c}{ Parameters } \\
Parameter & \multicolumn{1}{c}{ Value } \\
Solvent & CDCl3 \\
Number of Scans & 8 \\
Spectrometer Frequency & 399.69 \\
Nucleus & $1 \mathrm{H}$
\end{tabular}

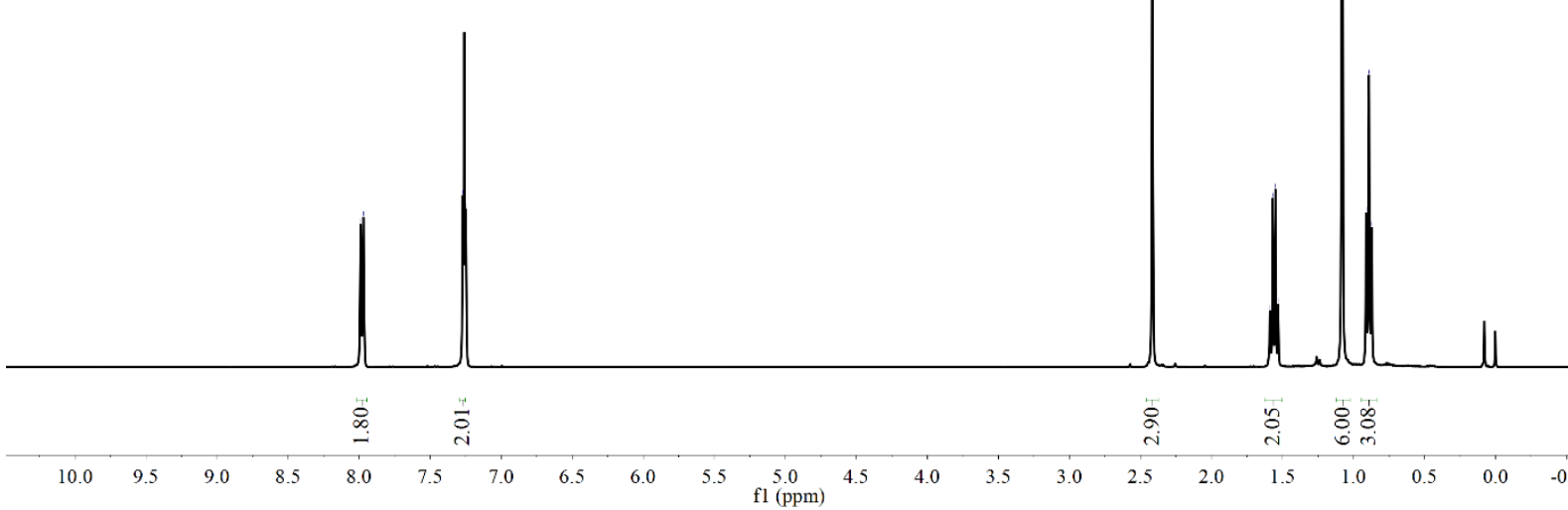


$\overbrace{\mathrm{Me}}^{\mathrm{Me}}$

\begin{tabular}{ll}
\multicolumn{2}{c}{ Parameters } \\
Parameter & \multicolumn{1}{c}{ Value } \\
Solvent & $\mathrm{CDCl} 3$ \\
Number of Scans & 16 \\
Spectrometer Frequency 376.05 \\
Nucleus & $19 \mathrm{~F}$
\end{tabular}

\begin{tabular}{|c|c|}
\hline \multicolumn{2}{|c|}{ Parameters } \\
\hline Parameter & Value \\
\hline Solvent & $\mathrm{CDCl} 3$ \\
\hline Number of Scans & 82 \\
\hline Spectrometer Fre & y 100.62 \\
\hline Nucleus & $13 \mathrm{C}$ \\
\hline
\end{tabular}


2,2-Difluoro-2-(1-methylcyclohexyl)-1-(p-tolyl)ethan-1-one (3i)

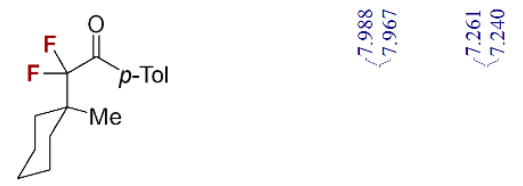

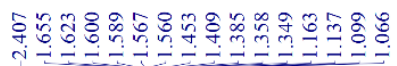

Parameters

Parameter Value

Solvent $\quad \mathrm{CDCl} 3$

Number of Scans 8

Spectrometer Frequency 399.69

Nucleus
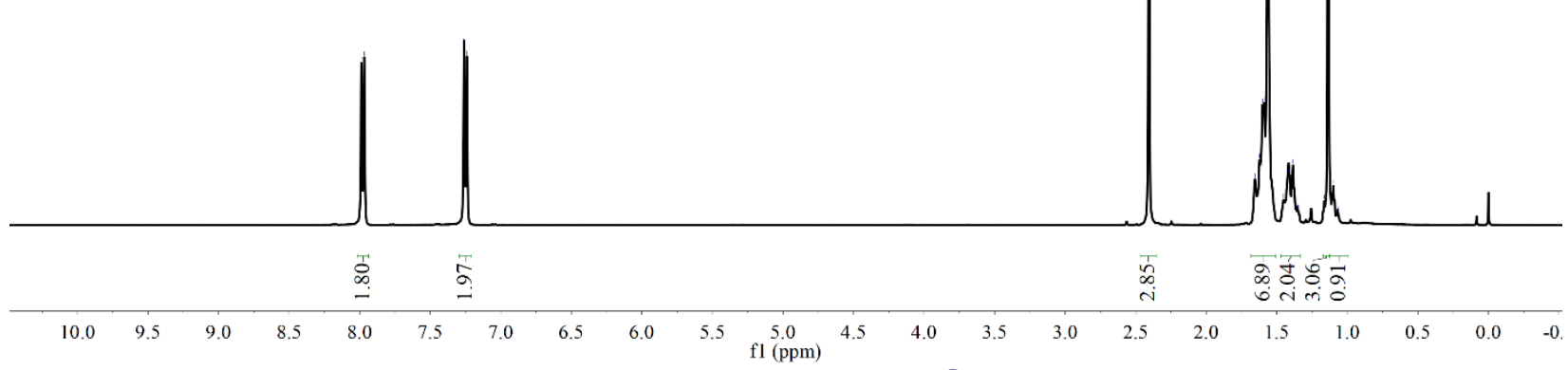

$\overbrace{\mathrm{Me}}^{\mathrm{Mel}}$

음

\begin{tabular}{ll}
\multicolumn{2}{c}{ Parameters } \\
Parameter & \multicolumn{1}{c}{ Value } \\
Solvent & CDCl3 \\
Number of Scans & 16 \\
Spectrometer Frequency 376.05 \\
Nucleus & $19 \mathrm{~F}$
\end{tabular}

$\begin{array}{lllllllllllllllllllllllll}30 & 20 & 10 & 0 & -10 & -20 & -30 & -40 & -50 & -60 & -70 & -80 & -90 & -100 & -110 & -120 & -130 & -140 & -150 & -160 & -170 & -180 & -190 & -200\end{array}$ 


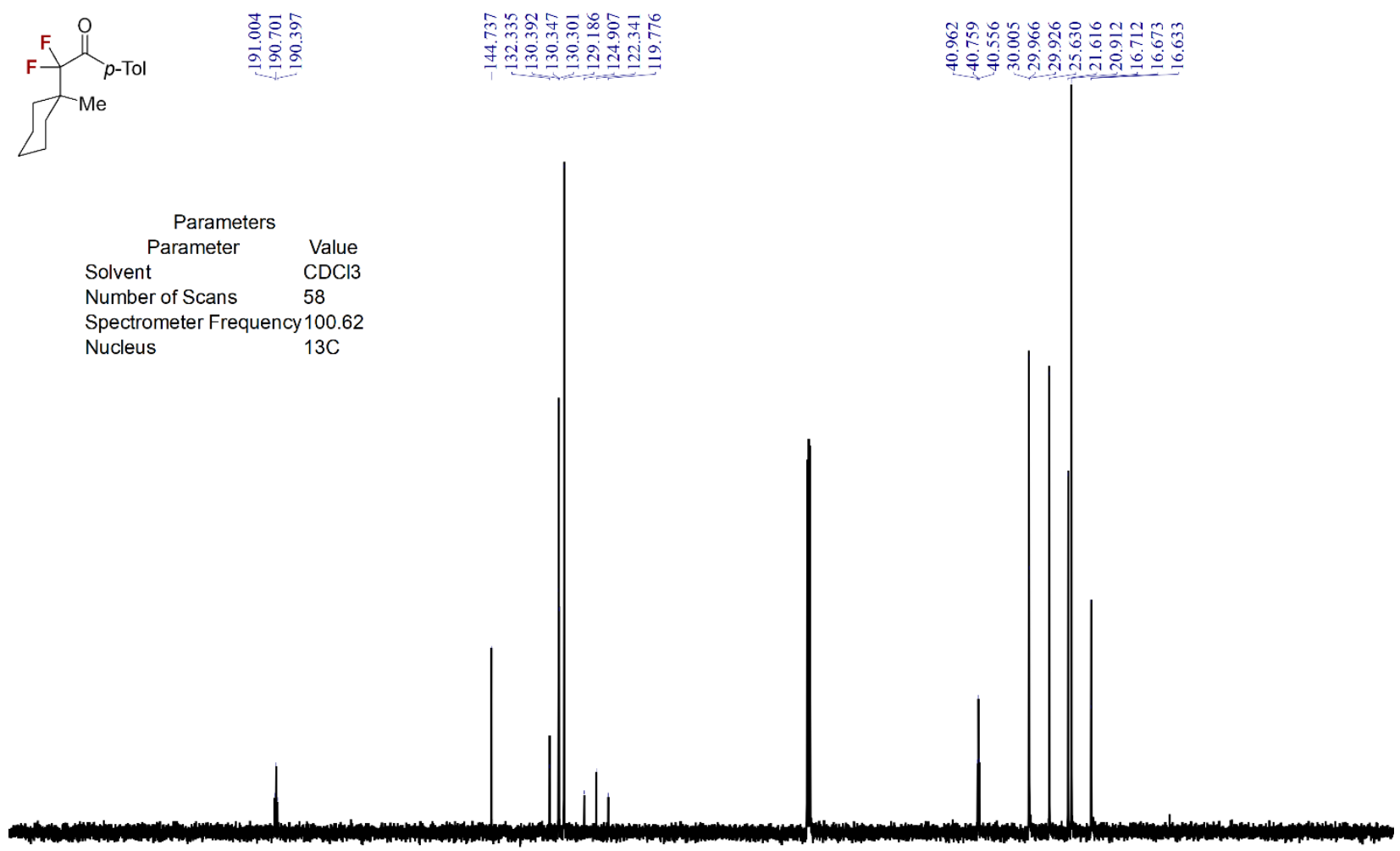

\begin{tabular}{llllllllllllllllllllllllllllllllllll}
\hline 245 & 235 & 225 & 215 & 205 & 195 & 185 & 175 & 165 & 155 & 145 & 135 & 125 & 115 & $\begin{array}{l}105 \\
\mathrm{fl}(\mathrm{ppm})\end{array}$ & 85 & 75 & 65 & 55 & 45 & 35 & 25 & 15 & 5 & -5 & -15 & -25 & -35 & -45
\end{tabular}

\section{2-(Adamantan-1-yl)-2,2-difluoro-1-(p-tolyl)ethan-1-one (3j)}
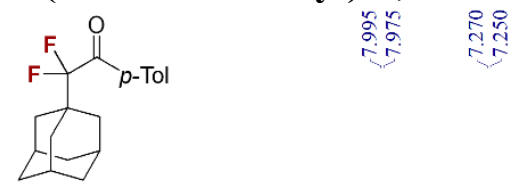

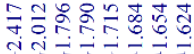

Parameters

Parameter Value

Solvent

Number of Scans

$\mathrm{CDCl} 3$

Spectrometer Frequency 399.69

Nucleus

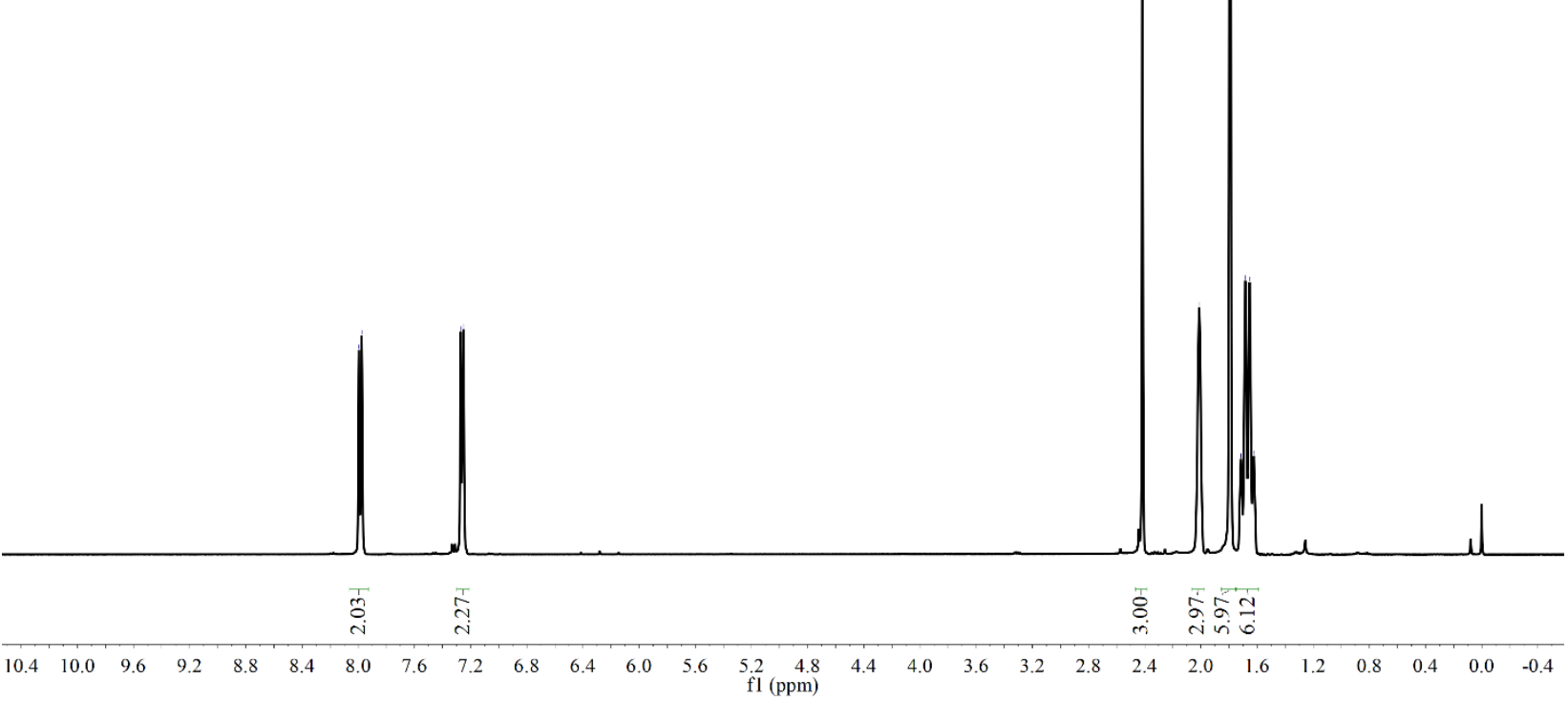




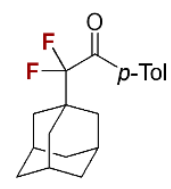

\begin{tabular}{ll}
\multicolumn{1}{c}{ Parameters } \\
Parameter & Value \\
Solvent & $\mathrm{CDCl} 3$ \\
Number of Scans & 16 \\
Spectrometer Frequency & 376.05 \\
Nucleus & $19 \mathrm{~F}$
\end{tabular}

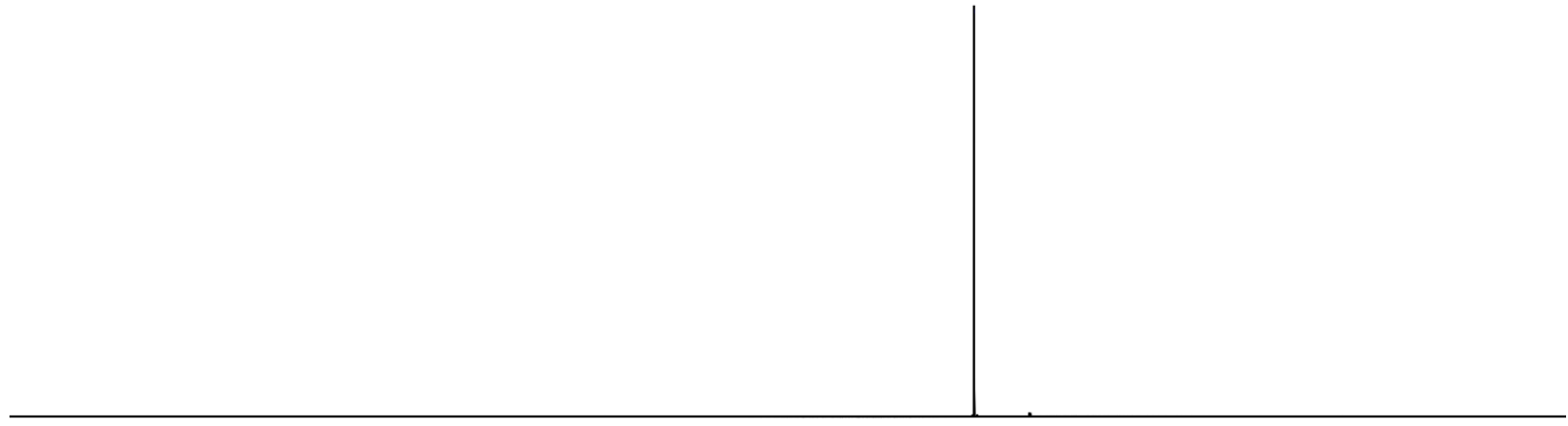

\begin{tabular}{llllllllllllllllllllllll}
\hline 30 & 20 & 10 & 0 & -10 & -20 & -30 & -40 & -50 & -60 & -70 & -80 & -90 & -100 & -110 & -120 & -130 & -140 & -150 & -160 & -170 & -180 & -190 & -200
\end{tabular}

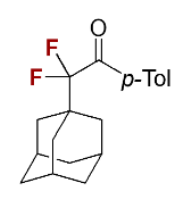

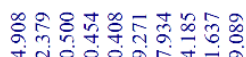

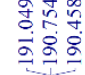

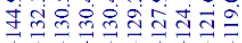

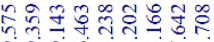

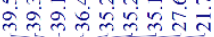

Parameters

Parameter Value

Solvent $\mathrm{CDCl} 3$

Number of Scans $\quad 126$

Spectrometer Frequency 100.62

Nucleus

$13 \mathrm{C}$

$\begin{array}{lllllllllllllll}245 & 235 & 225 & 215 & 205 & 195 & 185 & 175 & 165 & 155 & 145 & 135 & 125 & 115 & \begin{array}{c}105 \\ \mathrm{fl}(\mathrm{ppm})\end{array}\end{array}$ 


\section{2,2-Difluoro-1-(p-tolyl)butan-1-one (3k)}
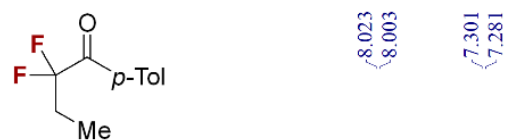

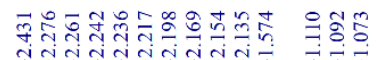

$\mathrm{Me}$

\begin{tabular}{ll}
\multicolumn{2}{c}{ Parameters } \\
Parameter & Value \\
Solvent & $\mathrm{CDCl} 3$ \\
Number of Scans & 8 \\
Spectrometer Frequency 399.69 \\
Nucleus & $1 \mathrm{H}$
\end{tabular}

Nucleus
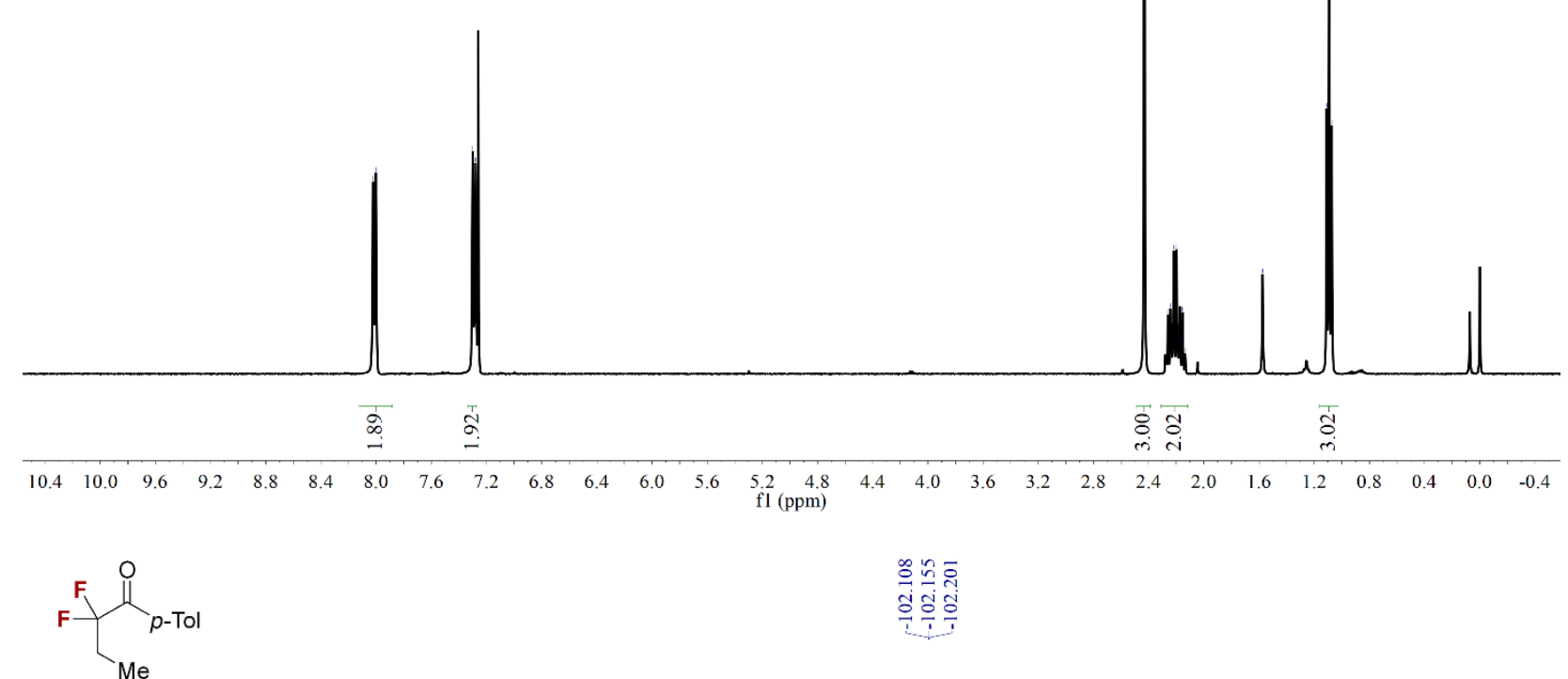

응 옥

성충

$\mathrm{Me}$

\begin{tabular}{ll}
\multicolumn{1}{c}{ Parameters } \\
Parameter & \multicolumn{1}{c}{ Value } \\
Solvent & $\mathrm{CDCl} 3$ \\
Number of Scans & 16 \\
Spectrometer Frequency 376.05 \\
Nucleus & $19 \mathrm{~F}$
\end{tabular}

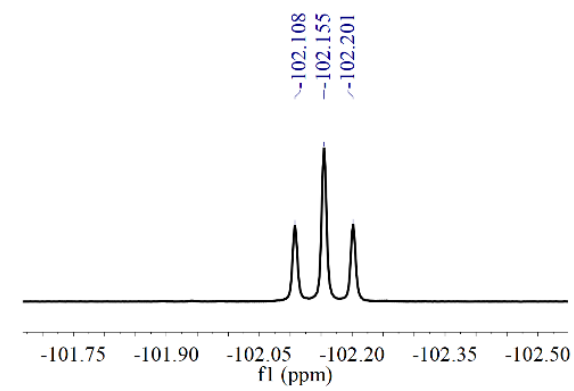

$\begin{array}{llllllllllllllllllllllllll}30 & 20 & 10 & 0 & -10 & -20 & -30 & -40 & -50 & -60 & -70 & -80 & -90 & -100 & -110 & -120 & -130 & -140 & -150 & -160 & -170 & -180 & -190 & -200\end{array}$ 44 


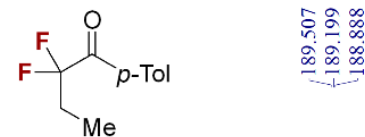

Parameters Parameter Value Solvent

Number of Scans

Spectrometer Frequency 100.62

Nucleus

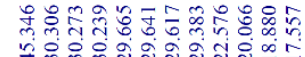

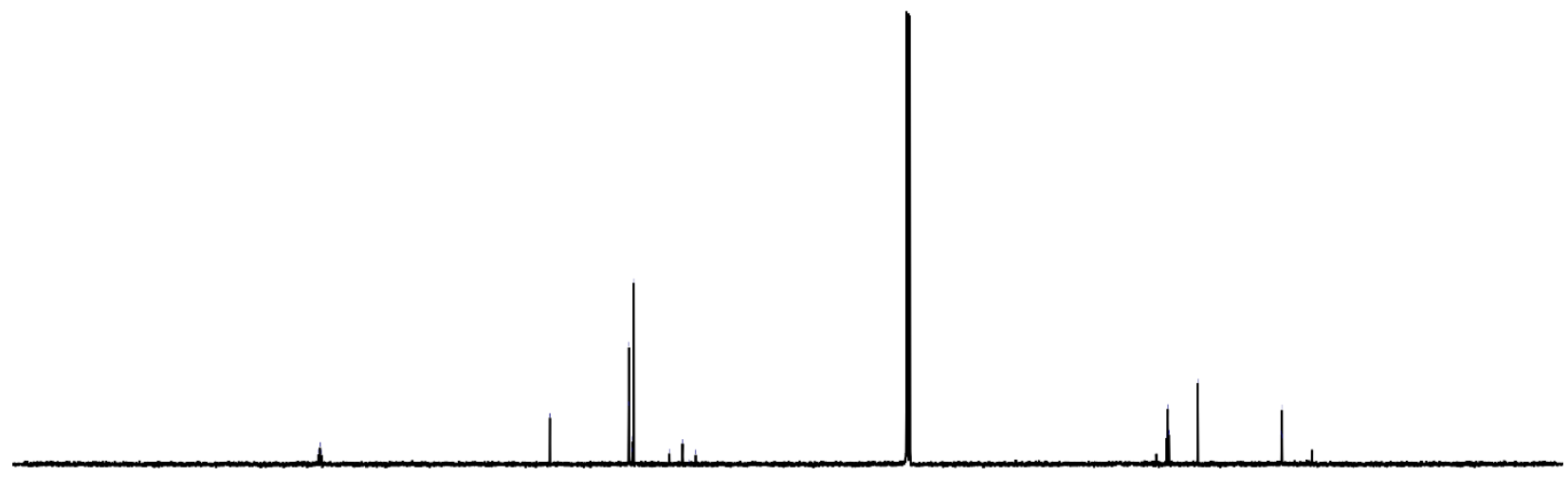

\begin{tabular}{llllllllllllllllllllllllllllllllllllll}
\hline 245 & 235 & 225 & 215 & 205 & 195 & 185 & 175 & 165 & 155 & 145 & 135 & 125 & 115 & $\begin{array}{l}105 \\
\mathrm{fl}(\mathrm{ppm})\end{array}$ & 85 & 75 & 65 & 55 & 45 & 35 & 25 & 15 & 5 & -5 & -15 & -25 & -35 & -45
\end{tabular}

\section{2,2-Difluoro-4-(1H-indol-3-yl)-1-(p-tolyl)butan-1-one (3I)}

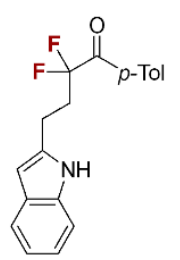

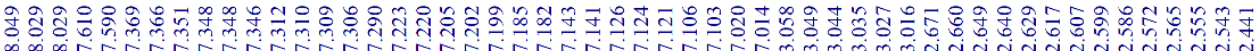

\begin{tabular}{ll}
\multicolumn{1}{c}{ Parameters } \\
Parameter & Value \\
Solvent & $\mathrm{CDCl} 3$ \\
Number of Scans & 8 \\
Spectrometer Frequency 399.69 \\
Nucleus & $1 \mathrm{H}$
\end{tabular}

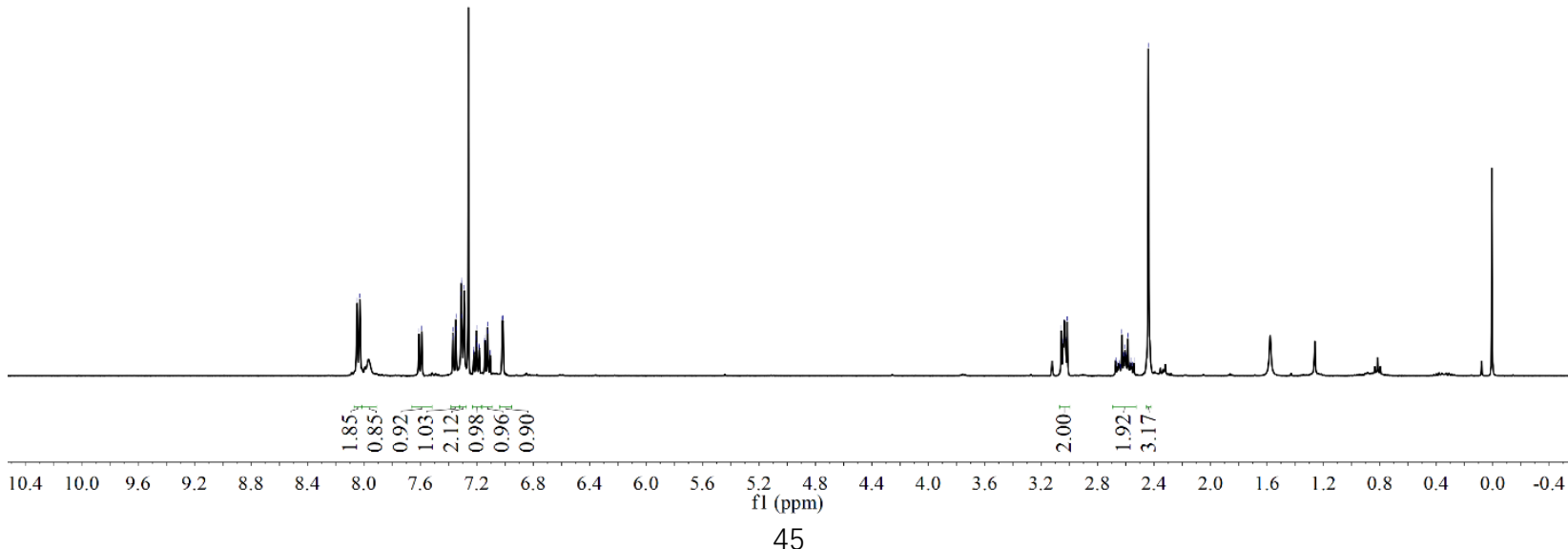




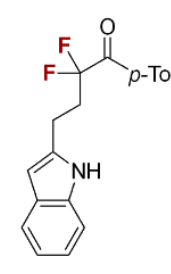

\begin{tabular}{ll}
\multicolumn{1}{c}{ Parameters } \\
Parameter & Value \\
Solvent & $\mathrm{CDCl3}$ \\
Number of Scans & 16 \\
Spectrometer Frequency 376.05 \\
Nucleus & $19 \mathrm{~F}$
\end{tabular}

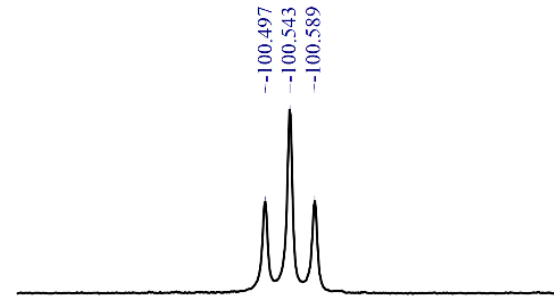

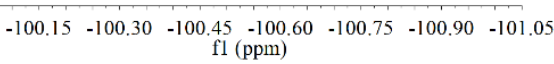

\begin{tabular}{lllllllllllllllllllllllllll}
\hline 30 & 20 & 10 & 0 & -10 & -20 & -30 & -40 & -50 & -60 & -70 & -80 & -90 & -100 & -110 & -120 & -130 & -140 & -150 & -160 & -170 & -180 & -190 & -200
\end{tabular}

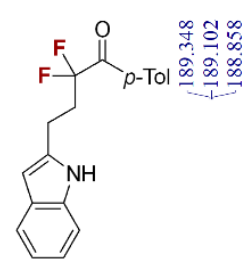

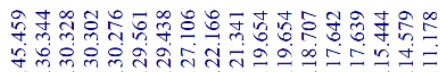

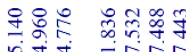

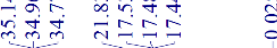

\begin{tabular}{lc}
\multicolumn{1}{c}{ Parameters } \\
Parameter & Value \\
Solvent & CDCl3 \\
Number of Scans & 200 \\
Spectrometer Frequency 125.77 \\
Nucleus & $13 \mathrm{C}$
\end{tabular}

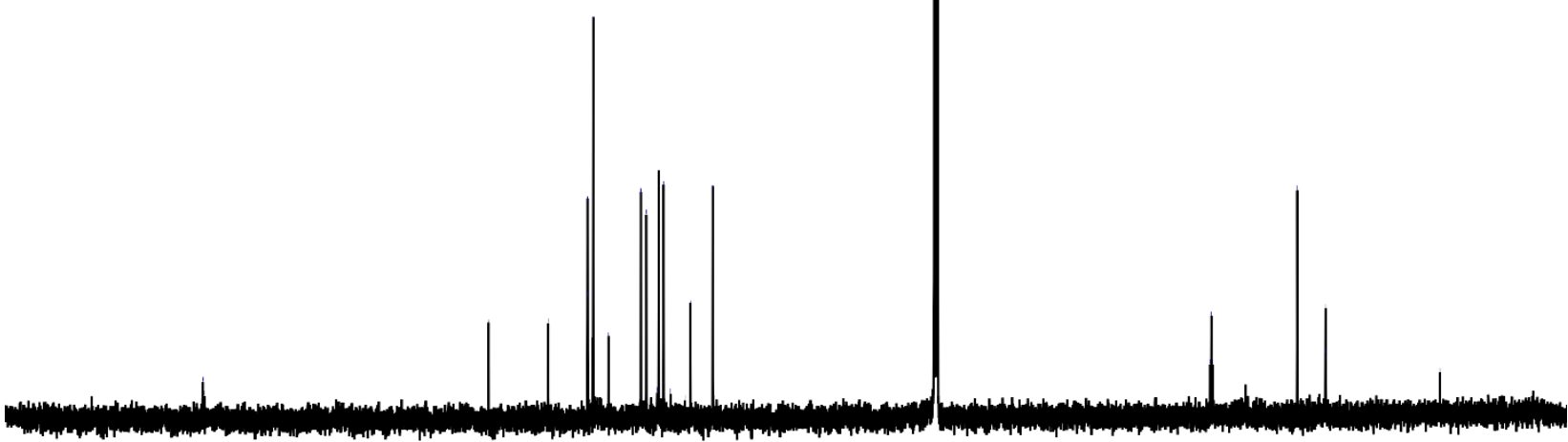

$\begin{array}{lllllllllllll}215 & 205 & 195 & 185 & 175 & 165 & 155 & 145 & 135 & 125 & 115 & \begin{array}{c}105 \\ \text { fl (ppm) }\end{array}\end{array}$ 
tert-Butyl 4-(1,1-difluoro-2-oxo-2-phenylethyl)piperidine-1-carbox-ylate (3m)<smiles>O=CCC1CCNCC1</smiles>
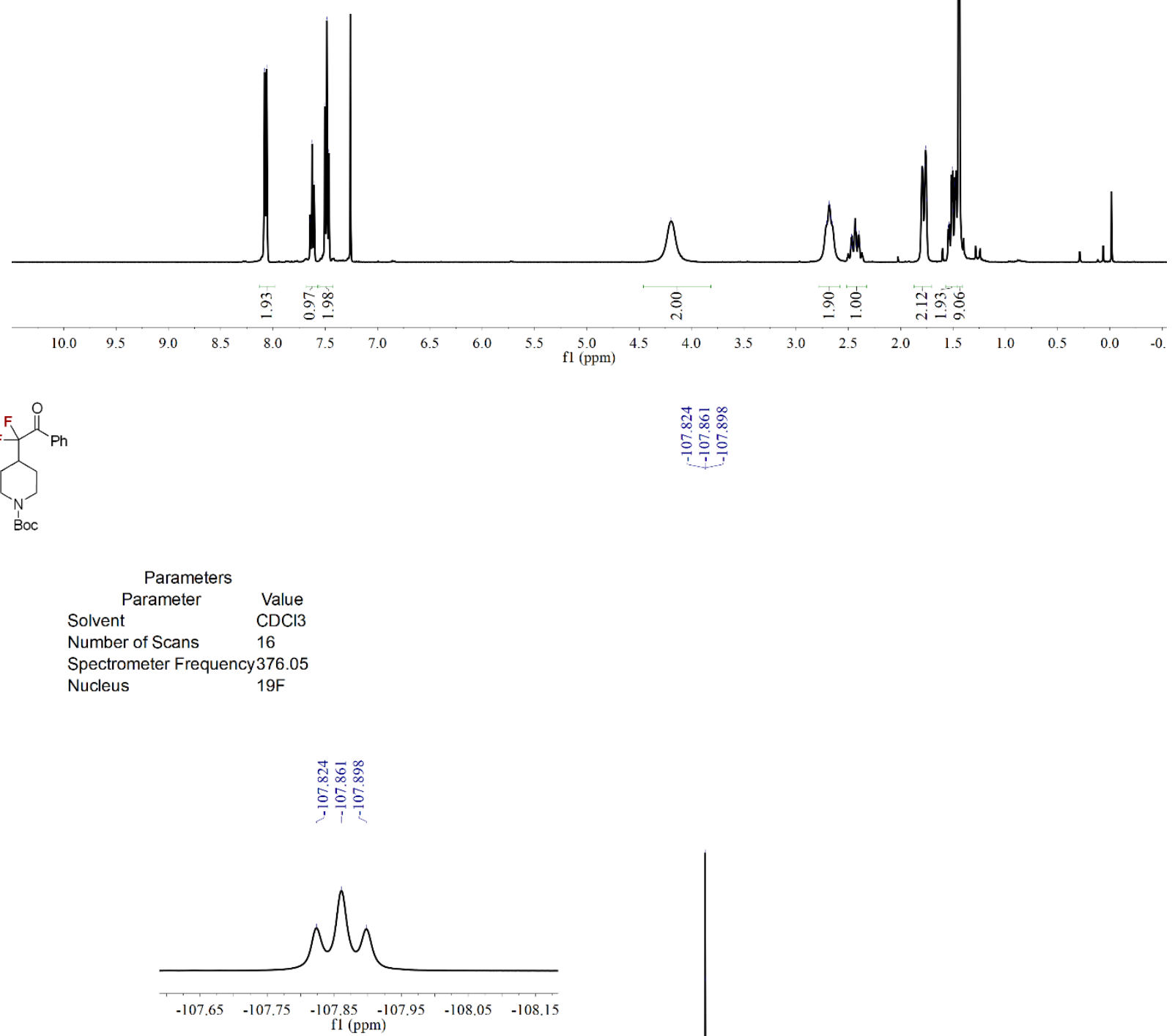

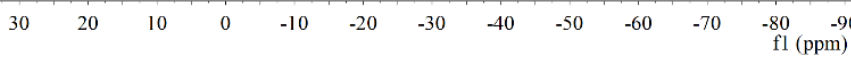




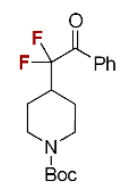

Boc

\begin{tabular}{ll}
\multicolumn{2}{c}{ Parameters } \\
Parameter & Value \\
Solvent & $\mathrm{CDCl} 3$ \\
Number of Scans & 91 \\
Spectrometer Frequency 100.62 \\
Nucleus & $13 \mathrm{C}$
\end{tabular}

Nucleus
Spectromer

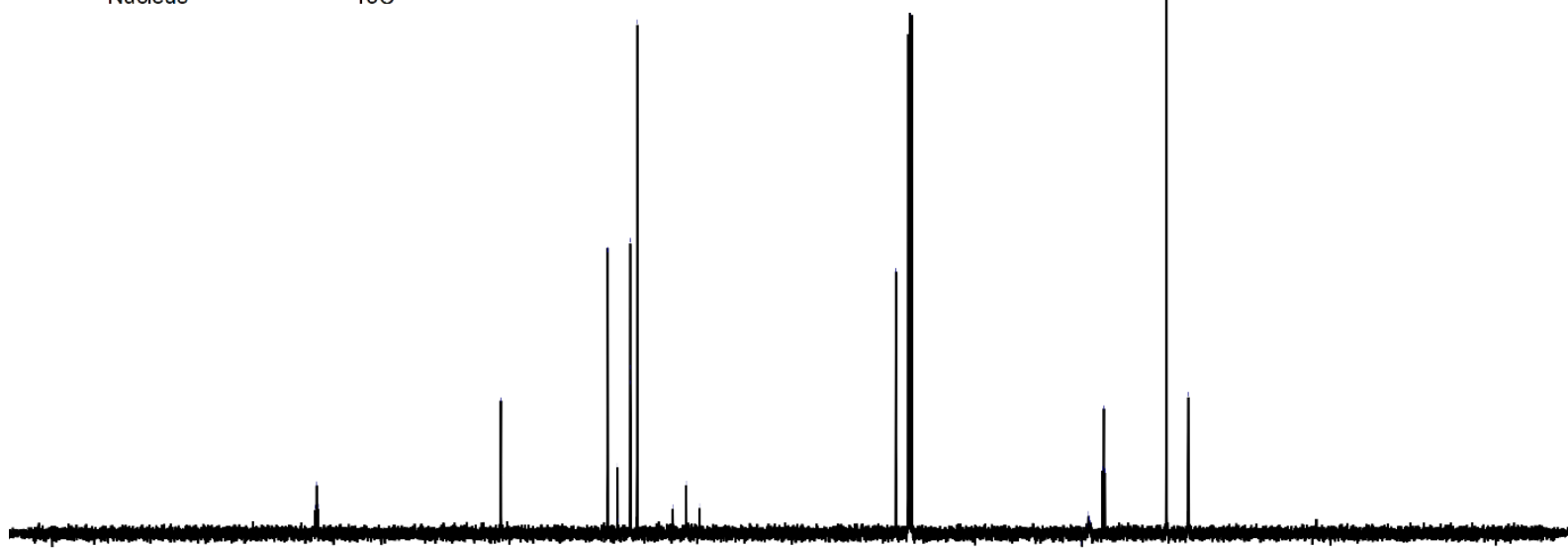

$\begin{array}{llllllllllllllllllllllllllllllll}245 & 235 & 225 & 215 & 205 & 195 & 185 & 175 & 165 & 155 & 145 & 135 & 125 & 115 & \begin{array}{l}105 \\ \text { fl (ppm) }\end{array} & 85 & 75 & 65 & 55 & 45 & 35 & 25 & 15 & 5 & -5 & -15 & -25 & -35 & -45\end{array}$

tert-Butyl 4-(2-(4-(tert-butyl)phenyl)-1,1-difluoro-2-oxoethyl)-piperidine-1-carboxylate (3n)
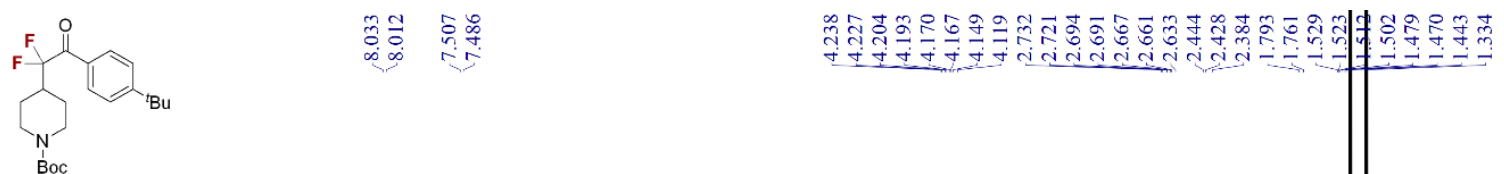

\begin{tabular}{ll}
\multicolumn{2}{c}{ Parameters } \\
Parameter & Value \\
Solvent & $\mathrm{CDCl} 3$ \\
Number of Scans & 8 \\
Spectrometer Frequency 399.69 \\
Nucleus & $1 \mathrm{H}$
\end{tabular}

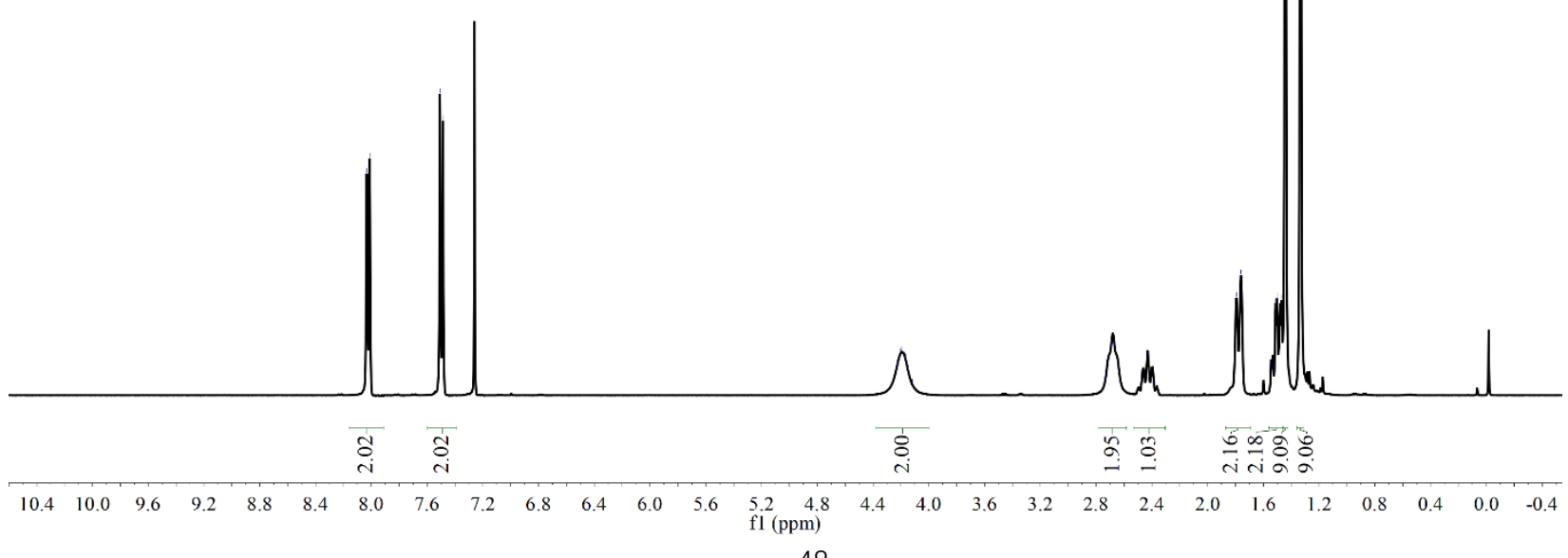




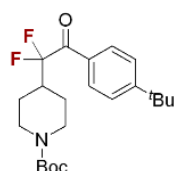

Boc

\begin{tabular}{ll}
\multicolumn{1}{c}{ Parameters } \\
Parameter & Value \\
Solvent & CDCl3 \\
Number of Scans & 16 \\
Spectrometer Frequency 376.05 \\
Nucleus & $19 \mathrm{~F}$
\end{tabular}
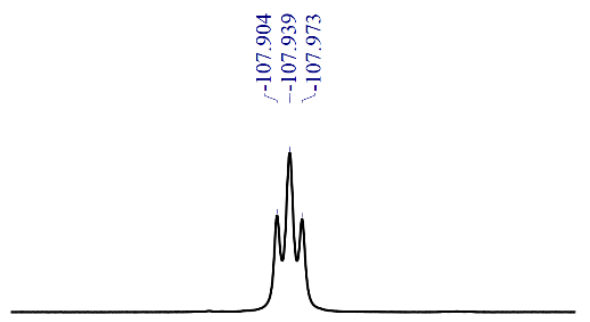

$\begin{array}{lllllllll}-107.3 & -107.5 & -107.7 & -107.9 & -108.1 & -108.3 & -108.5 & -108.7\end{array}$ f1 (ppm)

$30 \quad 20 \quad 10$

$\begin{array}{llll}-10 & -20 & -30\end{array}$

$-40 \quad-50$

$-60-70$

-80
$\mathrm{fl}(\mathrm{ppm})$

$\begin{array}{lllllllllll}-100 & -110 & -120 & -130 & -140 & -150 & -160 & -170 & -180 & -190 & -200\end{array}$

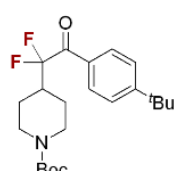

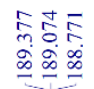

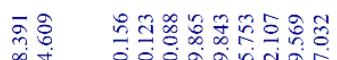

号

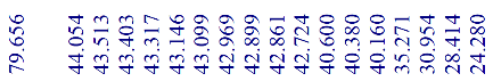

Boc

Parameters

Parameter Value

Solvent $\quad \mathrm{CDCl} 3$

Number of Scans $\quad 146$

Spectrometer Frequency 100.62

Nucleus

$13 \mathrm{C}$

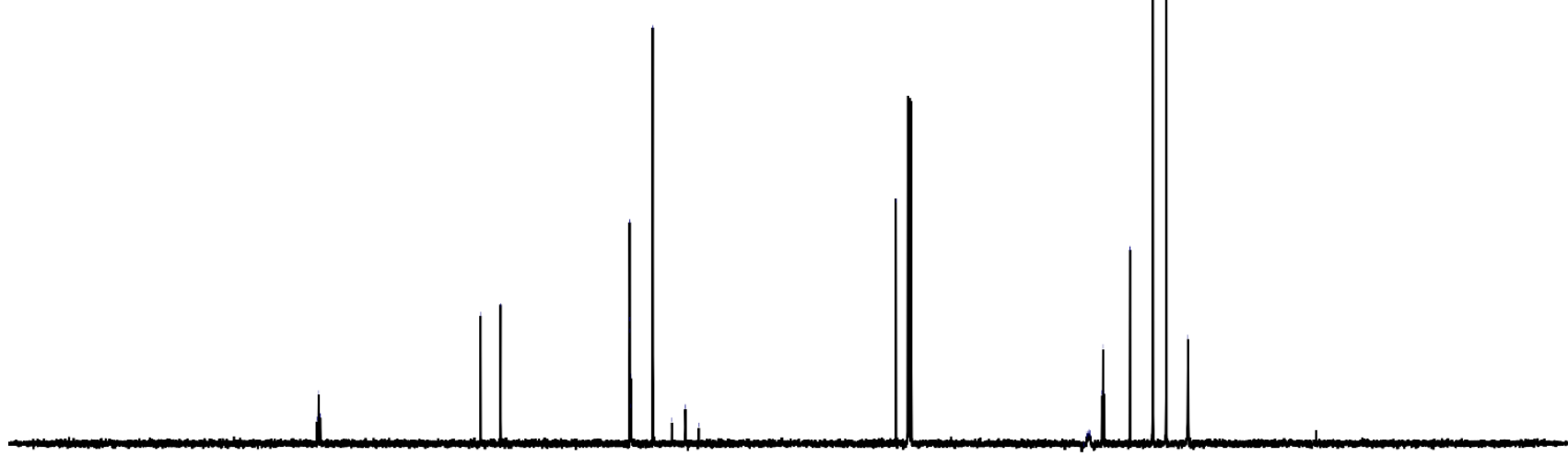

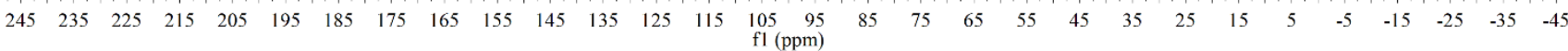




\section{2,2-Difluoro-2-(2-phenylcyclopropyl)-1-(p-tolyl)ethan-1-one (3o)}

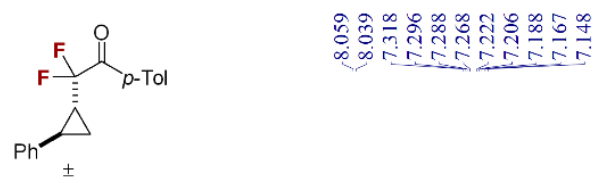

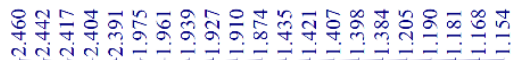

\begin{tabular}{ll}
\multicolumn{2}{c}{ Parameters } \\
Parameter & Value \\
Solvent & $\mathrm{CDCl} 3$ \\
Number of Scans & 8 \\
Spectrometer Frequency 399.69 \\
Nucleus & $1 \mathrm{H}$
\end{tabular}

Nucleus

$1 \mathrm{H}$
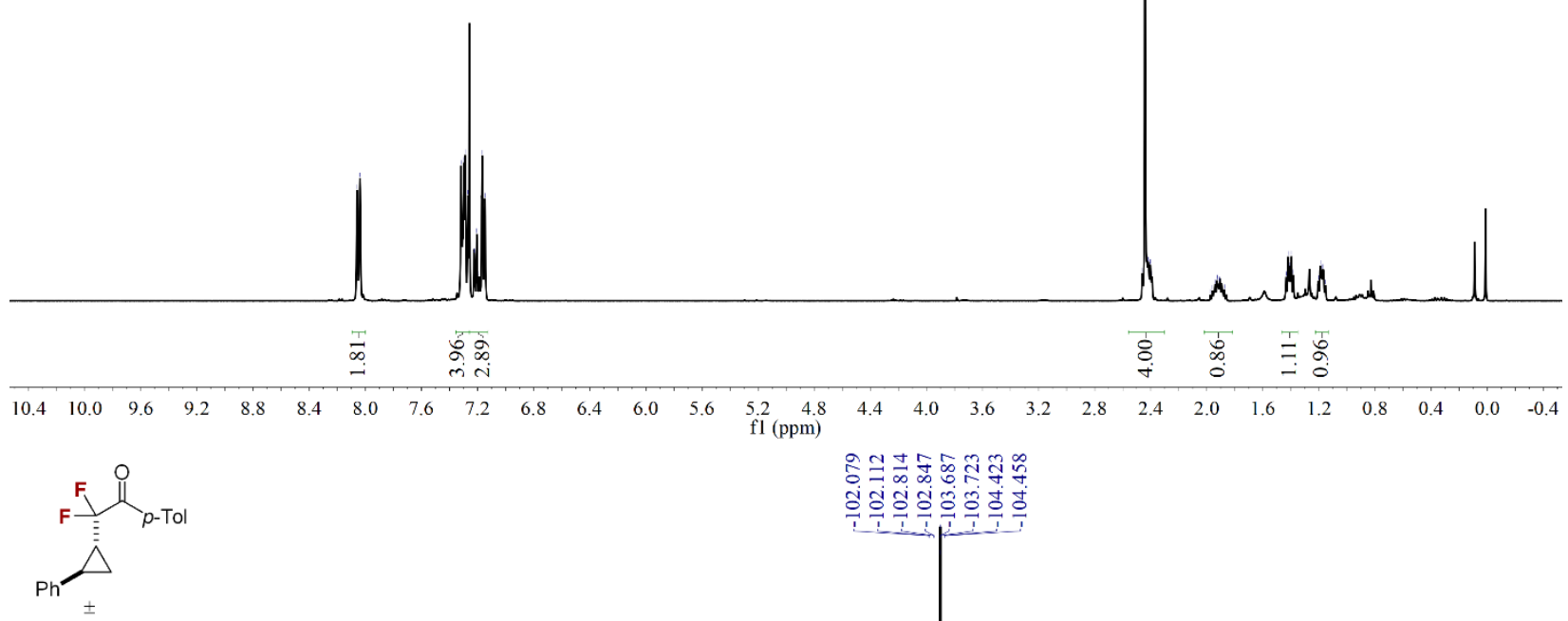

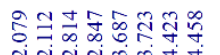

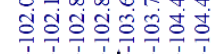

\begin{tabular}{lc}
\multicolumn{2}{c}{ Parameters } \\
Parameter & Value \\
Solvent & $\mathrm{CDCl} 3$ \\
Number of Scans & 16 \\
Spectrometer Frequency 376.05 \\
Nucleus & $19 \mathrm{~F}$
\end{tabular}

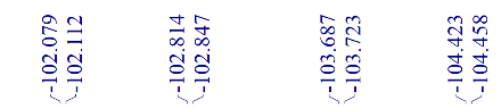

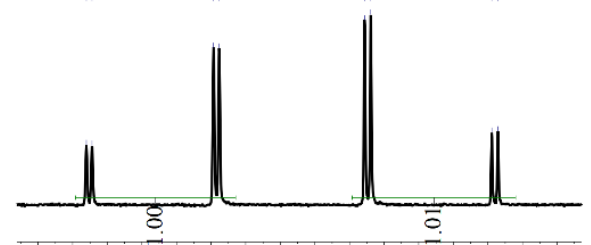

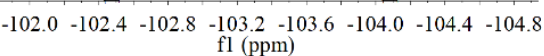

8

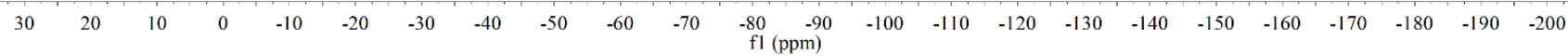




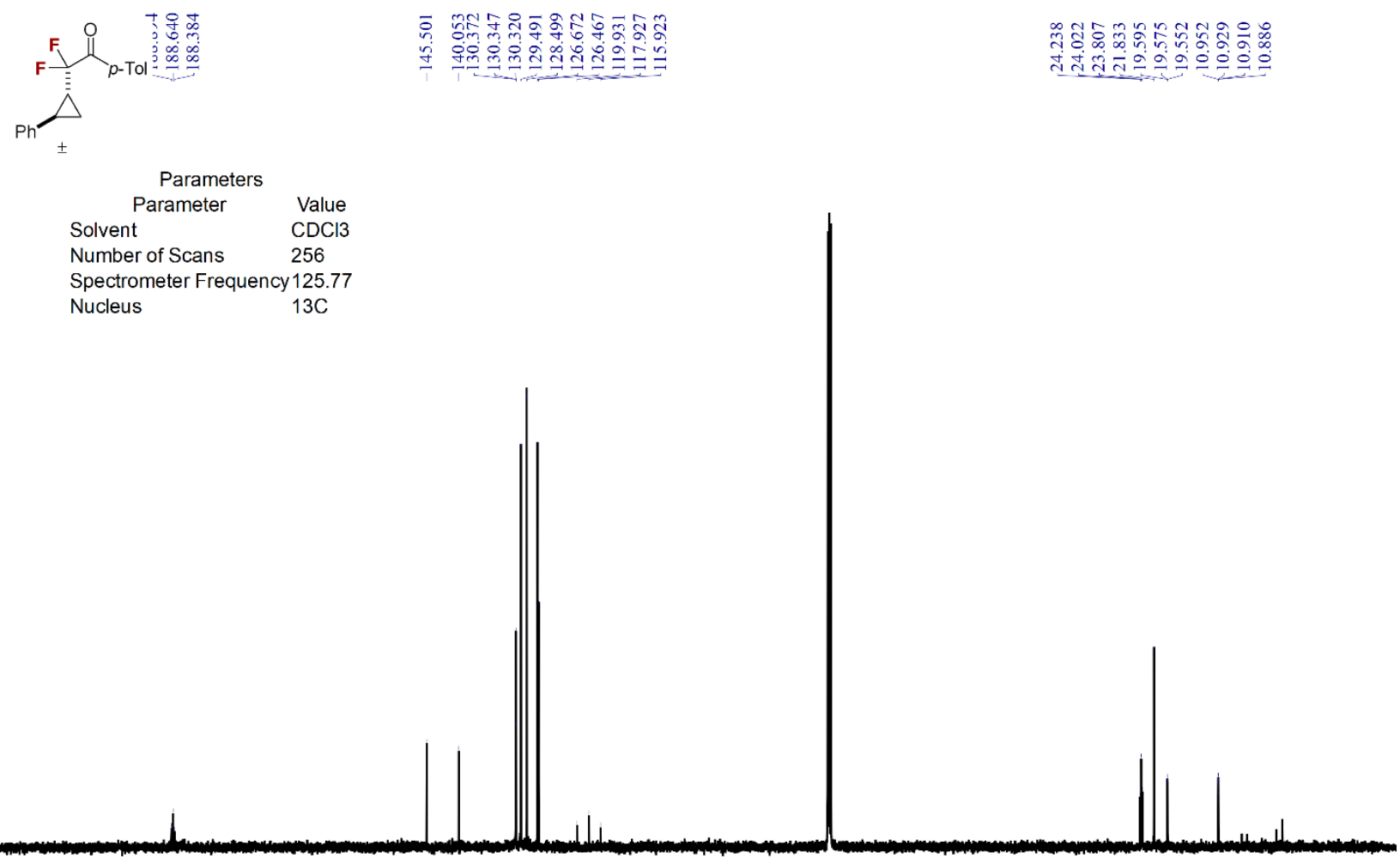

$\begin{array}{lllllllllllllllllllllllll}215 & 205 & 195 & 185 & 175 & 165 & 155 & 145 & 135 & 125 & 115 & \begin{array}{c}105 \\ \mathrm{fl}(\mathrm{ppm})\end{array} & 85 & 75 & 65 & 55 & 45 & 35 & 25 & 15 & 5 & -5 & -15\end{array}$

6-(2,5-Dimethylphenoxy)-2,2-difluoro-3,3-dim-ethyl-1-(p-tolyl)hexan-1-one (3p)

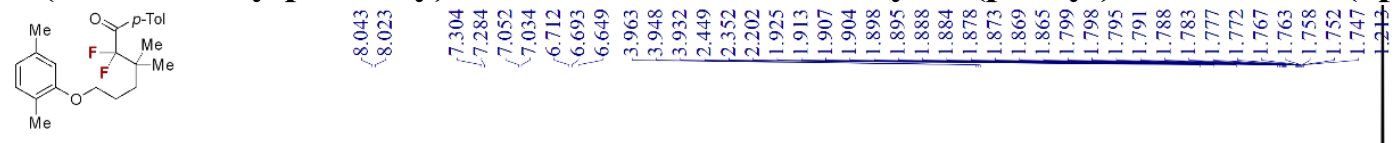

\begin{tabular}{ll}
\multicolumn{1}{c}{ Parameters } \\
Parameter & Value \\
Solvent & $\mathrm{CDCl} 3$ \\
Number of Scans & 8 \\
Spectrometer Frequency & 399.69 \\
Nucleus & $1 \mathrm{H}$
\end{tabular}

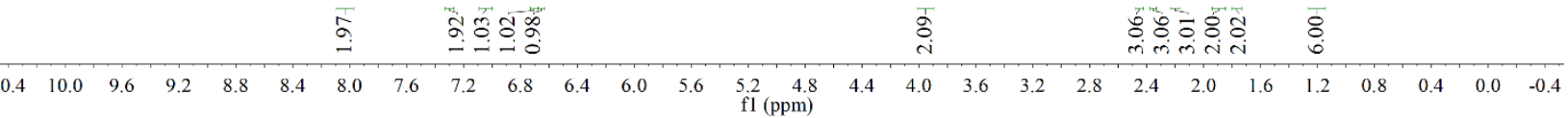




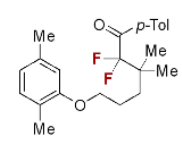

\begin{tabular}{ll}
\multicolumn{2}{c}{ Parameters } \\
Parameter & Value \\
Solvent & CDCl3 \\
Number of Scans & 16 \\
Spectrometer Frequency 376.05 \\
Nucleus & $19 \mathrm{~F}$
\end{tabular}

30

\begin{tabular}{|c|c|c|c|c|c|c|c|c|c|c|c|c|c|c|c|c|}
\hline 20 & 10 & 0 & -10 & -20 & -30 & -40 & -50 & -60 & -70 & $\begin{array}{c}-80 \quad-90 \\
\mathrm{fl}(\mathrm{ppm})\end{array}$ & -100 & -110 & -120 & -130 & -140 & -150 \\
\hline & & & & 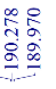 & & & 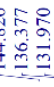 & 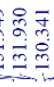 & 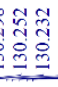 & 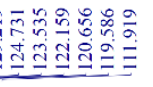 & & $\begin{array}{l}\text { ¿े } \\
\text { o. } \\
\infty \\
\text { i }\end{array}$ & 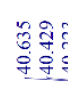 & 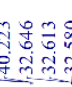 & $\begin{array}{l}0 \\
0 \\
0 \\
0\end{array}$ & 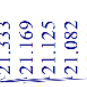 \\
\hline
\end{tabular}

\begin{tabular}{lc}
\multicolumn{2}{c}{ Parameters } \\
Parameter & Value \\
Solvent & $\mathrm{CDCl} 3$ \\
Number of Scans & 508 \\
Spectrometer Frequency & 100.62 \\
Nucleus & $13 \mathrm{C}$
\end{tabular}

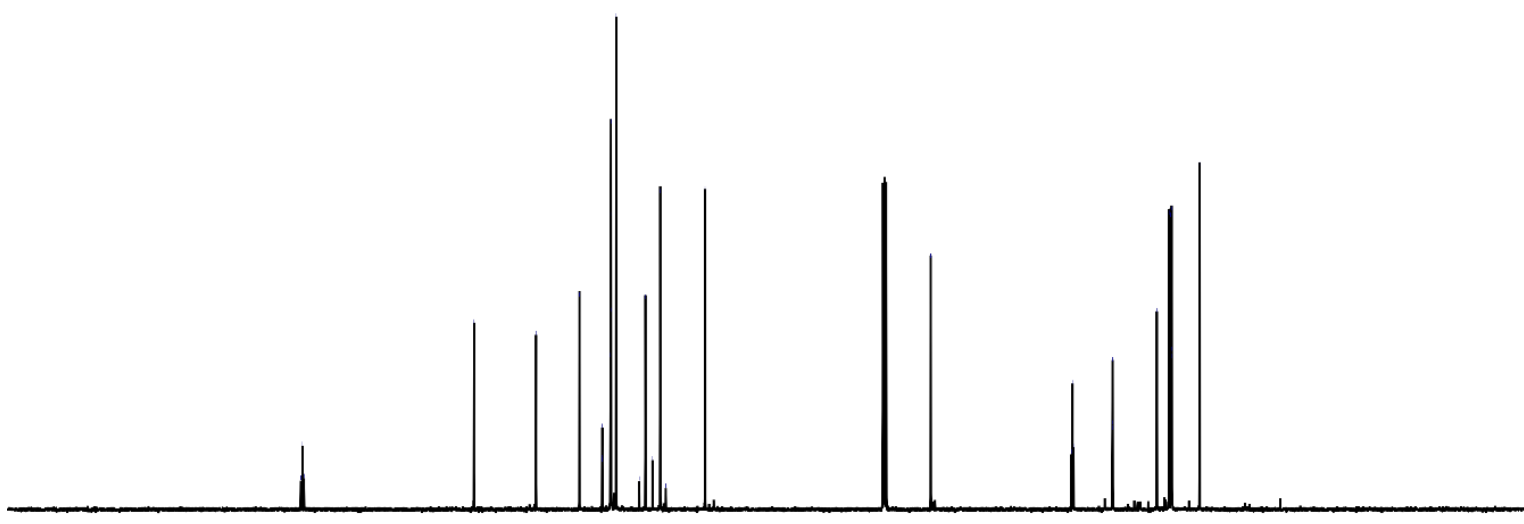

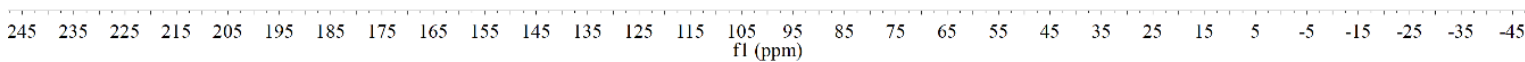


3-(1-(4-Chlorobenzoyl)-5-methoxy-2-methyl-1H-indol-3-yl)-2,2-difluoro-1-(p-tolyl)propan-1one (3q)
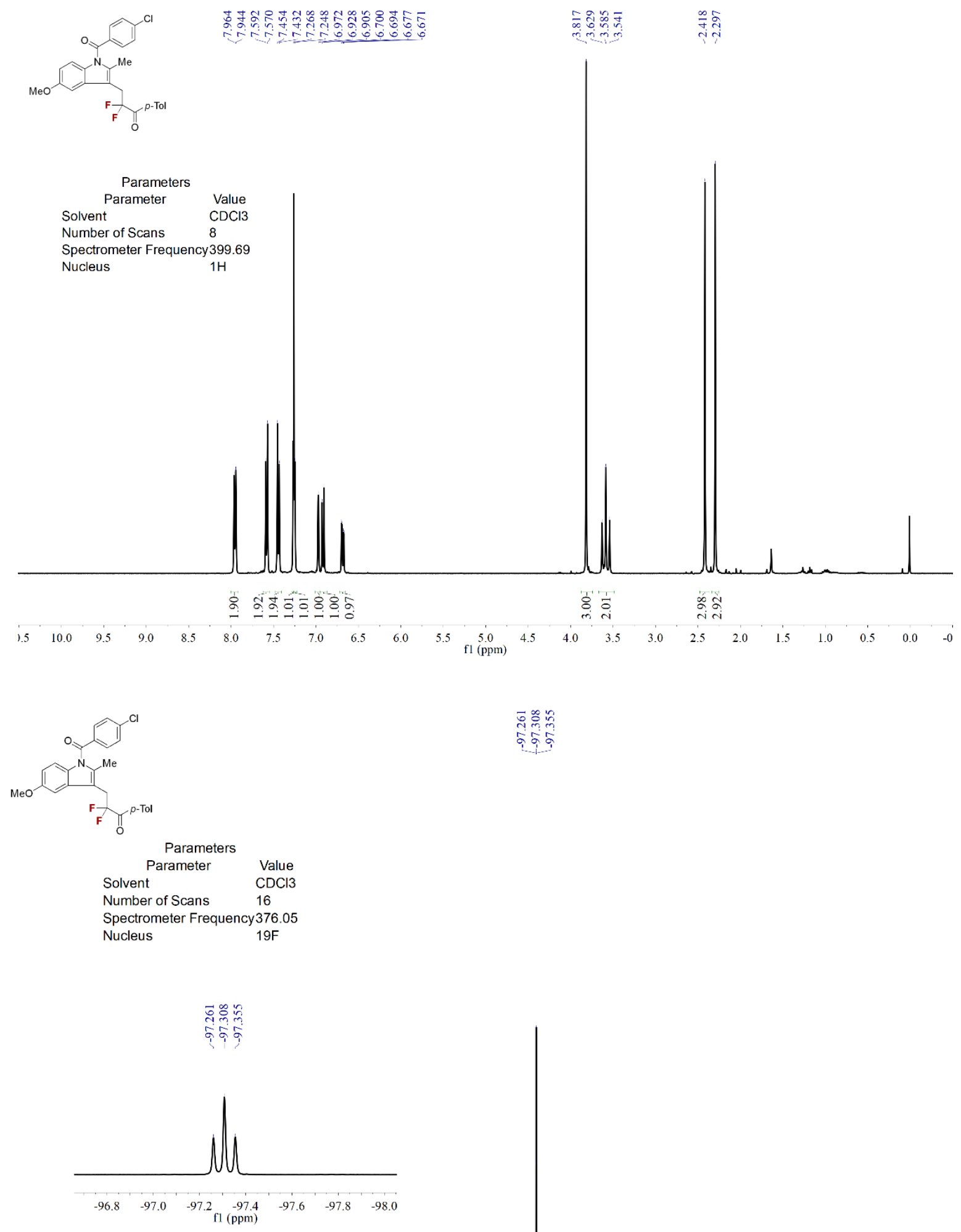

\begin{tabular}{lllllllllllllllllllllllllllllllll}
\hline 30 & 20 & 10 & 0 & -10 & -20 & -30 & -40 & -50 & -60 & -70 & -80 & -90 & -100 & -110 & -120 & -130 & -140 & -150 & -160 & -170 & -180 & -190 & -200
\end{tabular} 


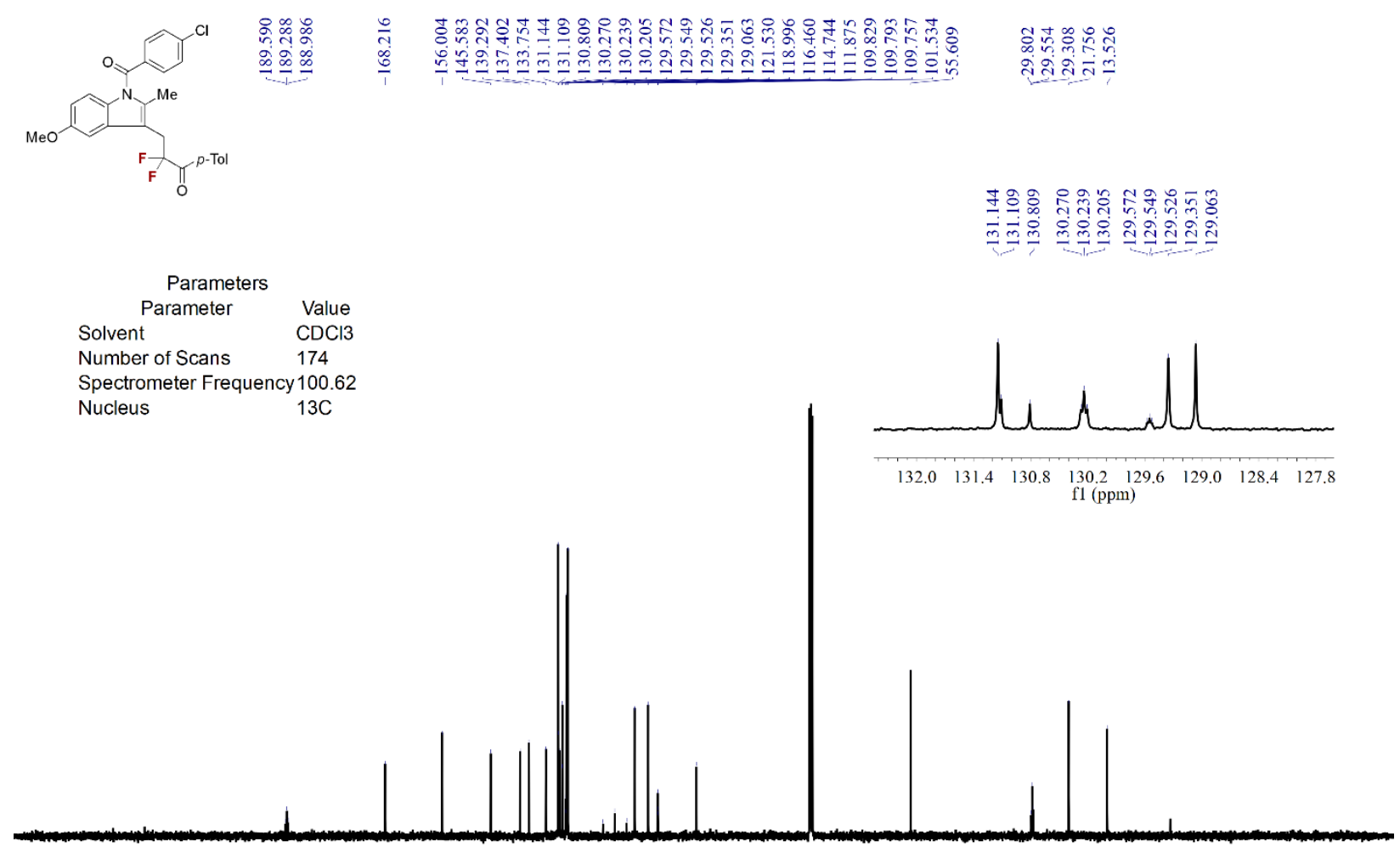

\begin{tabular}{llllllllllllllllllllllllllllllllllll}
\hline 245 & 235 & 225 & 215 & 205 & 195 & 185 & 175 & 165 & 155 & 145 & 135 & 125 & 115 & $\begin{array}{l}105 \\
\mathrm{fl}(\mathrm{ppm})\end{array}$ & 85 & 75 & 65 & 55 & 45 & 35 & 25 & 15 & 5 & -5 & -15 & -25 & -35 & -45
\end{tabular}

Benzyl ((2S)-1-((2,2-difluoro-5-methyl-1-oxo-1-(p-tolyl)hexan-3-yl)amino)-1-oxo-3phenylpropan-2-yl)carbamate (3s)

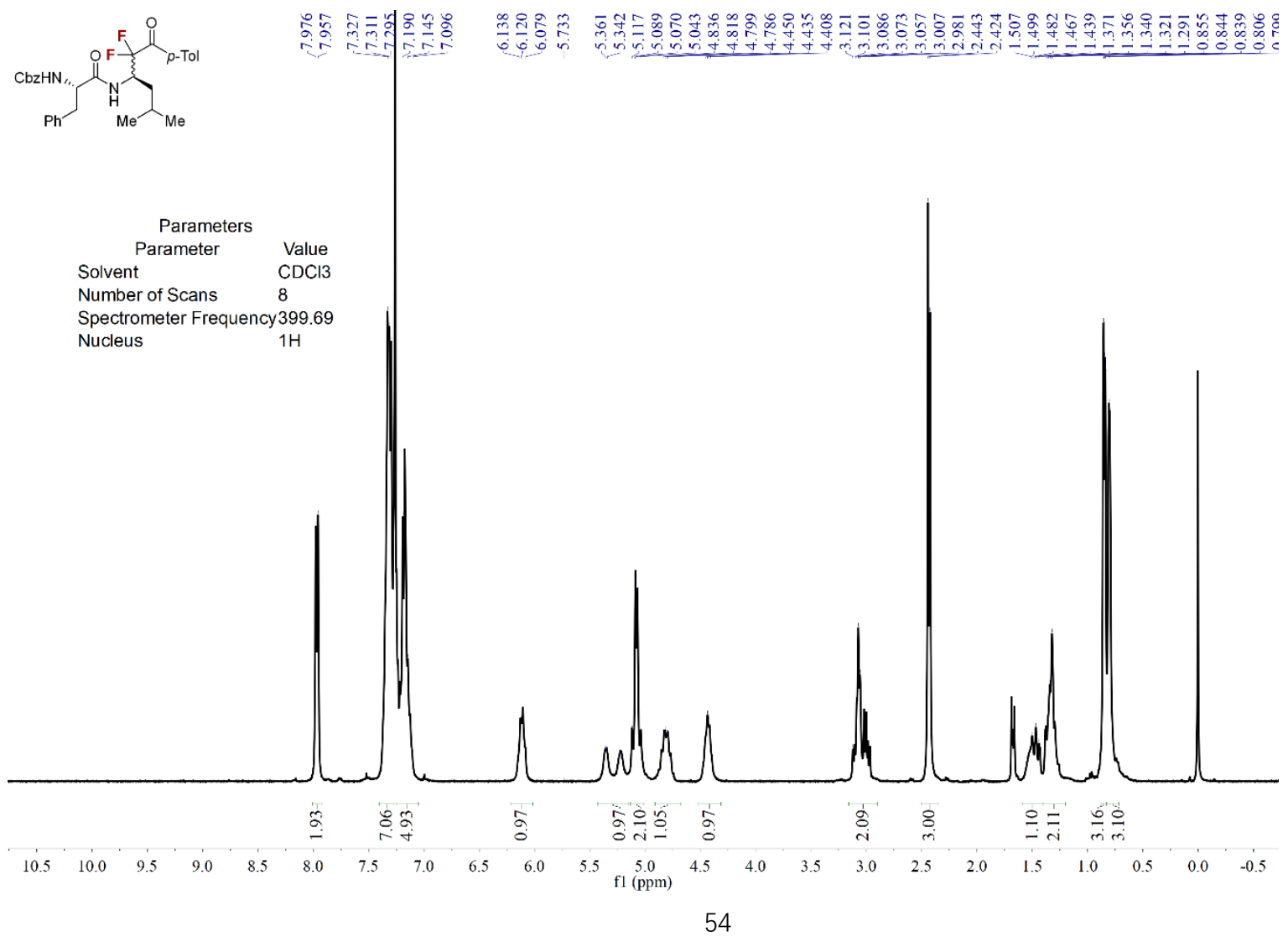




$$
\underbrace{}_{\mathrm{Me}}
$$

$$
\begin{array}{ll}
\multicolumn{1}{c}{\text { Parameters }} \\
\text { Parameter } & \text { Value } \\
\text { Solvent } & \mathrm{CDCl} 3 \\
\text { Number of Scans } & 16 \\
\text { Spectrometer Frequency } & 376.05 \\
\text { Nucleus } & 19 \mathrm{~F}
\end{array}
$$

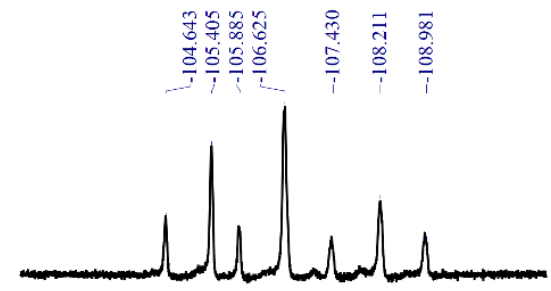

$-102.5-103.5-104.5-105.5-106.5-107.5-108.5-109.5-110.5$ fl (ppm)

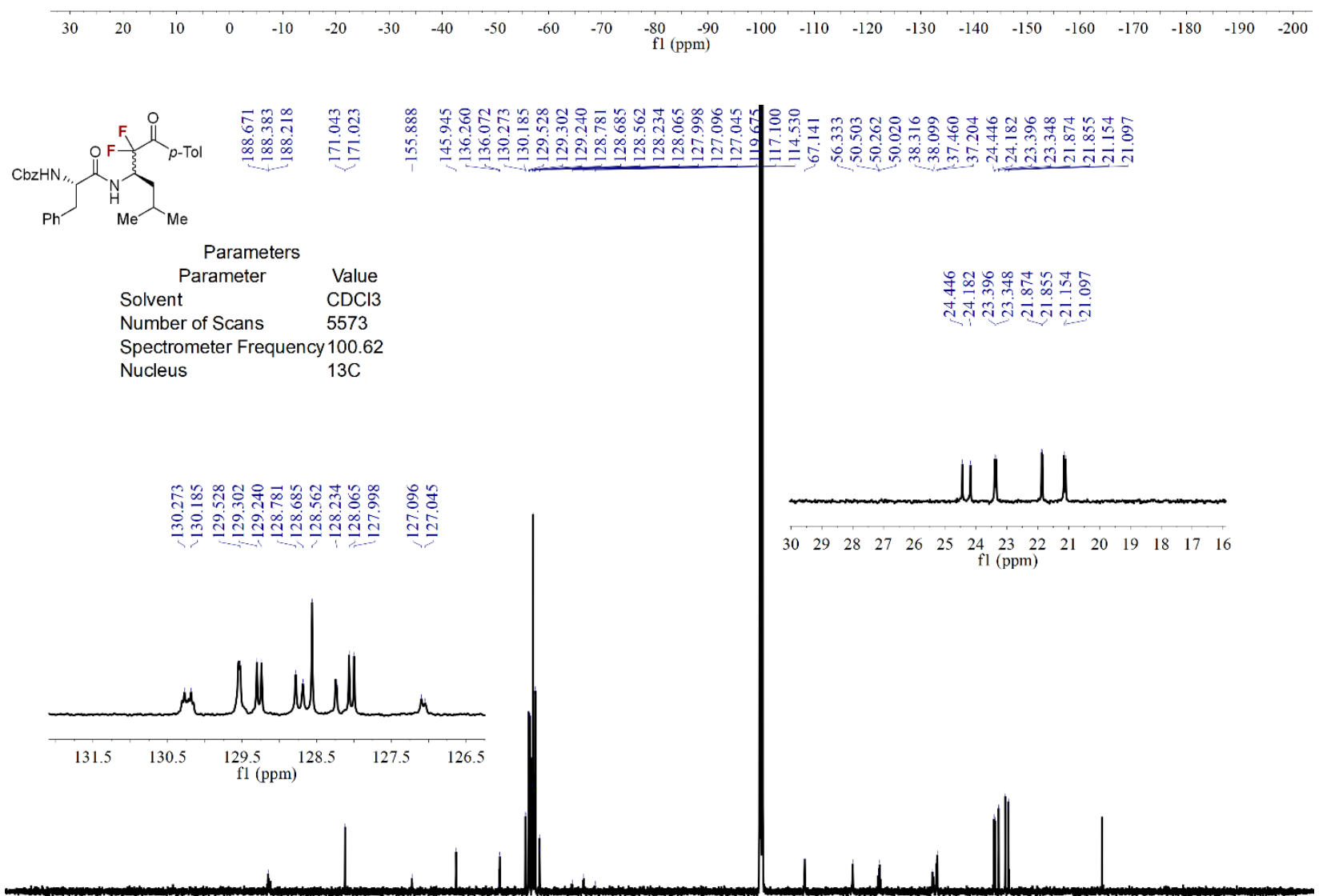

$\begin{array}{lllllllllllllllllllllllllllllllllllllll}245 & 235 & 225 & 215 & 205 & 195 & 185 & 175 & 165 & 155 & 145 & 135 & 125 & 115 & 105 & 95 & 85 & 75 & 65 & 55 & 45 & 35 & 25 & 15 & 5 & -5 & -15 & -25 & -35 & -45\end{array}$ 


\section{Methyl 2-benzyl-3,3-difluoro-4-oxo-4-(p-tolyl)butanoate (3t)}

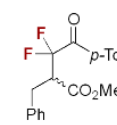

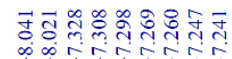

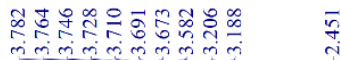

${ }_{\mathrm{Ph}} \mathrm{CO}_{2} \mathrm{Me}$

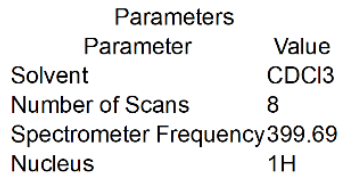

Nucleus $\quad 1 \mathrm{H}$

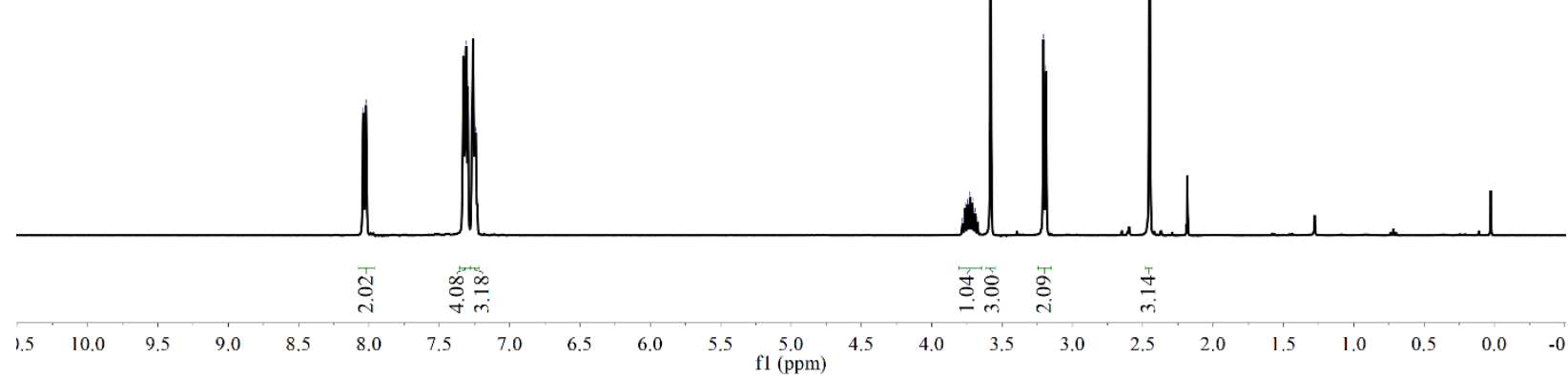

${ }_{\mathrm{Ph}}^{\mathrm{P}} \mathrm{CO}_{2}^{\mathrm{Me}}$

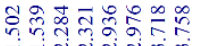

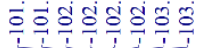

\begin{tabular}{ll}
\multicolumn{2}{c}{ Parameters } \\
Parameter & Value \\
Solvent & CDCl3 \\
Number of Scans & 16 \\
Spectrometer Frequency 376.05 \\
Nucleus & $19 \mathrm{~F}$
\end{tabular}

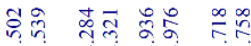

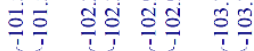

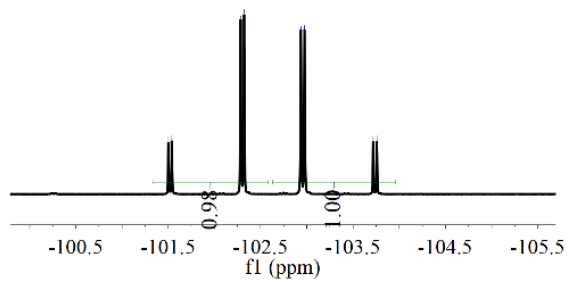

\%̊용

$\begin{array}{lllllllllllllllllllllllllll}30 & 20 & 10 & 0 & -10 & -20 & -30 & -40 & -50 & -60 & -70 & -80 & -90 & -100 & -110 & -120 & -130 & -140 & -150 & -160 & -170 & -180 & -190 & -200\end{array}$ 


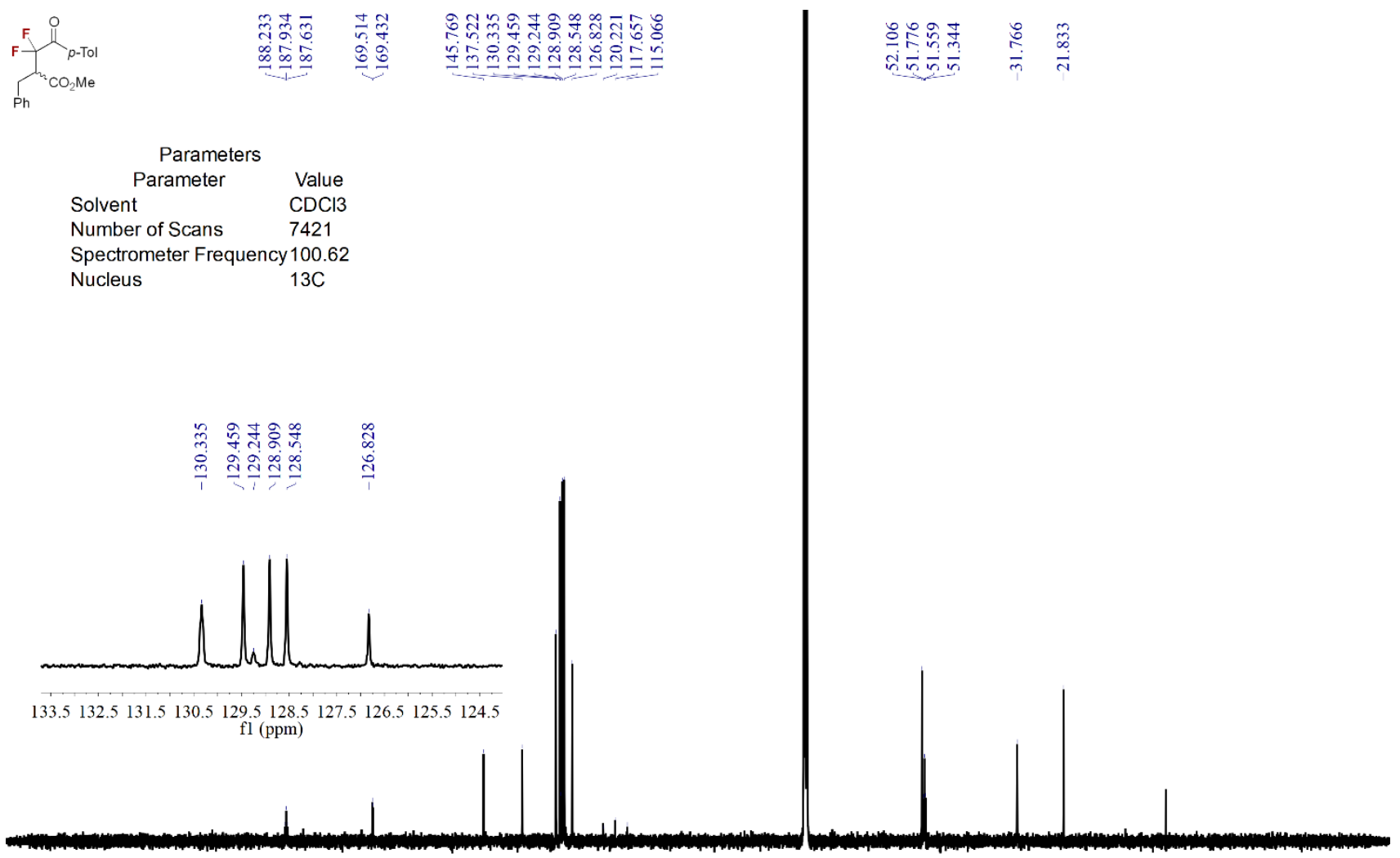

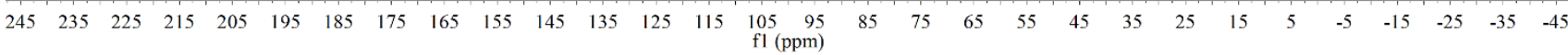

tert-Butyl 4-(difluoromethyl)piperidine-1-carboxylate (5a)

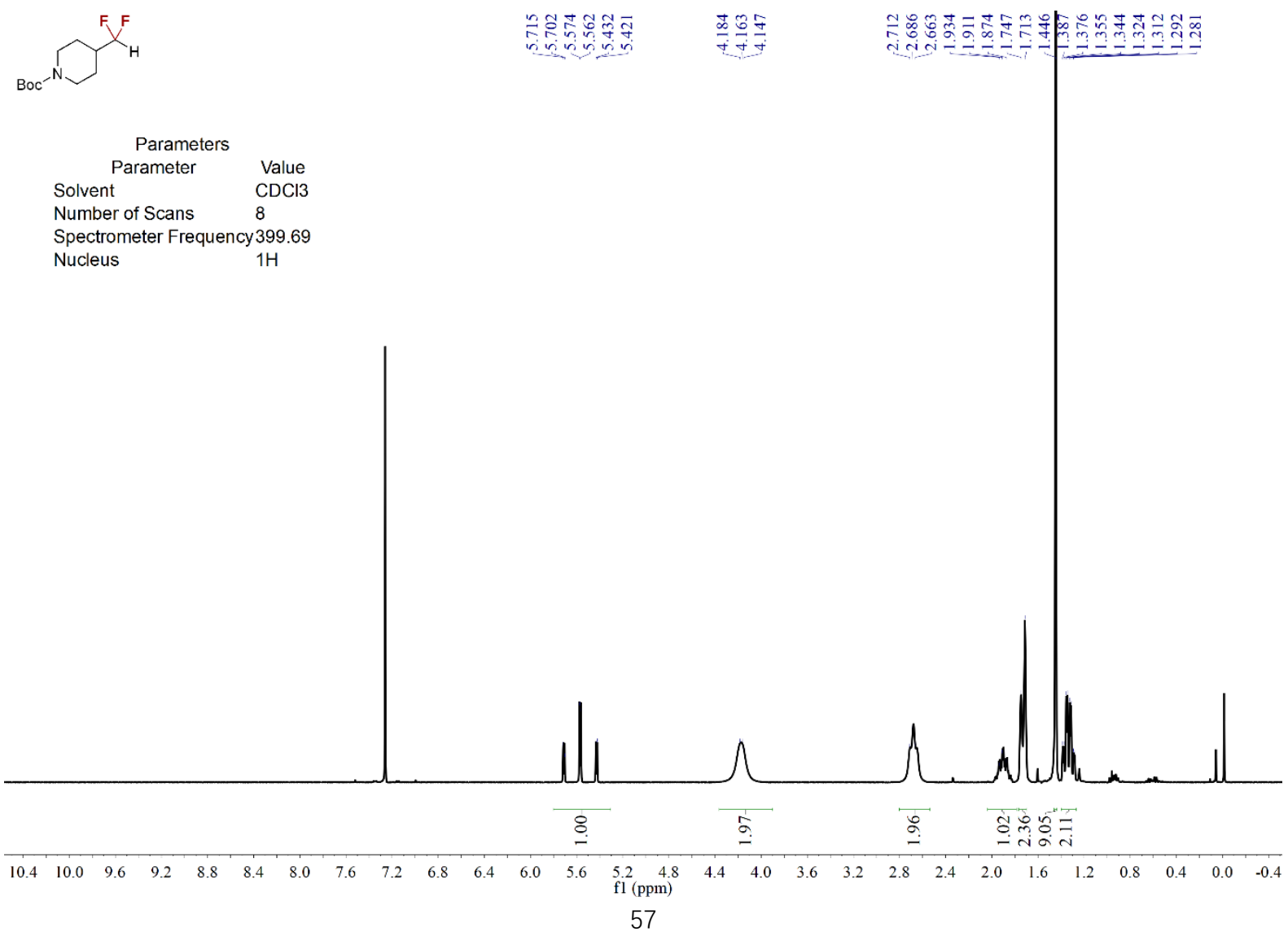


$\overbrace{B c^{-N}}^{F}$

\begin{tabular}{ll}
\multicolumn{1}{c}{$\begin{array}{c}\text { Parameters } \\
\text { Parameter }\end{array}$} & Value \\
Solvent & CDCl3 \\
Number of Scans & 16 \\
Spectrometer Frequency 376.05 \\
Nucleus \\
$19 F$
\end{tabular}

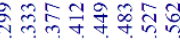

ㄱำงำำำำ

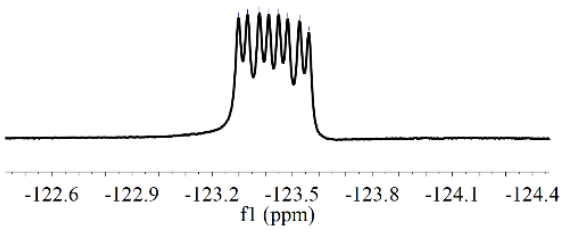

\begin{tabular}{|c|c|c|c|c|c|c|c|c|c|c|c|c|c|c|c|c|c|c|c|c|c|c|}
\hline \multirow[t]{2}{*}{30} & 20 & 10 & 0 & -10 & -20 & -30 & -40 & -50 & -60 & -70 & $\begin{array}{l}-80 \quad-90 \\
\text { fl (ppm) }\end{array}$ & -100 & -110 & -120 & -130 & -140 & -150 & -160 & -170 & -180 & -190 & -200 \\
\hline & & & & & & & $\begin{array}{l}3 \\
0 \\
0 \\
\dot{0} \\
\frac{1}{1}\end{array}$ & & & 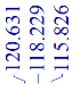 & & $\begin{array}{l}0 \\
0 \\
0 \\
\stackrel{0}{1}\end{array}$ & & 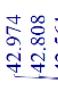 & 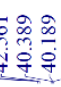 & 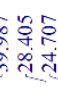 & 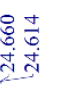 & & & & & \\
\hline
\end{tabular}

\begin{tabular}{ll}
\multicolumn{2}{c}{ Parameters } \\
Parameter & Value \\
Solvent & CDCl3 \\
Number of Scans & 154 \\
Spectrometer Frequency 100.62 \\
Nucleus & $13 \mathrm{C}$
\end{tabular}

\begin{tabular}{lllllllllllllllllllllllllllllllllllll}
\hline 245 & 235 & 225 & 215 & 205 & 195 & 185 & 175 & 165 & 155 & 145 & 135 & 125 & 115 & $\begin{array}{l}105 \\
\mathrm{fl}(\mathrm{ppm})\end{array}$ & 85 & 75 & 65 & 55 & 45 & 35 & 25 & 15 & 5 & -5 & -15 & -25 & -35 & -45
\end{tabular} 
Tert-butyl 3-(difluoromethyl)azetidine-1-carboxylate (5b)<smiles></smiles>

Boc

\begin{tabular}{ll}
\multicolumn{1}{c}{ Parameters } \\
Parameter & \multicolumn{1}{c}{ Value } \\
Solvent & $\mathrm{CDCl} 3$ \\
Number of Scans & 8 \\
Spectrometer Frequency 399.70 \\
Nucleus & $1 \mathrm{H}$
\end{tabular}

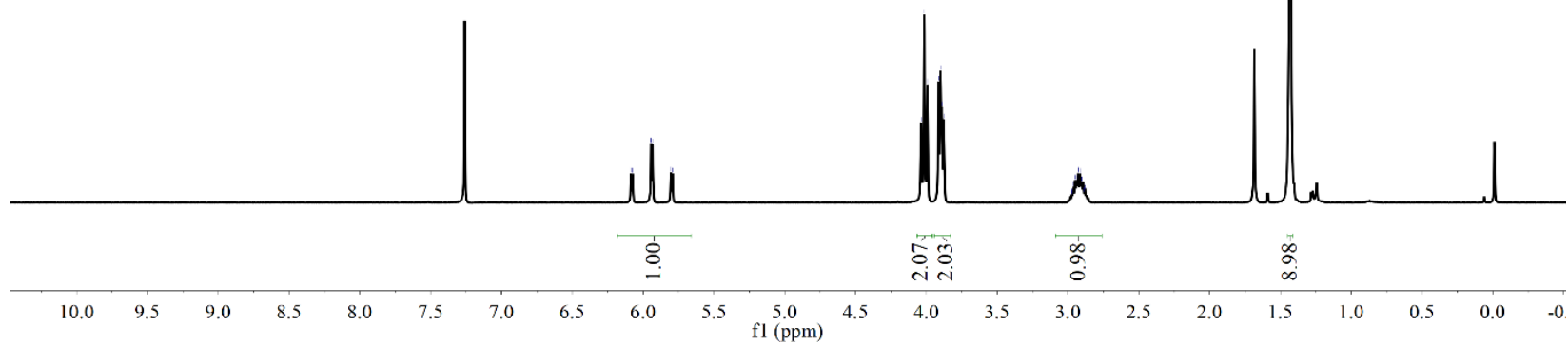

$\overbrace{\substack{N \\ \text { Boc }}}^{\text {F- }}$
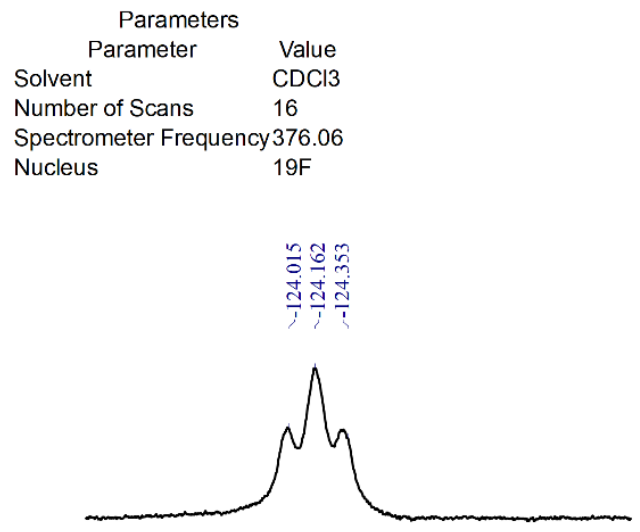

$\begin{array}{lllllllll} & 123.0 & -123.4 & -123.8 & -124.2 & -124.6 & -125.0 & -125.4 & -125.8\end{array}$

$$
\text { fl }(\mathrm{ppm})
$$

$\begin{array}{lllllllllllllllllllllllllll}30 & 20 & 10 & 0 & -10 & -20 & -30 & -40 & -50 & -60 & -70 & -80 & -90 & -100 & -110 & -120 & -130 & -140 & -150 & -160 & -170 & -180 & -190 & -200\end{array}$ 

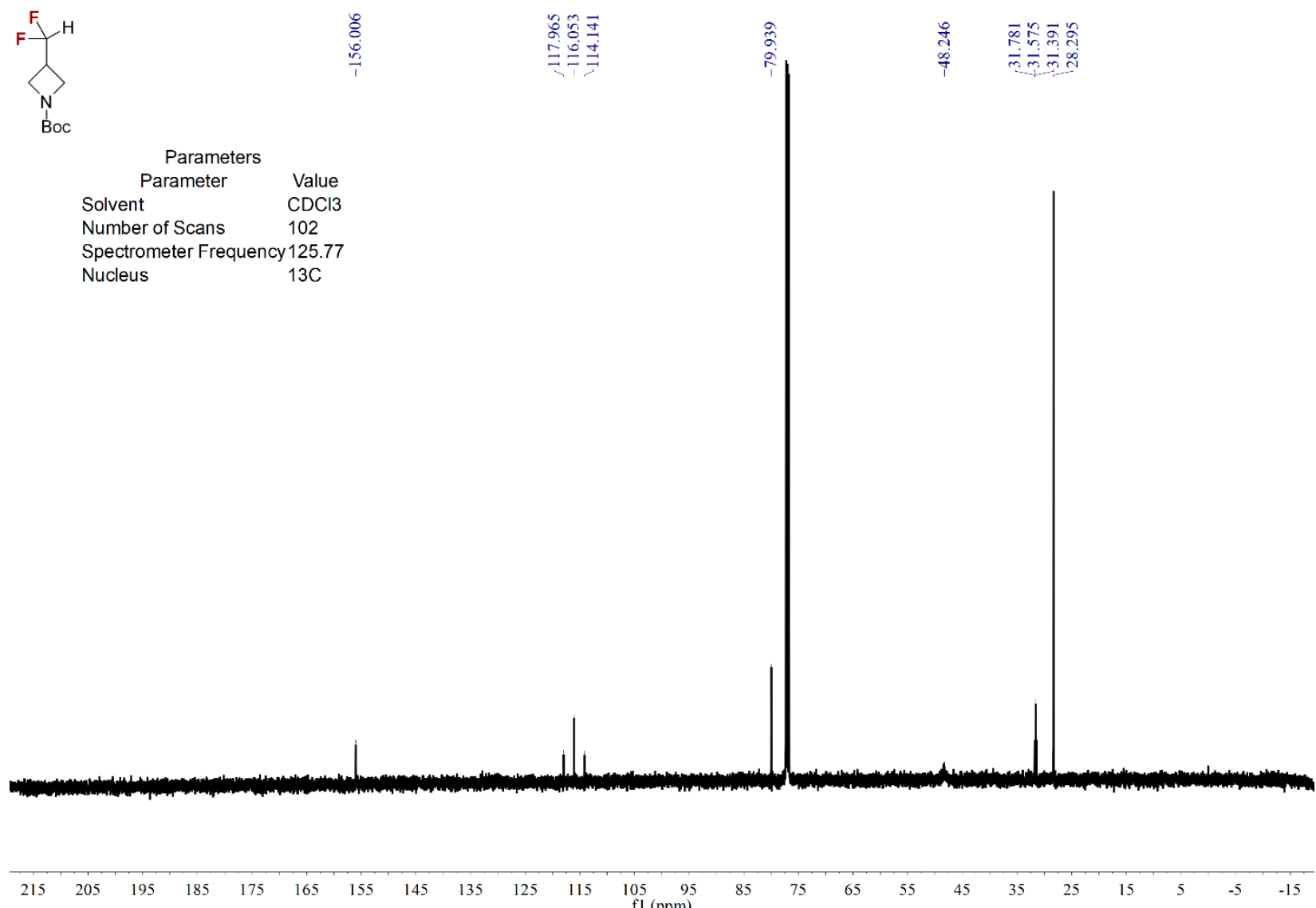

Tert-butyl (1,1-difluoro-3-phenylpropan-2-yl) carbamate (5c)
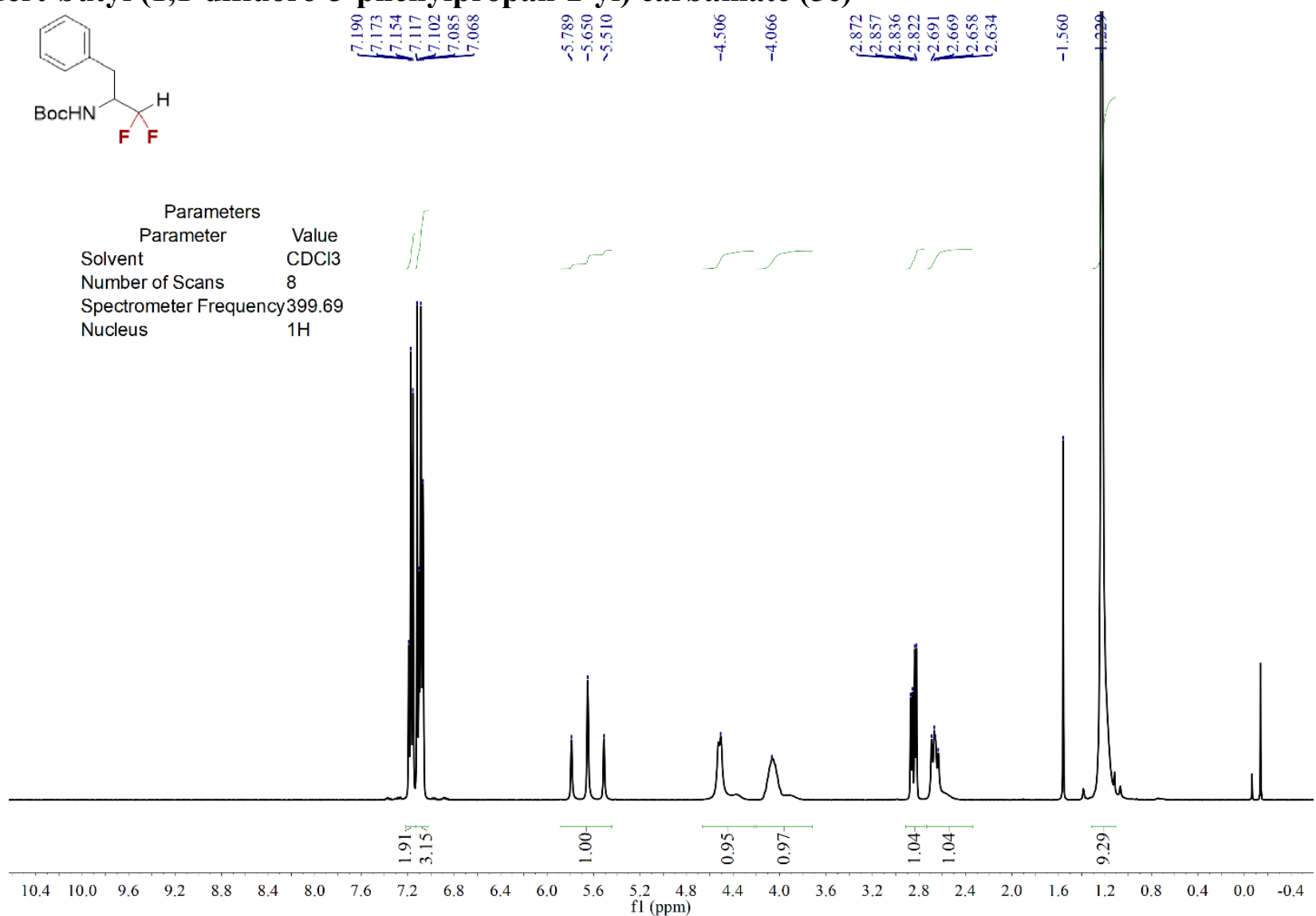


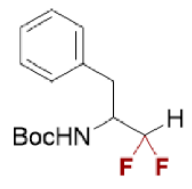

Parameters

\begin{tabular}{ll}
\multicolumn{2}{c}{ Parameters } \\
Parameter & Value \\
Solvent & $\mathrm{CDCl} 3$ \\
Number of Scans & 16 \\
Spectrometer Frequency 376.05 \\
Nucleus & $19 \mathrm{~F}$
\end{tabular}

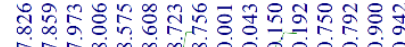

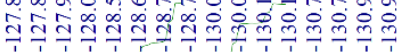

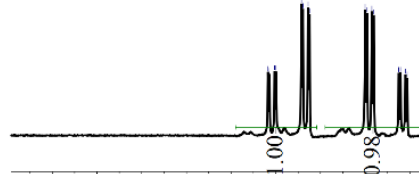

$-123-124-125-126-127-128-129-130-131-132-133-134$ fl (ppm)

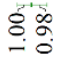

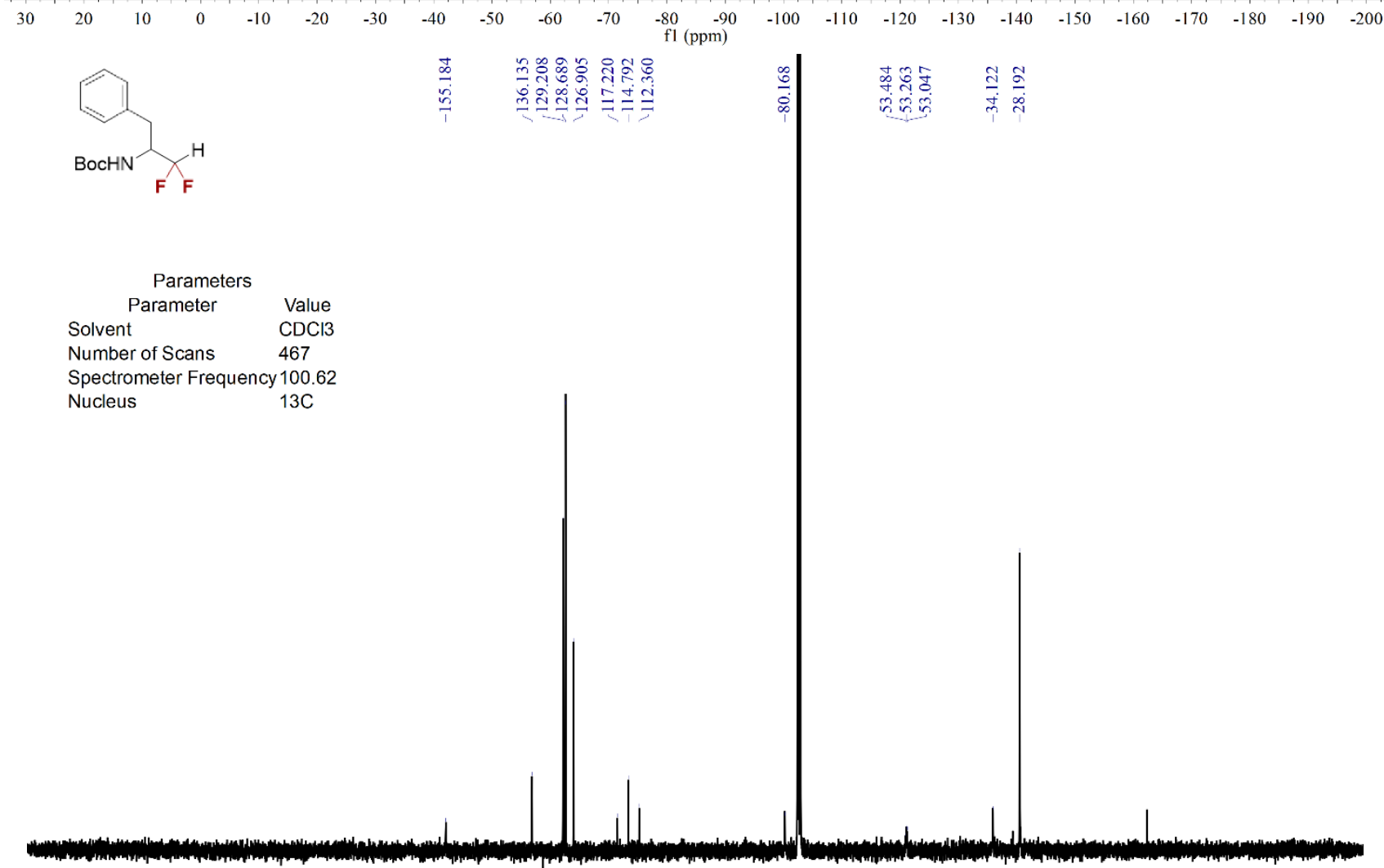
$\begin{array}{lllllllllllllllllllllllllllllllllll}245 & 235 & 225 & 215 & 205 & 195 & 185 & 175 & 165 & 155 & 145 & 135 & 125 & 115 & \begin{array}{l}105 \\ \mathrm{fl}(\mathrm{ppm})\end{array} & 85 & 75 & 65 & 55 & 45 & 35 & 25 & 15 & 5 & -5 & -15 & -25 & -35 & -45\end{array}$ 


\section{2-((5,5-Difluoro-4,4-dimethylpentyl)oxy)-1,4-dimethyl-benzene (5d)}

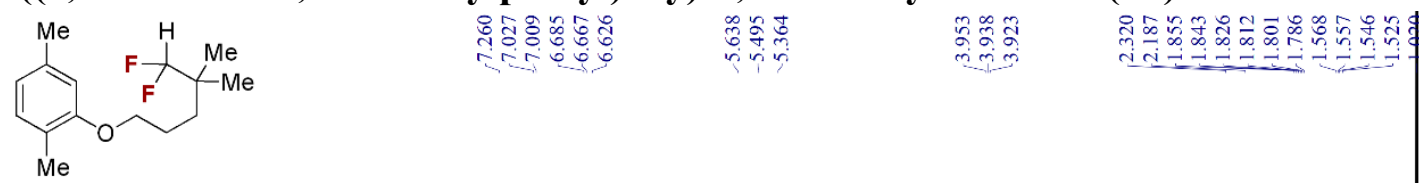

Parameters

Parameter Value
Solvent

Number of Scans 8

Spectrometer Frequency 399.70

Nucleus

$1 \mathrm{H}$
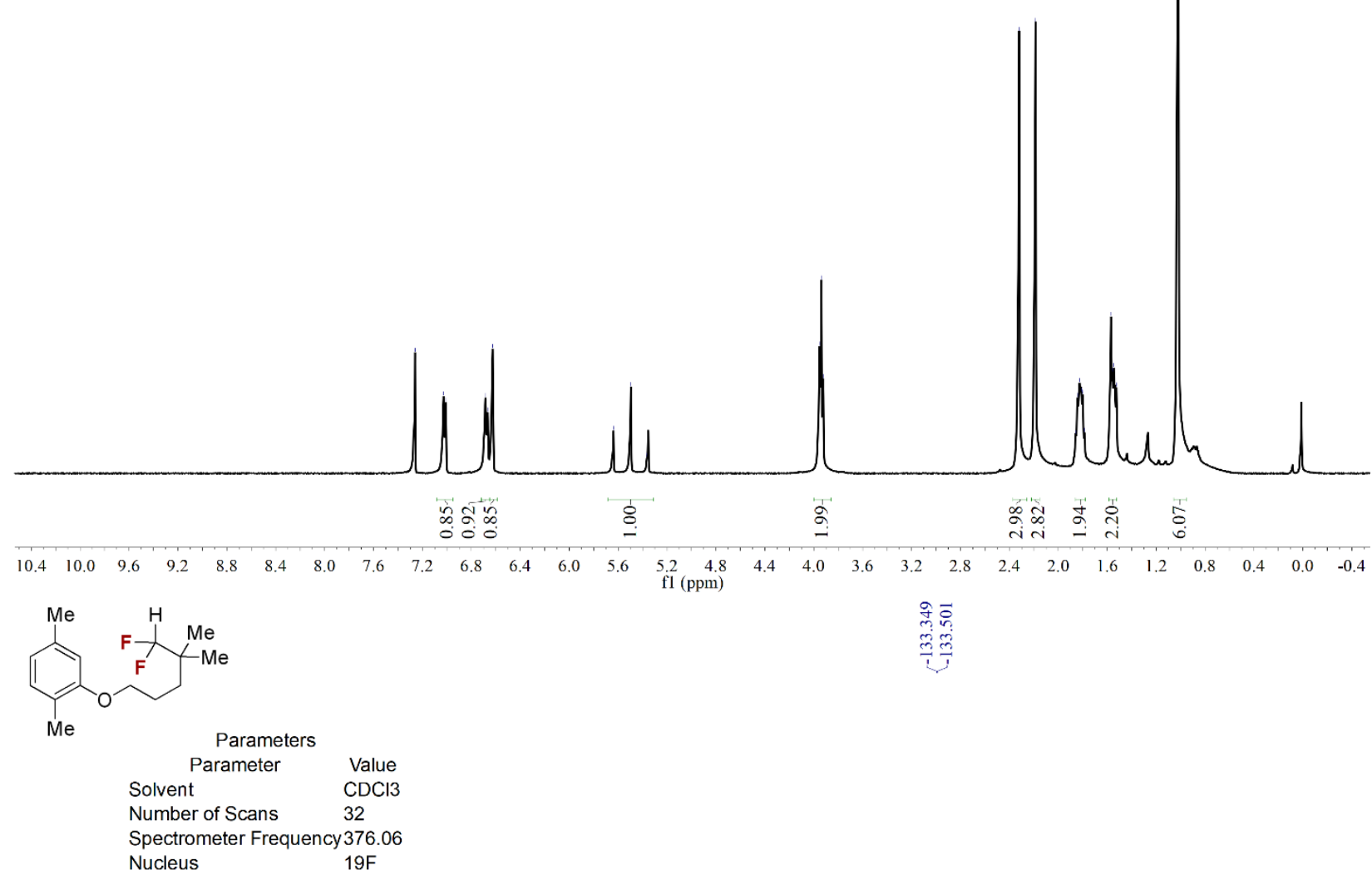

ले $\vec{\circ}$

$m \frac{m}{i}$

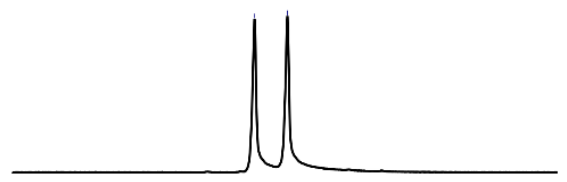

守痛

mim

pectrometer Frequency 376.06

$-132.3-132.6-132.9-133.2-133.5$
f1 (ppm)

$\begin{array}{llllllllllllll}45 & 35 & 25 & 15 & 5 & -5 & -15 & -25 & -35 & -45 & -55 & -65 & -75 & \begin{array}{l}-85 \\ \mathrm{fl}(\mathrm{ppm})\end{array}\end{array}$ 


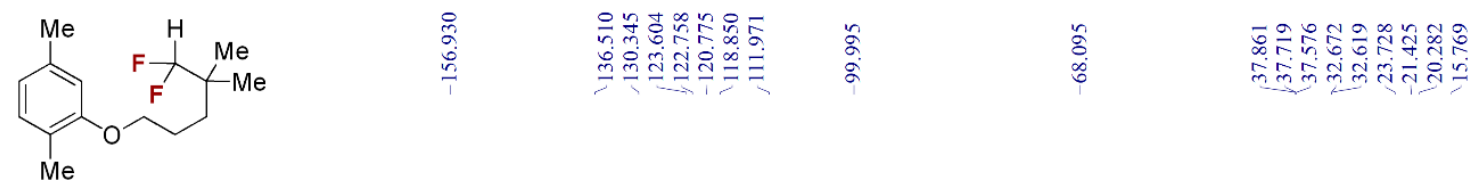

\begin{tabular}{|c|c|}
\hline \multicolumn{2}{|c|}{ Parameters } \\
\hline Parameter & Value \\
\hline Solvent & $\mathrm{CDCl} 3$ \\
\hline Number of Scans & 59 \\
\hline spectrometer Freq & \\
\hline ucleus & 13 \\
\hline
\end{tabular}

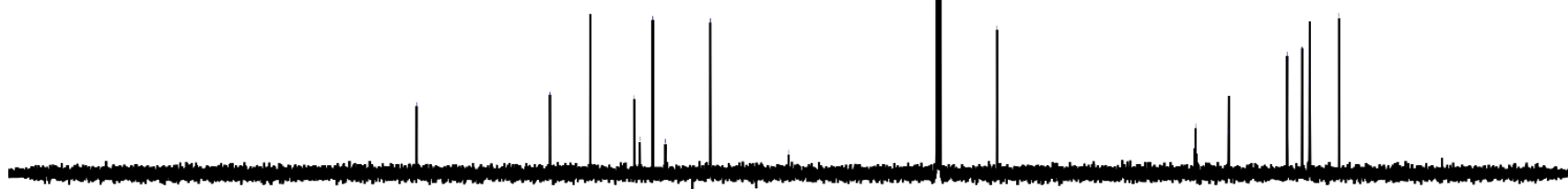

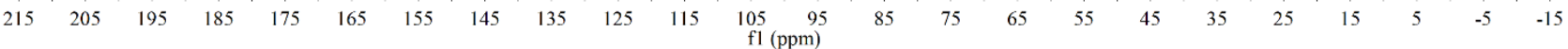

tert-Butyl 4-(1,1-difluoro-2-oxo-2-(p-tolyloxy) ethyl) piperidine-1-carboxylate (6a)

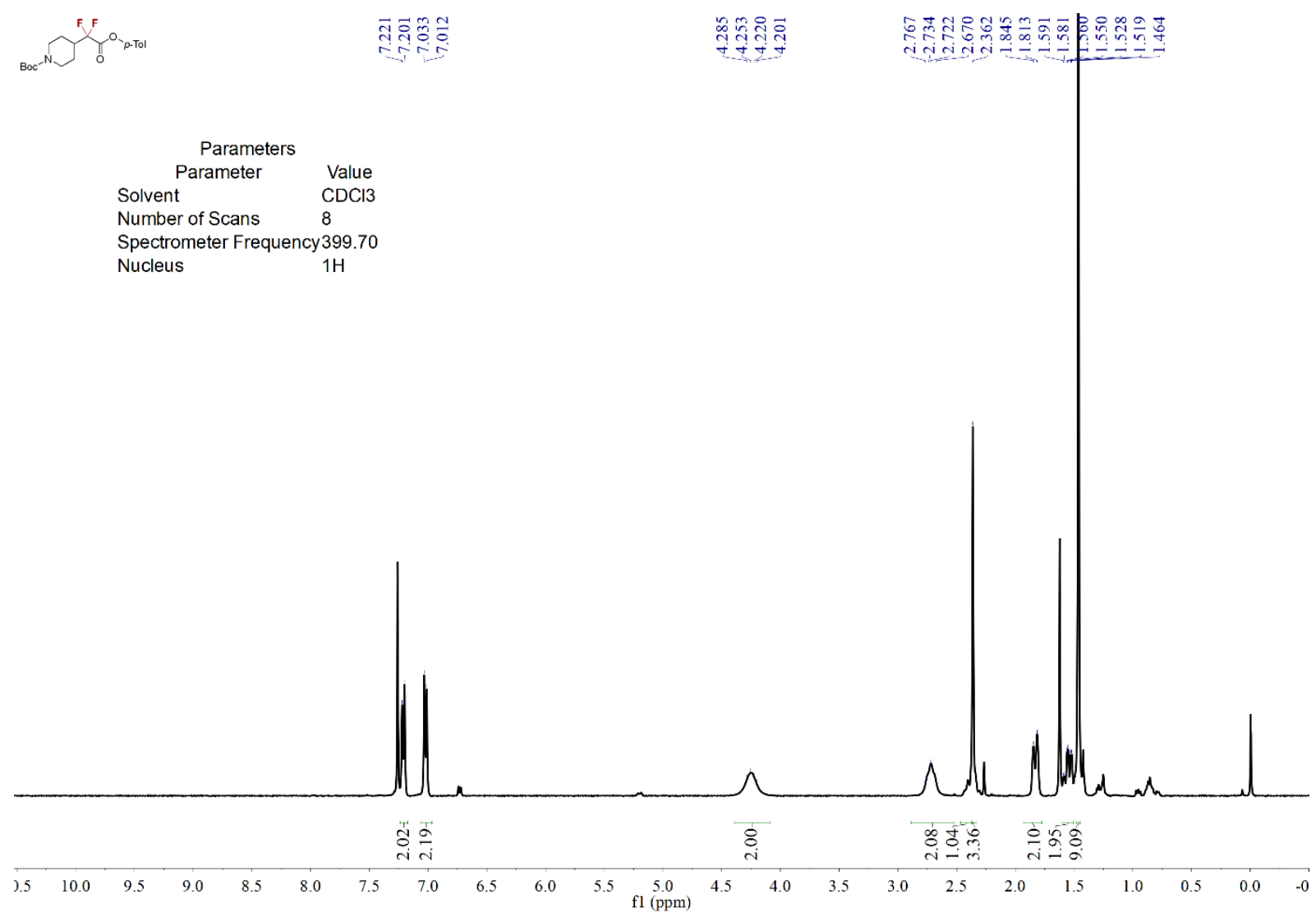


$\overbrace{B o c}^{-N} \int_{0}^{O-D-T}$

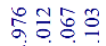

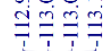

Parameters

Parameter Value

Solvent $\quad \mathrm{CDCl} 3$

Number of Scans

32

Spectrometer Frequency 376.06

Nucleus

$19 \mathrm{~F}$

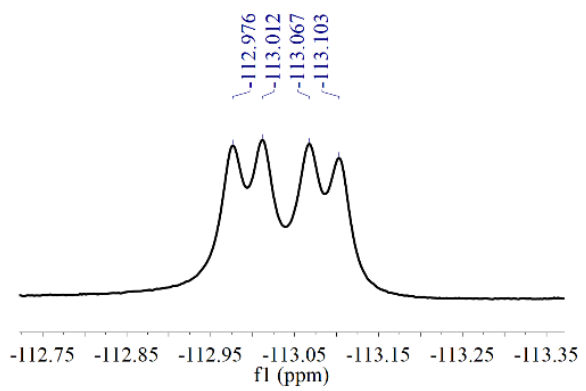

$\begin{array}{llllllllllllllllllllllllllllllllllllll}45 & 35 & 25 & 15 & 5 & -5 & -15 & -25 & -35 & -45 & -55 & -65 & -75 & -85 & -95 & -105 & -115 & -125 & -135 & -145 & -155 & -165 & -175 & -185 & -195 & -205 & -215\end{array}$

Parameters
Parameter
Value
Solvent
Number of Scans
Spectrometer Frequency 125.77
Nucleus
CDCl3

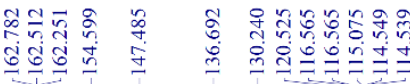

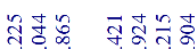

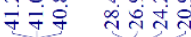

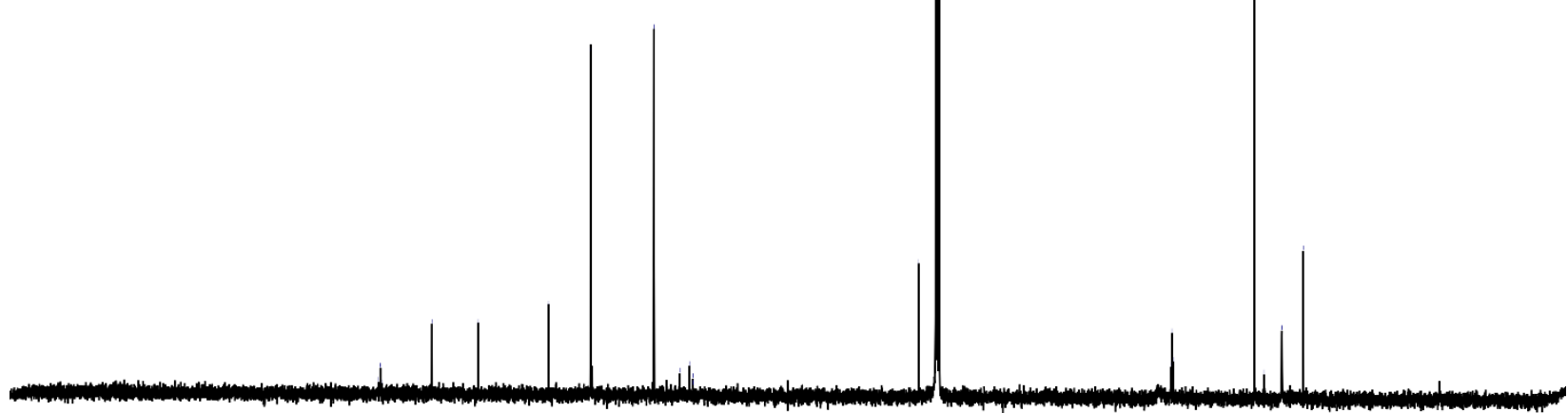

$\begin{array}{lllllllllllll}215 & 205 & 195 & 185 & 175 & 165 & 155 & 145 & 135 & 125 & 115 & 105 & 95\end{array}$

$85 \quad 75 \quad 65 \quad 55$

45

25 
p-Tolyl 3-((tert-butoxycarbonyl)amino)-2,2-difluoro-4-phenylbutanoate (6b)

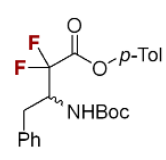

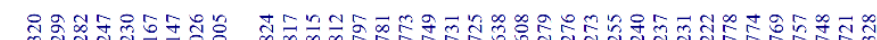

my.

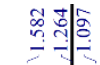

Parameters

Parameter

Solvent

Value

Spectrometer Frequency399.69

Nucleus

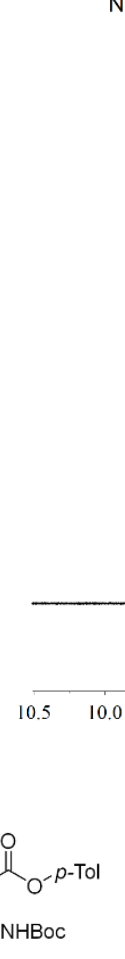

$1 \mathrm{H}$

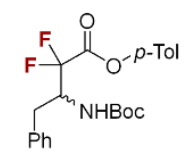

\begin{tabular}{ll}
\multicolumn{1}{c}{ Parameters } \\
Parameter & Value \\
Solvent & CDCl3 \\
Number of Scans & 16 \\
Spectrometer Frequency 376.05 \\
Nucleus & $19 \mathrm{~F}$
\end{tabular}

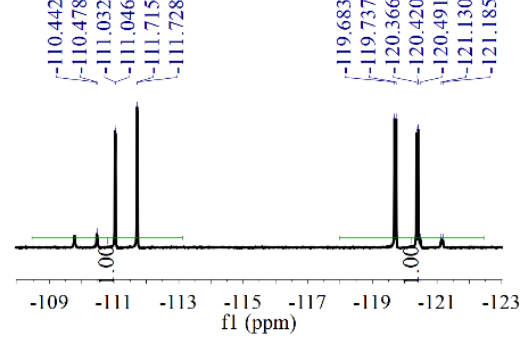

8

\begin{tabular}{llllllllllllllllllllllllllllll}
\hline 30 & 20 & 10 & 0 & -10 & -20 & -30 & -40 & -50 & -60 & -70 & -80 & -90 & -100 & -110 & -120 & -130 & -140 & -150 & -160 & -170 & -180 & -190 & -200
\end{tabular} 


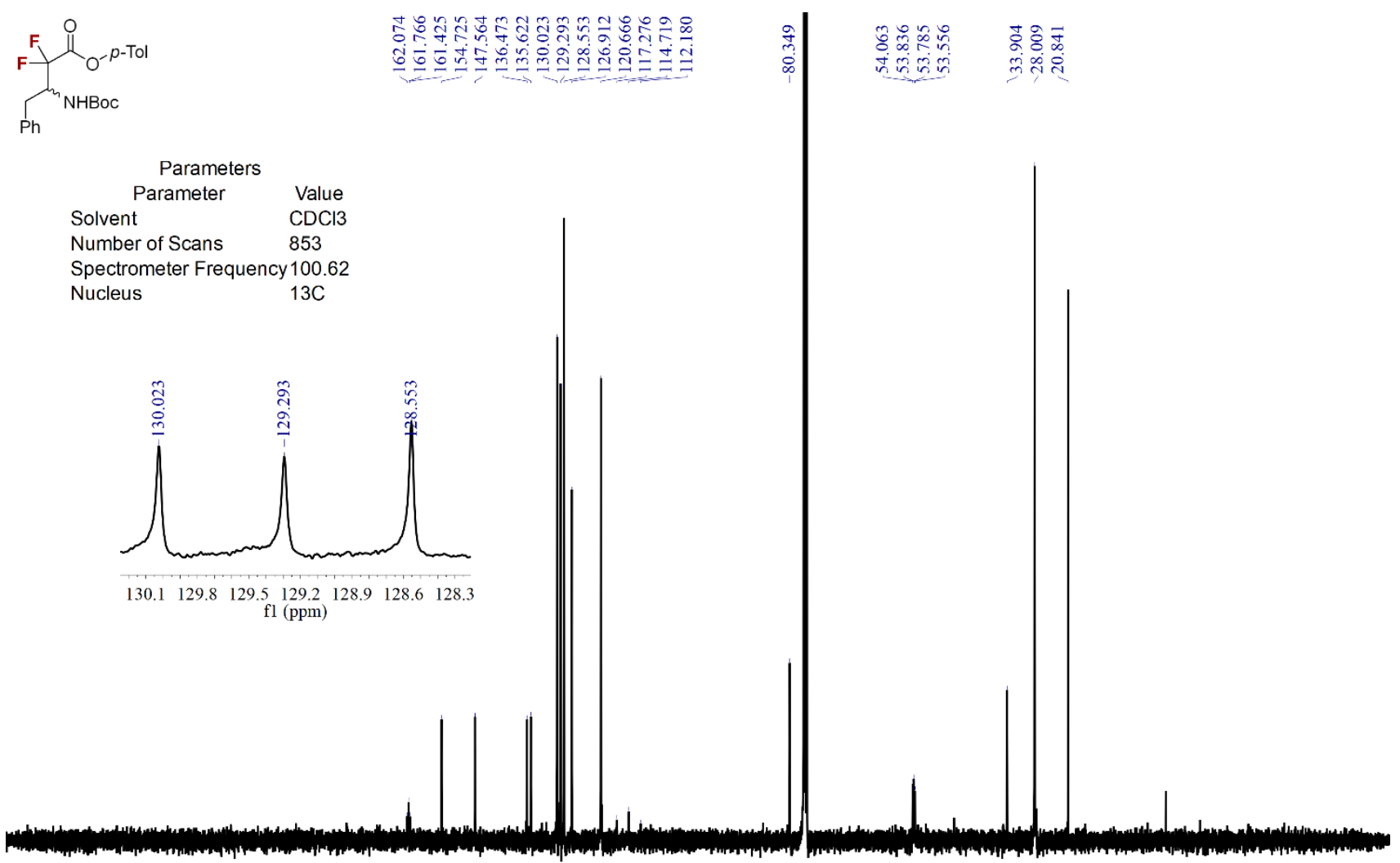

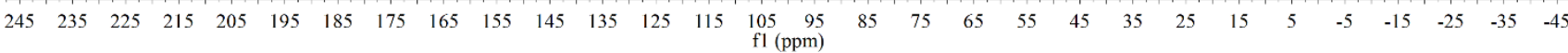
tert-Butyl 4-(1,1-difluoro-2-hydroxy-2-(p-tolyl)ethyl)piperidine-1-carboxylate (7).

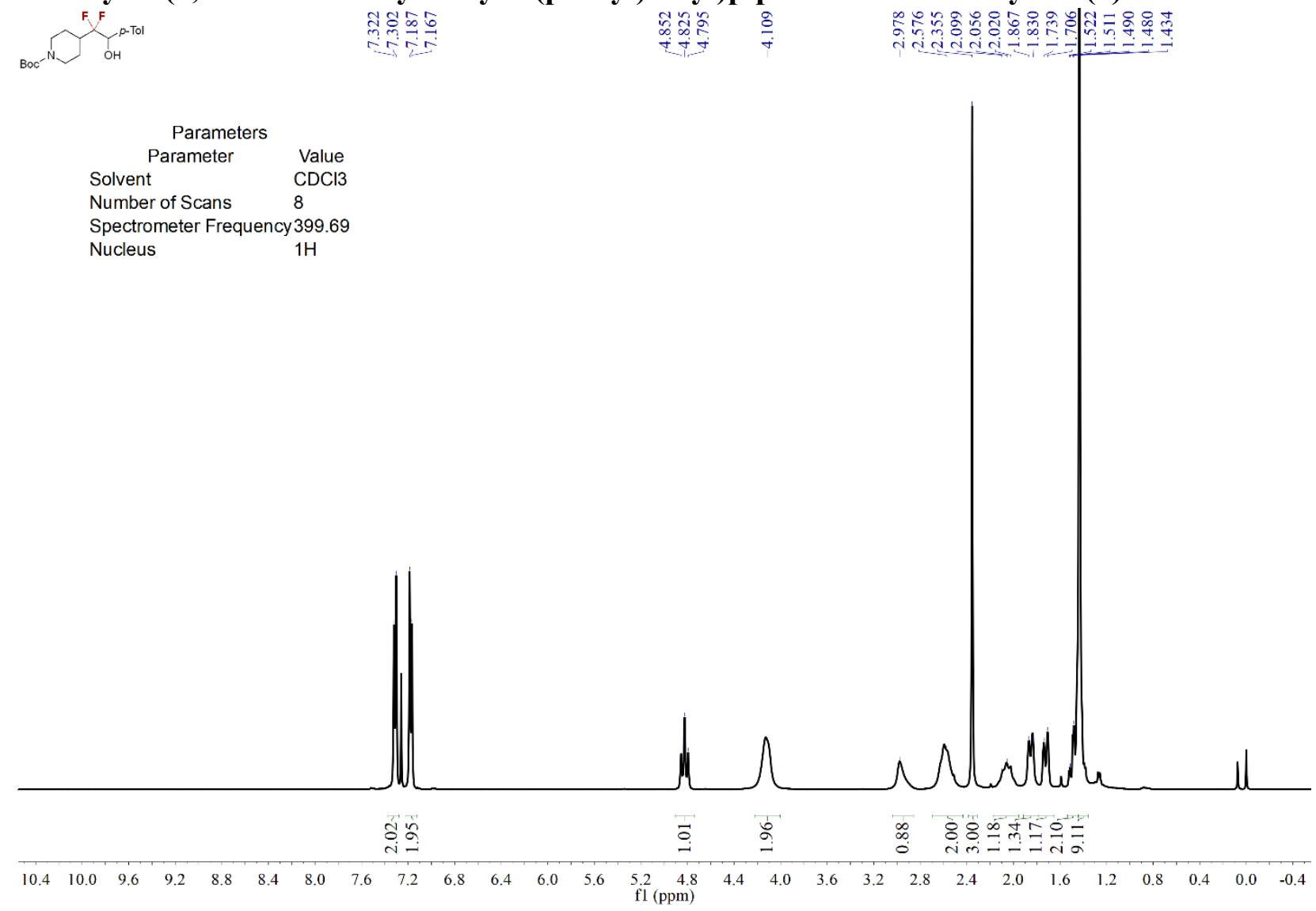


$\overbrace{B O C}^{-N} \int_{O H}^{p-T o l}$

\begin{tabular}{cc}
\multicolumn{1}{c}{ Parameters } \\
Parameter & Value \\
Solvent & CDCl3 \\
Number of Scans & 16 \\
Spectrometer Frequency 376.05 \\
Nucleus & $19 \mathrm{~F}$
\end{tabular}
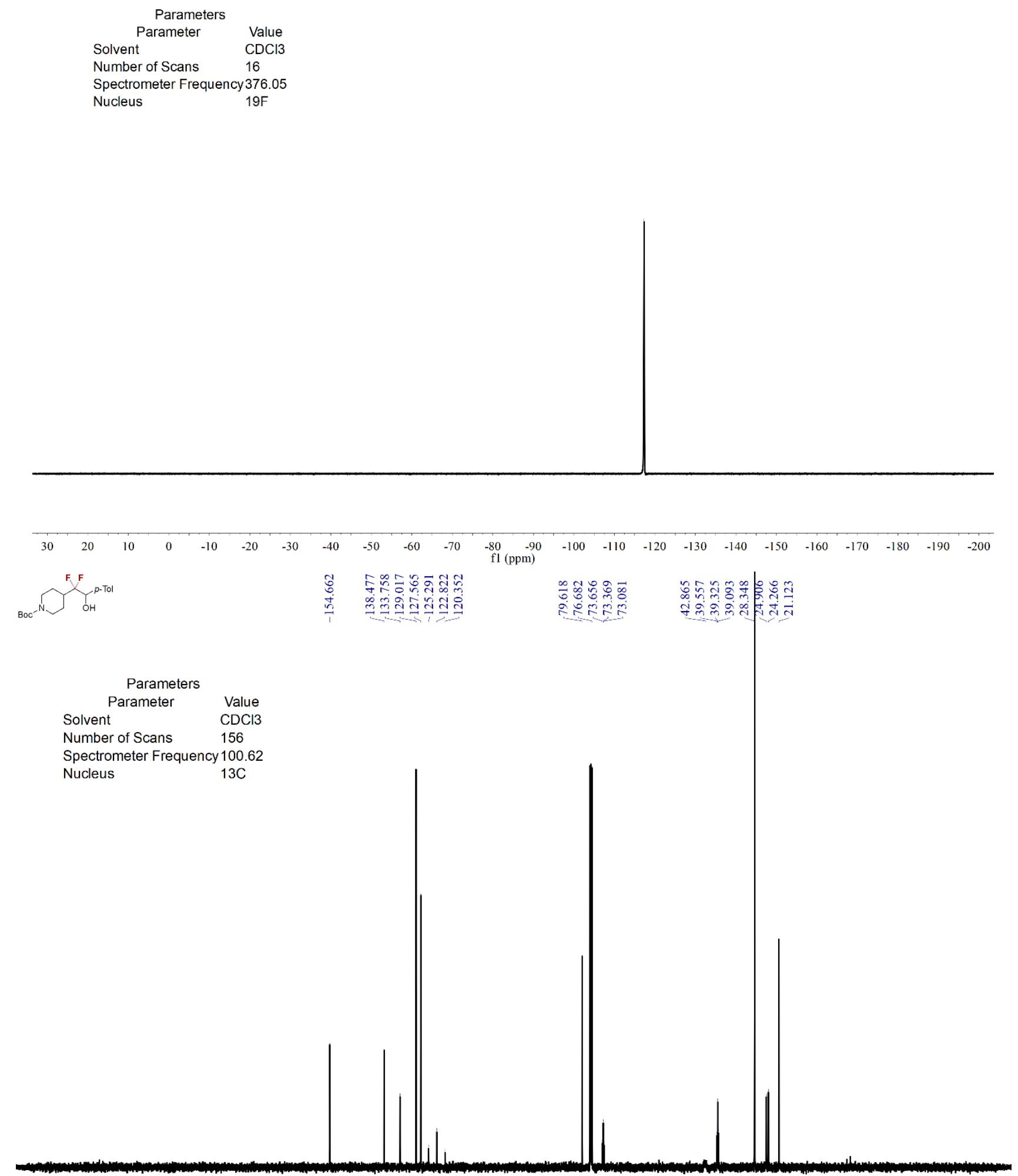

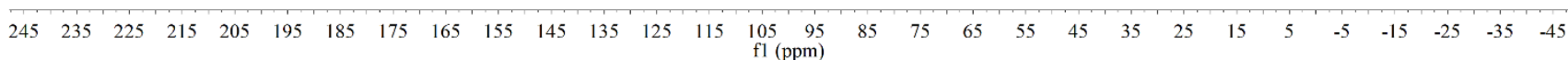

